\title{
The Calderón-Zygmund theory for elliptic problems with measure data
}

\author{
GiUseppe Mingione
}

To the memory of Vic Mizel, mathematician and gentleman

\begin{abstract}
We consider non-linear elliptic equations having a measure in the right-hand side, of the type $\operatorname{div} a(x, D u)=\mu$, and prove differentiability and integrability results for solutions. New estimates in Marcinkiewicz spaces are also given, and the impact of the measure datum density properties on the regularity of solutions is analyzed in order to build a suitable Calderón-Zygmund theory for the problem. All the regularity results presented in this paper are provided together with explicit local a priori estimates.
\end{abstract}

Mathematics Subject Classification (2000): 35J60 (primary); 35J70 (secondary).

\section{Contents}

1 Introduction and results . . . . . . . . . . . . . . . . . 195

2 Preliminaries, function spaces . . . . . . . . . . . . . 205

3 Regularity for homogeneous problems . . . . . . . . . . . . 213

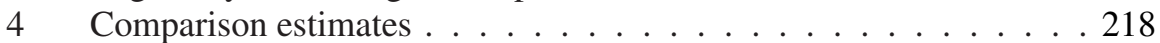

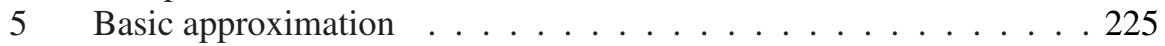

6 General measures . . . . . . . . . . . . . . . . 226

7 The capacitary case . . . . . . . . . . . . . . . 236

8 Morrey estimates . . . . . . . . . . . . . . . . . . . . 239

9 Marcinkiewicz estimates ... . . . . . . . . . . . . . 243

10 The super-capacitary case . . . . . . . . . . . . . 251

11 Sharpness, comparisons, extensions . . . . . . . . . . 255

References . . . . . . . . . . . . . . . . . 258

\section{Introduction and results}

Let us consider the following Dirichlet problem:

$$
\begin{cases}-\operatorname{div} a(x, D u)=\mu & \text { in } \Omega \\ u=0 & \text { on } \partial \Omega .\end{cases}
$$

Received January 12, 2007; accepted March 30, 2007. 
Here we assume that $\Omega \subset \mathbb{R}^{n}$ is a bounded domain, $\mu$ is a signed Radon measure with finite total variation $|\mu|(\Omega)<\infty$, and $a: \Omega \times \mathbb{R}^{n} \rightarrow \mathbb{R}^{n}$ is a Carathèodory vector field satisfying the following standard monotonicity and Lipschitz assumptions:

$$
\left\{\begin{array}{l}
v\left(s^{2}+\left|z_{1}\right|^{2}+\left|z_{2}\right|^{2}\right)^{\frac{p-2}{2}}\left|z_{2}-z_{1}\right|^{2} \leq\left\langle a\left(x, z_{2}\right)-a\left(x, z_{1}\right), z_{2}-z_{1}\right\rangle \\
\left|a\left(x, z_{2}\right)-a\left(x, z_{1}\right)\right| \leq L\left(s^{2}+\left|z_{1}\right|^{2}+\left|z_{2}\right|^{2}\right)^{\frac{p-2}{2}}\left|z_{2}-z_{1}\right| \\
|a(x, 0)| \leq L s^{p-1}
\end{array}\right.
$$

for every $z_{1}, z_{2} \in \mathbb{R}^{n}, x \in \Omega$. Here, and in the rest of the paper, when referring to the structural properties of $a$, and in particular to (1.2), we shall always assume

$$
p \geq 2, \quad n \geq 2, \quad 0<v \leq L, \quad s \geq 0 .
$$

The measure $\mu$ will be considered as defined on the whole $\mathbb{R}^{n}$ by simply letting $|\mu|\left(\mathbb{R}^{n} \backslash \Omega\right)=0$. At certain stages, we shall also require the following Lipschitz continuity assumption on the map $x \mapsto a(x, z)$ :

$$
\left|a(x, z)-a\left(x_{0}, z\right)\right| \leq L\left|x-x_{0}\right|\left(s^{2}+|z|^{2}\right)^{\frac{p-1}{2}}, \quad \forall x, x_{0} \in \Omega, z \in \mathbb{R}^{n} .
$$

Assumptions (1.2) are modeled on the basic example

$$
-\operatorname{div}\left[c(x)\left(s^{2}+|D u|^{2}\right)^{\frac{p-2}{2}} D u\right]=\mu, \quad v \leq c(x) \leq L,
$$

which is indeed covered here. When $s=0$ and $c(x) \equiv 1$ we have the familiar $p$-Laplacean operator on the left-hand side

$$
-\triangle_{p} u=-\operatorname{div}\left(|D u|^{p-2} D u\right)=\mu .
$$

For the problem (1.1) in the rest of the paper we shall adopt the following distributional-like notion of solution, compare with [8] for instance.

Definition 1.1. A solution $u$ to the problem (1.1) under assumptions (1.2), is a function $u \in W_{0}^{1,1}(\Omega)$ such that $a(x, D u) \in L^{1}\left(\Omega, \mathbb{R}^{n}\right)$ and

$$
\int_{\Omega} a(x, D u) D \varphi d x=\int_{\Omega} \varphi d \mu, \quad \text { for every } \varphi \in C_{c}^{\infty}(\Omega) .
$$

The existence of such a solution is usually obtained combining a priori estimates with a suitable approximation scheme [8,20,27], see also Section 5 below. The same approach is followed here and therefore in the rest of the paper when talking about regularity we shall refer to that of Solutions Obtained as Limits of Approximations (SOLA) [7,20], and we shall actually simultaneously obtain existence and regularity results. Here we just want to recall that uniqueness of solutions to (1.1) 
in the sense of Definition 1.1 generally fails [67], and a main open problem of the theory is identifying a suitable functional class where a unique solution can be defined and found. In this respect many possible definitions have been proposed, and technically demanding attempts have been made: for this we refer for instance to $[6,10,45,63]$, and to the references therein. Nevertheless, a general uniqueness theory is still missing except for $p=2$ or $p=n[7,27,35]$; in particular we refer to the paper [21] for a rather comperhensive discussion about the uniqueness problem, and measure data problems in general. We shall not discuss uniqueness problems any further, our aims here being quite different: we are mainly interested in a priori regularity estimates. For the same reason, we shall confine ourselves to distributional solutions as defined in (1.1), while the results we are going to propose could be approached also for other notions of solutions: entropy ones, for instance.

The study of problem (1.1) began with the fundamental work of Littman \& Stampacchia \& Weinberger $[54,68]$, who defined solutions in a duality sense in the case of linear equations with measurable coefficients: $a_{i}(x, z) \equiv \tilde{a}_{i j}(x) z_{j}$. When referring to Definition 1.1, the existence theory for the general quasi linear LerayLions type operators in $(1.1)_{1}$ has been established in the by now classical paper of Boccardo \& Gallouët [8], who proved the existence of a solution $u$ to problem (1.1) such that

$$
D u \in L^{q}\left(\Omega, \mathbb{R}^{n}\right), \quad \text { for every } q<b \text { when } p \leq n,
$$

where

$$
b:=\frac{n(p-1)}{n-1}
$$

Dolzmann \& Hungerbühler \& Müller were able to prove the same result for a large class of systems including the $p$-Laplacean one [26,27]. Inclusion (1.8) is optimal in the scale of Lebesgue spaces, see Section 11.1, as $D u \notin L^{b}$ in general. Anyway (1.8) can be sharpened using Marcinkiewicz spaces [6,27], see (2.17) below, since

$$
D u \in \mathcal{M}^{b}\left(\Omega, \mathbb{R}^{n}\right) .
$$

When $p>n$ instead, $\mu$ belongs to $W^{-1, p^{\prime}}$, that is the dual of $W^{1, p}$, and the existence of a unique solution in the natural space $W_{0}^{1, p}(\Omega)$ follows by standard duality methods [53]. Related regularity results for the equation (1.6) with a nonnegative measure $\mu$ were given by Lindqvist [51], in connection to the notion of $p$-superharmonic functions; see also [37] for a fairly comprehensive treatment of this subject. Related estimates and problems, using various techniques, are in $[22,32,37,42,70]$.

\subsection{General measures}

Up to now, regularity results in $L^{q}$ spaces of the type in (1.8)-(1.10) are the only ones available in the literature. One of the aims of this paper is to give the first higher regularity results for the gradient of solutions, in particular estimating the oscillations of the gradient rather than its size. Let us focus for simplicity on the 
case $p=2$, looking at (1.8) from a different viewpoint, considering $\Delta u=f$. In this case the standard Calderón-Zygmund theory [33] asserts

$$
f \in L^{1+\varepsilon} \Longrightarrow D u \in W^{1,1+\varepsilon} \quad \text { for every } \varepsilon>0 .
$$

Using Sobolev's embedding theorem we have in particular $D u \in L^{n /(n-1)}$, that is, the limit case of (1.8). This does not hold when $\varepsilon=0$, since the inclusion $D u \in W^{1,1}$ generally fails. So, one could interpret (1.8) as the trace of a potentially existent Calderón-Zygmund theory below the limit case $W^{1,1}$. Indeed we have:

Theorem 1.2 (of Calderón-Zygmund type). Under the assumptions (1.2) and (1.4) with $p \leq n$, there exists a solution $u \in W_{0}^{1,1}(\Omega)$ to the problem (1.1) such that

$$
D u \in W_{\mathrm{loc}}^{\frac{1-\varepsilon}{p-1}, p-1}\left(\Omega, \mathbb{R}^{n}\right),
$$

for every $\varepsilon \in(0,1)$, and in particular

$$
D u \in W_{\text {loc }}^{1-\varepsilon, 1}\left(\Omega, \mathbb{R}^{n}\right), \quad \text { when } \quad p=2 .
$$

More in general

$$
D u \in W_{\text {loc }}^{\frac{\sigma(q)-\varepsilon}{q}, q}\left(\Omega, \mathbb{R}^{n}\right),
$$

for every $\varepsilon \in(0, \sigma(q))$, where

$$
p-1 \leq q<\frac{n(p-1)}{n-1}=b, \quad \sigma(q):=n-\frac{q(n-1)}{p-1}=n(1-q / b),
$$

and $b$ is in (1.9).

In other words, in (1.13) we "almost have" second derivatives of $u$; see anyway (1.24) below and comments after (1.18). Explicit local estimates are actually available:

Theorem 1.3 (Calderón-Zygmund estimates I). Under the assumptions and notations of Theorem 1.2, let $q \in[p-1, b)$ and $\sigma \in(0, \sigma(q))$. There exists a constant $c \equiv c(n, p, L / \nu, q, \sigma)$ such that for every ball $B_{R} \subset \subset \Omega$ of radius $R>0$ it holds

$$
\begin{aligned}
\int_{B_{R / 2}} \int_{B_{R / 2}} \frac{|D u(x)-D u(y)|^{q}}{|x-y|^{n+\sigma}} d x d y \leq & \frac{c}{R^{\sigma}} \int_{B_{R}}\left(|D u|^{q}+s^{q}\right) d x \\
& +c R^{\sigma(q)-\sigma}\left[|\mu|\left(\overline{B_{R}}\right)\right]^{\frac{q}{p-1}} .
\end{aligned}
$$

Moreover, for any open subset $\Omega^{\prime} \subset \subset \Omega$ the local estimate

$$
\int_{\Omega}|D u|^{q} d x+\int_{\Omega^{\prime}} \int_{\Omega^{\prime}} \frac{|D u(x)-D u(y)|^{q}}{|x-y|^{n+\sigma}} d x d y \leq c[|\mu|(\Omega)]^{\frac{q}{p-1}}+c s^{q}|\Omega|
$$

holds, where $c \equiv c\left(n, p, L / \nu, q, \sigma, \operatorname{dist}\left(\Omega^{\prime}, \partial \Omega\right), \Omega\right)$. 
Therefore, it is possible to establish an optimal Calderón-Zygmund theory for non-linear elliptic problems with measure data, provided the right Sobolev spaces are considered: that is, the fractional ones; see the definition in (2.9). Fractional Sobolev spaces are an essential tool in modern analysis in that they provide the natural intermediate scale to state optimal regularity results, and to show the persistence of certain assertions up to the so called "limit cases". Inclusion (1.13) is an instance of this situation, and the comparison between (1.13) and (1.11) tells us that Calderón-Zygmund theory does not have $W^{1,1}$ as an endpoint, but it continues below $W^{1,1}$. Inclusions (1.12)-(1.14) are sharp for every choice of the couple $(q, \sigma(q))$ in $(1.15)$ as $D u \notin W_{\text {loc }}^{\sigma(q) / q, q}$ in general; note that $(p-1, \sigma(p-1))=(p-1,1)$. On the converse, inclusion (1.14) admits (1.8) as a corollary, at least in a local fashion; see Section 11.1. When $p \neq 2$, as $\varepsilon \searrow 0$ we do not approach an integer fractional differentiability exponent in (1.12), as for (1.13), but only $1 /(p-1)$. This is not a surprise: even for the model case

$$
\triangle_{p} u=0
$$

the existence of second derivatives of $W^{1, p}$-solutions is not clear due to the degeneracy of the problem, while fractional derivatives naturally appear [59]

$$
u \in W^{1, p} \Longrightarrow D u \in W^{2 / p, p} \text {. }
$$

On the other hand, a classical result going back to K. Uhlenbeck [72] asserts that although $D u$ may be not differentiable for (1.18), certain natural non-linear expressions of the gradient still are (in T. Iwaniec's words [28]). Indeed, defining

$$
V(D u):=\left(s^{2}+|D u|^{2}\right)^{\frac{p-2}{4}} D u,
$$

then under the assumptions (1.2) we have that $V(D u) \in W_{\text {loc }}^{1,2}\left(\Omega, \mathbb{R}^{n}\right)$ for any $W^{1, p}$-solution to

$$
\operatorname{div} a(D u)=0 ;
$$

see Lemma 3.2 below, and under stronger assumptions also [36, 56, 57], [34, Chapter 8], and [52, Chapter 4]. See also Section 11.2 for more comments on the fact that passing to $V(D u)$ allows for a gain in differentiability. Observe that the main essence here is that the product between the differentiability and the integrability indexes of the fractional spaces involved for $D u$ and $V(D u)$, respectively, is invariant

$$
\frac{2}{p} \cdot p=1 \cdot 2 \text {. }
$$

This phenomenon extends to measure data problems as well:

Theorem 1.4 (Non-linear Calderón-Zygmund estimates). Under the assumptions (1.2) and (1.4) with $p \in(2, n]$, let $u \in W_{0}^{1, q}(\Omega)$ be the solution to (1.1) found in Theorem 1.2. Then

$$
V(D u) \in W_{\mathrm{loc}}^{\frac{p}{2(p-1)}-\varepsilon, \frac{2(p-1)}{p}}\left(\Omega, \mathbb{R}^{n}\right), \quad \text { for every } \varepsilon \in(0,1) .
$$


Moreover, for any open subset $\Omega^{\prime} \subset \subset \Omega$, we have

$$
\int_{\Omega^{\prime}} \int_{\Omega^{\prime}} \frac{|V(D u(x))-V(D u(y))|^{\frac{2(p-1)}{p}}}{|x-y|^{n+1-\varepsilon}} d x d y \leq \tilde{c}|\mu|(\Omega)+\tilde{c} s^{p-1}|\Omega|,
$$

where $\tilde{c} \equiv \tilde{c}\left(n, p, L / \nu, \varepsilon, \operatorname{dist}\left(\Omega^{\prime}, \partial \Omega\right), \Omega\right)$.

When the problem is non-degenerate, that is $s>0$ in (1.3), something more can be proved: $W^{1, p}$-solutions to (1.20) belong to $W_{\text {loc }}^{2,2}$. The following corollary of Theorem 1.4 contains the analogue in the measure data case.

Corollary 1.5 (The non-degenerate case). Under the assumptions (1.2) and (1.4) with $p \in(2, n]$, let $u \in W_{0}^{1, q}(\Omega)$ be the solution to (1.1) found in Theorem 1.2, and assume $s>0$. Then

$$
D u \in W_{\text {loc }}^{\frac{p}{2(p-1)}-\varepsilon, \frac{2(p-1)}{p}}\left(\Omega, \mathbb{R}^{n}\right), \quad \text { for every } \varepsilon \in(0,1) .
$$

Moreover estimate (1.23) holds with Du replacing $V(D u)$ provided the constant $\tilde{c}$ is replaced by the new one: $s^{(2-p)(p-1) / p} c(n, p) \tilde{c}$.

Remark 1.6. In Theorems 1.3 and 1.4 , and Corollary 1.5, the constants $c, \tilde{c}$ depending on $q, \varepsilon, \sigma$ blow-up as $q \nearrow b, \varepsilon \searrow 0, \sigma \nearrow \sigma(q)$. Also observe that formally letting $p=2$ in the three previous statements we obtain (1.13).

Combining inclusions (1.13) and (1.22) with Proposition 2.4 below we gain

Corollary 1.7 (BV-type behavior). Let $\Sigma_{u}$ denote the set of non-Lebesgue points of the solution found in Theorem 1.2, in the sense of

$$
\begin{aligned}
& \Sigma_{u}:=\left\{x \in \Omega: \liminf _{\rho \searrow 0} f_{B(x, \rho)}\left|D u(y)-(D u)_{x, \rho}\right| d y>0\right. \\
& \text { or } \left.\quad \limsup _{\rho \searrow 0}\left|(D u)_{x, \rho}\right|=\infty\right\} .
\end{aligned}
$$

Then its Hausdorff dimension $\operatorname{dim}\left(\Sigma_{u}\right)$ satisfies

$$
\operatorname{dim}\left(\Sigma_{u}\right) \leq n-1 .
$$

The same result holds replacing $D u$ by $V(D u)$ in (1.25).

Therefore solutions behave as BV functions [5]. For $p=2$ one can guess this, with some brave heuristics, by looking at $\Delta u=\mu$, "replacing" $\Delta u$ by $D^{2} u$.

Before going on let us observe that the above results are only local, while we are dealing with a Dirichlet problem; this is a precise, simplifying choice of ours. Indeed the techniques presented here are suitable to be carried out up to the boundary under additional regularity assumptions on $\partial \Omega$, say $C^{2}$ for instance, or Lipschitz in some cases, but since they are already delicate and involved, at this stage we prefer to confine ourselves to the local versions of the results, in order to highlight the main new ideas. For the case $p<2$ see also [61]; here the results change. 


\subsection{Diffusive measures}

The sharpness of (1.10) and (1.12)-(1.14) stems from considering counterexamples involving Dirac measures, see again Section 11.1. It is therefore natural to wonder whether things change when considering measures diffusing on sets with higher Hausdorff dimension. A natural way to quantify this, also suggested by a classical result of Frostman, see [3, Theorem 5.1.12], is to consider the following density condition:

$$
|\mu|\left(B_{R}\right) \leq M R^{n-\theta} \quad 0 \leq \theta \leq n, \quad M \geq 0,
$$

satisfied for any ball $B_{R} \subset \mathbb{R}^{n}$ of radius $R$. Assuming (1.27) does not allow $\mu$ to concentrate on sets with Hausdorff dimension less than $n-\theta$, and indeed higher regularity of solutions can be obtained. We have anyway to distinguish two cases.

\subsection{The super-capacitary case}

This is when $\theta \geq p$, making sense only if $p \leq n$. We see that in all the above results the role of the dimension $n$ is actually played by $\theta$ in (1.27); in particular, the critical exponent $b$ appearing in Theorem 1.2 and in (1.8) is replaced by the larger one

$$
m:=\frac{\theta(p-1)}{\theta-1} .
$$

The first improvement is in the integrability properties of $D u$, detectable in two different scales: Marcinkiewicz and Morrey ones, see (2.18) and (2.19).

Theorem 1.8 (Marcinkiewicz-Morrey regularity). Under the assumptions (1.2) with $p \leq n$, and (1.27) with $\theta \geq p$, there exists a solution $u \in W_{0}^{1,1}(\Omega)$ to the problem (1.1) such that

$$
D u \in \mathcal{M}_{\mathrm{loc}}^{m, \theta}\left(\Omega, \mathbb{R}^{n}\right) \subseteq \mathcal{M}_{\mathrm{loc}}^{m}\left(\Omega, \mathbb{R}^{n}\right),
$$

where $m$ is in (1.28). Moreover, for any open subset $\Omega^{\prime} \subset \subset \Omega$ we have

$$
\|D u\|_{\mathcal{M}^{m, \theta}\left(\Omega^{\prime}\right)} \leq c M^{\frac{1}{p-1}}+c s|\Omega|^{\frac{1}{m}},
$$

where $c \equiv c\left(n, p, L / v, \Omega^{\prime}, \Omega\right)$, and $M$ appears in (1.27). In particular, in the limit case $\theta=p$ we have

$$
D u \in \mathcal{M}_{\mathrm{loc}}^{p, p}\left(\Omega, \mathbb{R}^{n}\right) \subseteq \mathcal{M}_{\mathrm{loc}}^{p}\left(\Omega, \mathbb{R}^{n}\right) .
$$

The exponent $m$ is expected to be the best possible in (1.29) for every $p \geq 2$, and it actually is when $p=2$, see Section 11.3: Theorem 1.8 may be also regarded as the non-linear version of a classical result of Adams [2]. As explained below, when $\theta<p$, the solution $u$ is uniquely found in $W_{0}^{1, p}(\Omega)$, so that (1.27) provides 
the natural scale that allows to pass from (1.10), when $\theta=n$, to (1.31), when $\theta=p$; in this last case the $W^{1, p}$-regularity of the solution is missed just by a natural Marcinkiewicz-scale factor. A warning for the reader: in $\mathcal{M}^{q, \theta}$ the second exponent does not "tune" the first one; these are not like Lorentz spaces: indeed $\mathcal{M}^{q, 0} \equiv L^{\infty}$ and $\mathcal{M}^{q, n} \equiv \mathcal{M}^{q}$, for every $q \geq 1$. Finally, note that (1.29) does not require (1.4) since we are not dealing with higher derivatives of the gradient, and we do not need to "differentiate" equation (1.1) 1 , that obviously needs (1.4).

The second effect of condition (1.27) is an expansion of the range (1.15). The fractional derivatives are themselves in a Morrey space, see the definition in (2.16). This leads to the final and central stage of our regularity program:

Theorem 1.9 (Sobolev-Morrey regularity). Under the assumptions (1.2) and (1.4) with $p \leq n$, and (1.27) with $\theta \geq p$, let $u \in W_{0}^{1,1}(\Omega)$ be the solution found in Theorem 1.8. Then

$$
D u \in W_{\text {loc }}^{\frac{\sigma(q, \theta)-\varepsilon}{q}, q, \theta}\left(\Omega, \mathbb{R}^{n}\right),
$$

for every $\varepsilon \in(0, \sigma(q, \theta))$, where $m$ is in (1.28), and

$$
p-1 \leq q<\frac{\theta(p-1)}{\theta-1}=m, \quad \sigma(q, \theta):=\theta-\frac{q(\theta-1)}{p-1}=\theta(1-q / m) .
$$

In particular

$$
D u \in W_{\mathrm{loc}}^{\frac{1-\varepsilon}{p-1}, p-1, \theta}\left(\Omega, \mathbb{R}^{n}\right) \text {, and } D u \in W_{\mathrm{loc}}^{1-\varepsilon, 1, \theta}\left(\Omega, \mathbb{R}^{n}\right) \text { when } p=2 .
$$

Moreover, for any open subset $\Omega^{\prime} \subset \subset \Omega$ and $\sigma \in(0, \sigma(q, \theta))$, we have

$$
\|D u\|_{W^{\sigma / q, q, \theta}\left(\Omega^{\prime}\right)} \leq c M^{\frac{1}{p-1}}+c s|\Omega|^{\frac{1}{q}},
$$

where $c \equiv c\left(n, p, L / \nu, q, \sigma\right.$, $\left.\operatorname{dist}\left(\Omega^{\prime}, \partial \Omega\right), \Omega\right)$, and $M$ is in (1.27).

Originally introduced in $[15,16]$, Sobolev-Morrey spaces $W^{\alpha, q, \theta}$ appear in various forms in several pde issues as they provide the natural scaling properties of solutions $[47,58,71]$. Estimate (1.35) extends to the case of non-linear equations with measure data the classical Morrey space results for linear elliptic equations $[12,17,23,24,34,50]$; see the definition in (2.14) below. The standard result for the model case $\Delta v=f$ is that $D v \in W^{1, q, \theta}$ when $f \in L^{q, \theta}$ for $q>1$, that is

$$
\int_{B_{R}}\left|D^{2} v\right|^{q} d x \leq c R^{n-\theta} .
$$

Inclusion (1.34) sharply extends this to the case $q=1$, that is previous inequality is replaced by the following analogue:

$$
\int_{B_{R}} \int_{B_{R}} \frac{|D u(x)-D u(y)|}{|x-y|^{n+1-\varepsilon}} d x d y \leq c R^{n-\theta},
$$


which is valid for every $\varepsilon \in(0,1)$ and every ball $B_{R} \subset \subset \Omega$ of radius $R$, where $c$ depends on $\varepsilon$ and on the distance between $B_{R}$ and $\partial \Omega$. Finally, in light of (1.29) we can interpret (1.32) and therefore also (1.14) as a scale of regularity for $D u$ leading, as $q \nearrow m$, from the maximal differentiability (1.34) towards the maximal integrability (1.29).

\subsection{The capacitary case}

This is when $\theta<p$; this case is simpler and we will be shorter. Here $\mu \in W^{-1, p^{\prime}}$, that is, the dual space of $W^{1, p}$, and moreover $\mu$ cannot charge null $p$-capacity sets. When $p>n$ this follows from Sobolev's embedding theorem; one-point sets have positive $p$-capacity. When $\theta<p \leq n$ a basic theorem of $\mathrm{D}$. $\mathrm{R}$. Adams [1,3] still ensures that $\mu \in W^{-1, p^{\prime}}$; here note that (1.27) implies $|\mu|\left(B_{R}\right) \preceq R^{p-\theta} \operatorname{Cap}_{p}\left(B_{R}\right)$. At the end (1.1) can be solved by monotonicity methods [53], and the existence of a unique solution in the natural space $W_{0}^{1, p}(\Omega)$ follows.

Theorem 1.10 (Capacitary Calderón-Zygmund estimates). Assume (1.2), (1.4), and (1.27) with $\theta<p$. Then the unique solution $u \in W_{0}^{1, p}(\Omega)$ to the problem (1.1) satisfies

$$
D u \in W_{\mathrm{loc}}^{\frac{\sigma(p)-\varepsilon}{p}, p}\left(\Omega, \mathbb{R}^{n}\right), \quad \sigma(p):=\frac{p-\theta}{p-1},
$$

for every $\varepsilon \in(0, \sigma(p))$. Moreover, for any open subset $\Omega^{\prime} \subset \subset \Omega$ we have

$\int_{\Omega}|D u|^{p} d x+\int_{\Omega^{\prime}} \int_{\Omega^{\prime}} \frac{|D u(x)-D u(y)|^{p}}{|x-y|^{n+\sigma}} d x d y \leq c M^{\frac{1}{p-1}}|\mu|(\Omega)+c s^{p}|\Omega|$,

where $c \equiv c\left(n, p, L / \nu, \sigma, \theta, \operatorname{dist}\left(\Omega^{\prime}, \partial \Omega\right), \Omega\right)$, and $M$ appears in (1.27).

As a corollary of (1.36) and of the fractional Sobolev embedding Theorem 2.2, we also have the following higher integrability result:

$$
D u \in L_{\mathrm{loc}}^{t}\left(\Omega, \mathbb{R}^{n}\right) \quad \text { for every } t<\frac{n p}{n-\sigma(p)} .
$$

We point out the analogy between (1.36) and the results in [46] for the case $\theta<$ $p \leq n$, stating that solutions to (1.6) are $C^{0, \alpha}$-regular with $\alpha=\sigma(p)$; see also $[41,43,49]$. Theorem 1.10 extends to general elliptic systems, see Section 11.6.

\subsection{Additional results}

For the proof of the above theorems we shall need the following intermediate result, which may have its own interest; see also $[19,25]$ for a particular case. 
Theorem 1.11 (Morrey space regularity). Under the assumptions (1.2) with $p \leq$ $n$, and (1.27) with $\theta \geq p$, let $u \in W_{0}^{1,1}(\Omega)$ be the solution found in Theorem 1.8. Then Du belongs to the Morrey space $L_{\mathrm{loc}}^{q, \delta(q)}\left(\Omega, \mathbb{R}^{n}\right)$, for every $q$ and $\delta(q)$ such as

$$
1 \leq q<\frac{\theta(p-1)}{\theta-1}=m, \quad \delta(q):=\frac{q(\theta-1)}{p-1} .
$$

For every $\Omega^{\prime} \subset \subset \Omega$, there exists $c \equiv c\left(n, p, L / v, q\right.$, $\left.\operatorname{dist}\left(\Omega^{\prime}, \partial \Omega\right), \Omega\right)$ such that

$$
\|D u\|_{L^{q, \delta(q)}\left(\Omega^{\prime}\right)} \leq c M^{\frac{1}{p-1}}+c s|\Omega|^{\frac{1}{q}} .
$$

For the sake of completeness we also include a corollary that in different forms, but not in the following one, already appears in the literature $[27,31,73]$.

Theorem 1.12 (BMO/VMO regularity). Under the assumptions (1.2) with $p \leq$ $n$, and (1.27) with $\theta=p$, the solution $u \in W_{0}^{1,1}(\Omega)$ found in Theorem 1.8 belongs to $\mathrm{BMO}_{\text {loc }}(\Omega)$. Moreover, if

$$
\lim _{R \searrow 0} \frac{|\mu|\left(B_{R}\right)}{R^{n-p}}=0
$$

locally uniformly in $\Omega$, then $u \in \mathrm{VMO}_{\text {loc }}(\Omega)$. Finally, for every open subset $\Omega^{\prime} \subset \subset$ $\Omega$ there exists a constant $c \equiv c\left(n, p, L / \nu, \operatorname{dist}\left(\Omega^{\prime}, \partial \Omega\right), \Omega\right)$ such that

$$
[u]_{\mathrm{BMO}\left(\Omega^{\prime}\right)} \leq c M^{\frac{1}{p-1}}+c s|\Omega| .
$$

For the exact meaning of "locally uniformly" in (1.41) see Definition 2.6 below; see also Remark 2.7. Observe that also in this case the result complements the ones in the literature: as soon as $\theta<p$ solutions are Hölder continuous [41,49].

Remark 1.13. In Theorems 1.9-1.12 the constants $c$ depending on $q$, $\varepsilon$ blow-up as $q \nearrow m, \varepsilon \searrow 0, \sigma \nearrow \sigma(q, \theta) ; \sigma \nearrow \sigma(p)$ in case of Theorem 1.10.

Finally, a road-map to the paper. Some of the results presented are obtained via a delicate interaction between various types of regularity scales. For instance, as for Theorems 1.8 and Theorem 1.9, we have

$$
D u \in L_{\mathrm{loc}}^{q, \delta(q)}\left(\Omega, \mathbb{R}^{n}\right), q<b \Longrightarrow D u \in \mathcal{M}_{\mathrm{loc}}^{m, \theta} \Longrightarrow D u \in W_{\mathrm{loc}}^{\frac{\sigma(q, \theta)-\varepsilon}{q}, q, \theta} .
$$

In Section 2 we collect a miscellanea of preliminary material and notations. Section 3 includes some results for elliptic problems, that in the form presented are not explicitly contained in the literature. In Section 4 we collect a few preparatory lemmas of comparison type, while in Section 5 we fix the basic approximation procedure. Section 6 contains the proofs of Theorems 1.2-1.4 and Corollary 1.5, while Section 7 contains the one of Theorem 1.10. Section 8 contains the proof 
of Theorems 1.11 and 1.12. Section 9 contains the proof of Theorem 1.8, while Section 10 contains the one of Theorem 1.9. Finally, in Section 11 we discuss the sharpness of some of the results obtained.

Part of the results obtained in this paper have been announced in the preliminary Comptes Rendus note [60].

Acknowledgements. Part of this paper was carried out while the author was visiting FIM at ETH Zürich in January 2006, and the Institut für Mathematik at Humboldt University Berlin in April 2006, institutions that provided a nice and stimulating environment; thanks go to Tristan Rivière and Barbara Niethammer, respectively, for their invitations. Thanks also go to Nicola Fusco, Jan Kristensen, Francesco Leonetti, Juan J. Manfredi for stimulating conversations on problems with measure data; special thanks finally go to Anna Föglein and Tero Kilpeläinen for a careful reading of an earlier version of the paper.

\section{Preliminaries, function spaces}

\subsection{General notation}

In this paper we shall adopt the usual, but somehow arguable convention to denote by $c$ a general constant, that may vary from line to line; peculiar dependence on parameters will be properly emphasized in parentheses when needed, while special occurences will be denoted by $c_{*}, c_{1}, c_{2}$ or the like. With $x_{0} \equiv\left(x_{0,1}, \ldots, x_{0, n}\right) \in$ $\mathbb{R}^{n}$, we denote

$$
B_{R}\left(x_{0}\right) \equiv B\left(x_{0}, R\right):=\left\{x \in \mathbb{R}^{n}:\left|x-x_{0}\right|<R\right\}
$$

and

$$
Q_{R}\left(x_{0}\right) \equiv Q\left(x_{0}, R\right):=\left\{x \in \mathbb{R}^{n}: \sup \left|x_{i}-x_{0, i}\right|<R, 1 \leq i \leq n\right\},
$$

the open ball and cube, respectively, with center $x_{0}$ and "radius" $R$. We shall often use the short hand notation $B_{R} \equiv B\left(x_{0}, R\right)$ and $Q_{R} \equiv Q\left(x_{0}, R\right)$, when no ambiguity will arise. Moreover, with $B, Q$ being balls and cubes, respectively, by $\gamma B, \gamma Q$ we shall denote the concentric balls and cubes, with radius magnified by a factor $\gamma$. If $g: A \rightarrow \mathbb{R}^{k}$ is an integrable map with respect to the Borel measure $\mu$, and $0<\mu(A)<\infty$, we write

$$
(g)_{\mu, A}:=f_{A} g(x) d \mu:=\frac{1}{\mu(A)} \int_{A} g(x) d \mu .
$$

When $\mu$ is the Lebesgue measure and $A \equiv B\left(x_{0}, R\right)$, we may also use the short hand notation $(g)_{\mu, A} \equiv(g)_{A} \equiv(g)_{B_{R}} \equiv(g)_{B}$. 
Permanent conventions. In the estimates the constants will in general depend on the parameters $n, p, v, L$. The dependence on $v, L$ is actually via the ellipticity ratio $L / v$, and will be given directly in this way. This can be seen by passing to rescaled vector fields $a / \nu$. When considering a function space $X\left(\Omega, \mathbb{R}^{k}\right)$ of possibly vector valued measurable maps defined on an open set $\Omega \subset \mathbb{R}^{n}$, with $k \in \mathbb{N}$, e.g.: $L^{p}\left(\Omega, \mathbb{R}^{k}\right), W^{\alpha, p}\left(\Omega, \mathbb{R}^{k}\right)$, we shall define in a canonic way the local variant $X_{\text {loc }}\left(\Omega, \mathbb{R}^{k}\right)$ as that space of maps $f: \Omega \rightarrow \mathbb{R}^{k}$ such that $f \in X\left(\Omega^{\prime}, \mathbb{R}^{k}\right)$, for every $\Omega^{\prime} \subset \subset \Omega$. Moreover, also in the case $f$ is vector valued, that is $k>1$, we shall also use the short hand notation $X\left(\Omega, \mathbb{R}^{k}\right) \equiv X(\Omega)$, or even $X\left(\Omega, \mathbb{R}^{k}\right) \equiv X$.

\subsection{The map $V(z)$, and the monotonicity of $a(x, z)$}

With $s \geq 0$, we define

$$
V(z)=V_{s}(z):=\left(s^{2}+|z|^{2}\right)^{\frac{p-2}{4}} z, \quad z \in \mathbb{R}^{n},
$$

which is easily seen to be a locally bi-Lipschitz bijection of $\mathbb{R}^{n}$. A basic property of $V$, whose proof can be found in [36], Lemma 2.1, is the following: For any $z_{1}, z_{2} \in \mathbb{R}^{n}$, and any $s \geq 0$, it holds

$$
c^{-1}\left(s^{2}+\left|z_{1}\right|^{2}+\left|z_{2}\right|^{2}\right)^{\frac{p-2}{2}} \leq \frac{\left|V\left(z_{2}\right)-V\left(z_{1}\right)\right|^{2}}{\left|z_{2}-z_{1}\right|^{2}} \leq c\left(s^{2}+\left|z_{1}\right|^{2}+\left|z_{2}\right|^{2}\right)^{\frac{p-2}{2}},
$$

where $c \equiv c(n, p)$, is independent of $s$. We also notice that

$$
|z|^{p} \leq|V(z)|^{2} \leq 2\left(s^{p}+|z|^{p}\right) .
$$

Indeed when $p=2$ this is trivial, otherwise when $p>2$ we just use Young's inequality with conjugate exponents $(p, p /(p-2))$; in what follows we shall also need another elementary property of $V$ :

$$
V_{s / A}(z / A)=A^{-p / 2} V_{s}(z), \quad \text { for every } s \geq 0, \text { and } A>0 .
$$

The strict monotonicity properties of the vector field $a$ implied by the left-hand side in (1.2) $)_{1}$ can be recast using the map $V$. Indeed combining (1.2) $)_{1}$ and (2.2) yields, for $c \equiv c(n, p, v)>0$, and whenever $z_{1}, z_{2} \in \mathbb{R}^{n}$

$$
c^{-1}\left|V\left(z_{2}\right)-V\left(z_{1}\right)\right|^{2} \leq\left\langle a\left(x, z_{2}\right)-a\left(x, z_{1}\right), z_{2}-z_{1}\right\rangle .
$$

Moreover, since $p \geq 2$, assumption (1.2) 1 also implies

$$
c^{-1}\left|z_{2}-z_{1}\right|^{p} \leq\left\langle a\left(x, z_{2}\right)-a\left(x, z_{1}\right), z_{2}-z_{1}\right\rangle .
$$

Finally, inequality $(1.2)_{1}$, together with (1.2) $)_{3}$ and a standard use of Young's inequality, yield for every $z \in \mathbb{R}^{n}$

$$
c^{-1}\left(s^{2}+|z|^{2}\right)^{\frac{p-2}{2}}|z|^{2}-c s^{p} \leq\langle a(x, z), z\rangle, \quad c \equiv c(n, p, L / v),
$$


while (1.2) 2 and again (1.2) 3 give, again via Young's inequality

$$
|a(x, z)| \leq c\left(s^{2}+|z|^{2}\right)^{\frac{p-1}{2}} .
$$

In the following and for the rest of the paper, unless otherwise stated, we shall denote $V \equiv V_{s}$ with $s$ fixed at the beginning, in (1.3).

\subsection{Fractional Sobolev/Nikolski spaces, and difference operators}

We recall some basic facts about fractional order Sobolev spaces, using the standard notation from [4], adapted to the situations we are going to deal with. For a bounded open set $A \subset \mathbb{R}^{n}$ and $k \in \mathbb{N}$, parameters $\alpha \in(0,1)$ and $q \in[1, \infty)$, we write $w \in W^{\alpha, q}\left(A, \mathbb{R}^{k}\right)$ provided the following Gagliardo-type norm is finite:

$$
\begin{aligned}
\|w\|_{W^{\alpha, q}(A)} & :=\left(\int_{A}|w(x)|^{q} d x\right)^{\frac{1}{q}}+\left(\int_{A} \int_{A} \frac{|w(x)-w(y)|^{q}}{|x-y|^{n+\alpha q}} d x d y\right)^{\frac{1}{q}} \\
& =:\|w\|_{L^{q}(A)}+[w]_{\alpha, q ; A}<\infty .
\end{aligned}
$$

For a possibly vector valued function $w: \Omega \rightarrow \mathbb{R}^{k}$, and a real number $h \in \mathbb{R}$, we define the finite difference operator $\tau_{i, h}$ for $i \in\{1, \ldots, n\}$ as

$$
\tau_{i, h} w(x) \equiv \tau_{i, h}(w)(x):=w\left(x+h e_{i}\right)-w(x) .
$$

where $\left\{e_{i}\right\}_{1 \leq i \leq n}$ denotes the standard basis of $\mathbb{R}^{n}$. This makes sense whenever $x, x+h e_{i} \in A$, an assumption that will be satisfied whenever we use $\tau_{i, h}$ in the following. In particular, we shall very often take $x \in A$ where $A \subset \subset \Omega$ is an open subset of $\Omega$, and where $|h| \leq \operatorname{dist}(A, \partial \Omega)$. Accordingly, the Nikolski space $\mathcal{N}^{\alpha, q}(A)$, with $A \subset \subset \Omega$ is hereby defined by saying that $u \in \mathcal{N}^{\alpha, q}(A)$ if and only if

$$
\|w\|_{\mathcal{N}^{\alpha, q}(A)}:=\|w\|_{L^{q}(A)}+\sum_{i=1}^{n} \sup _{h}|h|^{-\alpha}\left\|\tau_{i, h} w\right\|_{L^{q}(A)},
$$

is finite, where $0<|h| \leq \operatorname{dist}(A, \partial \Omega)$. In the following we shall also let $W^{0, q} \equiv$ $\mathcal{N}^{0, q} \equiv L^{q}$. The strict inclusions

$$
W^{\alpha, q}(A) \subset \mathcal{N}^{\alpha, q}(A) \subset W^{\alpha-\varepsilon, q}(A), \quad \forall \varepsilon \in(0, \alpha),
$$

are well known, and the next lemma somehow quantifies the last one.

Lemma 2.1. Let $w \in L^{q}\left(\Omega, \mathbb{R}^{k}\right), q>1$, and assume that for $\bar{\alpha} \in(0,1], S \geq 0$ and an open set $\tilde{\Omega} \subset \subset \Omega$ we have

$$
\left\|\tau_{i, h} w\right\|_{L^{q}(\tilde{\Omega})} \leq S|h|^{\bar{\alpha}},
$$

for every $1 \leq i \leq n$ and every $h \in \mathbb{R}$ satisfying $0<|h| \leq d$, where $0<d \leq$ $\min \{1, \operatorname{dist}(\tilde{\Omega}, \partial \Omega)\}$. Then $w \in W_{\text {loc }}^{\alpha, q}\left(\tilde{\Omega}, \mathbb{R}^{k}\right)$ for every $\alpha \in(0, \bar{\alpha})$, and for each 
open set $A \subset \subset \tilde{\Omega}$ there exists a constant $c \equiv c(d, \bar{\alpha}-\alpha, \operatorname{dist}(A, \partial \tilde{\Omega}))$, independent of $S$ and $w$, such that

$$
\|w\|_{W^{\alpha, q}\left(A, \mathbb{R}^{k}\right)} \leq c\left(S+\|w\|_{L^{q}\left(A, \mathbb{R}^{k}\right)}\right) .
$$

Basic references for the last result are [4, Chapter 7], or [48]; see also [30, Lemma 3 ], from which the previous lemma follows via a covering argument. The following result is nothing but Sobolev's embedding theorem in the case of fractional spaces; see again [4] and also [30, Lemma 3].

Theorem 2.2 (Fractional Sobolev embedding). Let $w \in W^{\alpha, q}\left(A, \mathbb{R}^{k}\right)$, with $q \geq$ 1 and $\alpha \in(0,1]$, such that $\alpha q<n$, and let $A \subset \mathbb{R}^{n}$ be a Lipschitz domain. Then $w \in L^{n q /(n-\alpha q)}\left(A, \mathbb{R}^{k}\right)$, and there exists a constant $c \equiv c\left(n, \alpha, q,[\partial A]_{0,1}\right)$ such that

$$
\|w\|_{L^{\frac{n q}{n-\alpha q}}(A)} \leq c\|w\|_{W^{\alpha, q}(A)} .
$$

For the following fact see for instance [59] and related references.

Proposition 2.3 (Fractional Poincaré inequality). If $B \equiv B\left(x_{0}, R\right) \subset \mathbb{R}^{n}$ is $a$ ball and $w \in W^{\alpha, q}\left(B, \mathbb{R}^{k}\right)$, then

$$
\int_{B}\left|w-w_{B}\right|^{q} d x \leq c(n) R^{\alpha q} \int_{B} \int_{B} \frac{|w(x)-w(y)|^{q}}{|x-y|^{n+\alpha q}} d x d y=c(n) R^{\alpha q}[w]_{\alpha, q ; B}^{q} .
$$

The following result is classical in potential theory [3]; see again [59] for an elementary proof that avoids potential theory.

Proposition 2.4. Let $w \in W_{\mathrm{loc}}^{\alpha, q}\left(\Omega, \mathbb{R}^{k}\right)$, where $0<\alpha<1, q \geq 1$ are such that $\alpha q<n$. Let $\Sigma_{w}$ denote the set of non-Lebesgue points of $w$ in the sense of

$$
\Sigma_{w}:=\left\{x \in \Omega: \liminf _{\rho \searrow 0} f_{B(x, \rho)}\left|w(y)-(w)_{x, \rho}\right|^{q} d y>0 \text { or } \limsup _{\rho \searrow 0}\left|(w)_{x, \rho}\right|=\infty\right\} .
$$

Then its Hausdorff dimension $\operatorname{dim}\left(\Sigma_{w}\right)$ satisfies $\operatorname{dim}\left(\Sigma_{w}\right) \leq n-\alpha q$.

\subsection{Morrey spaces, BMO, VMO}

We shall adopt a slightly modified definition of Morrey spaces, or more precisely: there are several possible, essentially equivalent definitions in the literature; we choose one. With $A \subset \mathbb{R}^{n}$ being an open subset, we define the Morrey space $L^{q, \theta}(A)$, with $q \geq 1$ and $\theta \in[0, n]$ as that of those measurable maps $w \in L^{q}(A)$ such that the following quantity is finite:

$$
\|w\|_{L^{q, \theta}(A)}^{q}:=\sup _{B_{R} \subset A, R \leq 1} R^{\theta-n} \int_{B_{R}}|w|^{q} d x .
$$


In the following, when considering the space $\mathcal{M}(A)$ of Borel measures with finite mass on $A \subset \mathbb{R}^{n}$, we shall automatically consider them extended on the whole $\mathbb{R}^{n}$ in the trivial way: $|\mu|\left(\mathbb{R}^{n} \backslash A\right):=0$. When considering $L^{1, \theta}(A)$, as in [2], we include measures $\mu \in \mathcal{M}(A)$ defining in this case

$$
\|\mu\|_{L^{1, \theta}(A)}:=\sup _{B_{R} \subset A, R \leq 1} R^{\theta-n}|\mu|\left(B_{R}\right)<\infty,
$$

and actually $L^{1, \theta}(A)$ will be considered as a subspace of $\mathcal{M}(A)$. Trivially, if $\mu$ satisfies (1.27) then $\mu \in L^{1, \theta}(A)$ for every open subset $A \subset \mathbb{R}^{n}$ and $\|\mu\|_{L^{1, \theta}(A)} \leq$ $M$. Information on Morrey spaces are in $[1,34]$. Our definition differs from the usual one in that we consider only balls contained in $A$ when stating (2.14), and with radius not larger than one, because we shall treat interior regularity, and information near the boundary $\partial \Omega$ will play no role. Such a modification is truly inessential, and will simplify the already heavy technical treatment in the following pages; observe that our definition is anyway consistent with the one in [69], Definition 1.1.

The following lemma is elementary and can be obtained via a standard scaling argument; the simple proof is left to the reader.

Lemma 2.5. Let $g \in L^{q, \theta}\left(B\left(x_{0}, r\right)\right)$ and define $\tilde{g}(y):=g\left(x_{0}+r y\right)$ for $y \in$ $B(0,1) \equiv B_{1}$. Then $\tilde{g} \in L^{q, \theta}\left(B_{1}\right)$ and $\|\tilde{g}\|_{L^{q, \theta}\left(B_{1}\right)}=r^{-\theta / q}\|g\|_{L^{q, \theta}\left(B\left(x_{0}, r\right)\right)}$.

We now pass to recall the definition of BMO and VMO spaces, introduced in $[40,66]$ respectively. As already in the case of Morrey spaces, we shall also modify a bit the definition in order to adapt it to the local statement we are giving in the following. The space $\mathrm{BMO}(\mathrm{A})$ is that of those measurable maps $w: A \rightarrow \mathbb{R}^{n}$ such that the semi-norm

$$
[w]_{\mathrm{BMO}(\mathrm{A})}:=\sup _{B_{R} \subset A} f_{B_{R}}\left|w-(w)_{B_{R}}\right| d x
$$

is finite. Further information can be found for instance in [34], and its references. Finally the space $\operatorname{VMO}_{\text {loc }}(\Omega)$. Let $\Omega^{\prime} \subset \subset \Omega$ be an open subset, and define

$$
\omega_{w}\left(R, \Omega^{\prime}\right):=\sup _{B_{r}, r \leq R} f_{B_{r}}\left|w-(w)_{B_{r}}\right| d x
$$

where $B_{r} \subset \subset \Omega$ is centered in $\Omega^{\prime}$. Then a map $w: \Omega \rightarrow \mathbb{R}^{n}$ belongs to $\operatorname{VMO}_{\text {loc }}(\Omega)$ if and only if $\lim _{R \searrow 0} \omega_{w}\left(R, \Omega^{\prime}\right)=0$ for every open subset $\Omega^{\prime} \subset \subset \Omega$. In connection to VMO spaces we shall need the following:

Definition 2.6. A Borel measure $\mu \in \mathcal{M}(\Omega)$ is said to satisfy the condition in (1.41) locally uniformly in $\Omega$ iff for every $\Omega^{\prime} \subset \subset \Omega$ and every $\varepsilon>0$ there exists $\bar{R}>0$, depending on $\varepsilon$ and $\operatorname{dist}\left(\Omega^{\prime}, \partial \Omega\right)$, such that $|\mu|\left(B_{R}\right) \leq \varepsilon R^{n-p}$, whenever $B_{R} \subset \Omega^{\prime}$ and $R \leq \bar{R}$.

Remark 2.7. When $p=n$ it is a simple exercise in basic measure theory to check that the measure $\mu$ fulfills Definition 2.6 iff $\mu$ has no atoms, i.e.: $\mu\left(\left\{x_{0}\right\}\right)=0$ for every $x_{0} \in \Omega$. This allows to view the local VMO regularity results of [31] as a particular case of Theorem 1.12. 


\subsection{Sobolev-Morrey spaces}

Beside that of Morrey spaces, we recall the definition of Sobolev-Morrey spaces of fractional order; also in this case we propose an inessential modification of the usual definition to simplify the treatment in the following. Let $A \subset \mathbb{R}^{n}$ be an open subset; we say that a map $w \in W^{\alpha, q}\left(A, \mathbb{R}^{k}\right)$, belongs to $W^{\alpha, q, \theta}\left(A, \mathbb{R}^{k}\right)$, with $\alpha \in(0,1]$, $q \in[1, \infty), \theta \in[0, n]$, iff $w \in W^{\alpha, q}\left(A, \mathbb{R}^{k}\right)$, and moreover

$$
[w]_{\alpha, q, \theta ; A}^{q}:=\left\{\begin{array}{cl}
\sup _{B_{R} \subset A, R \leq 1} R^{\theta-n}[w]_{\alpha, q ; B_{R}}^{q}<\infty & \text { if } \alpha<1 \\
\|D w\|_{L^{q, \theta}(A)}^{q} & \text { if } \alpha=1
\end{array}<\infty .\right.
$$

In any case we let

$$
\|w\|_{W^{\alpha, q, \theta}(A)}:=\|w\|_{W^{\alpha, q}(A)}+[w]_{\alpha, q, \theta ; A} .
$$

For such spaces and generalizations, see the original papers $[15,16]$ and $[58,71]$.

\subsection{Marcinkiewicz spaces}

Finally, Marcinkiewicz spaces $\mathcal{M}^{t}\left(A, \mathbb{R}^{k}\right), t \geq 1$, also called Lorentz-Marcinkiewicz spaces and denoted by $L^{t, \infty}(A)$, or by $L_{w}^{t}(A)$, when they are called "weak- $L^{t}$ " spaces. A measurable map $w: A \rightarrow \mathbb{R}^{k}$ belongs to $\mathcal{M}^{t}\left(A, \mathbb{R}^{k}\right)$ iff

$$
\sup _{\lambda \geq 0} \lambda^{t}|\{x \in A:|w|>\lambda\}|=:\|w\|_{\mathcal{M}^{t}(A)}^{t}<\infty .
$$

Yet, we recall the definition of Marcinkiewicz-Morrey spaces [2]. A map $w \in$ $\mathcal{M}^{t}\left(A, \mathbb{R}^{k}\right)$ belongs to the space $\mathcal{M}^{t, \theta}\left(A, \mathbb{R}^{k}\right)$ with $\theta \in[0, n]$ iff

$$
\sup _{B_{R} \subset A, R \leq 1} R^{\theta-n}\|w\|_{\mathcal{M}^{t}\left(B_{R}\right)}^{t}<\infty .
$$

Accordingly, we let

$$
\|w\|_{\mathcal{M}^{t, \theta}(A)}:=\|w\|_{\mathcal{M}^{t}(A)}+\left[\sup _{B_{R} \subset A, R \leq 1} R^{\theta-n}\|w\|_{\mathcal{M}^{t}\left(B_{R}\right)}^{t}\right]^{1 / t} .
$$

Obviously

$$
\|w\|_{\mathcal{M}^{t, n}(A)} \approx\|w\|_{\mathcal{M}^{t}(A)}, \quad \mathcal{M}^{t, n}(A) \equiv \mathcal{M}^{t}(A),
$$

and, according to the definition in (2.14)

$$
\mathcal{M}^{t, \theta_{1}}(A) \subset \mathcal{M}^{t, \theta_{2}}(A) \Longleftrightarrow \theta_{1}<\theta_{2}, \quad \text { with } \quad\|w\|_{\mathcal{M}^{t, \theta_{2}(A)}} \leq\|w\|_{\mathcal{M}^{t, \theta_{1}}(A)} .
$$


Lemma 2.8 (Hölder's inequality in $\mathcal{M}^{t}$ ). Let $f \in \mathcal{M}^{t}(A)$ with $t>1$. Then $f \in$ $L^{q}(A)$ with $1 \leq q<t$ and it holds

$$
\|f\|_{L^{q}(A)} \leq\left(\frac{t}{t-q}\right)^{\frac{1}{q}}|A|^{\frac{1}{q}-\frac{1}{t}}\|f\|_{\mathcal{M}^{t}(A)} .
$$

The proof of the latter result is standard [27,73]. Next, a so called "trace type inequality" $[1,55]$.

Theorem 2.9. Let $\lambda$ be a non-negative Radon measure on $\mathbb{R}^{n}$ such that $\lambda\left(B_{R}\right) \leq$ $M R^{n-\theta}$, for every ball $B_{R} \subset \mathbb{R}^{n}$, where $0 \leq \theta<p \leq n$ and $M>0$. Then when $p<n$ for every $w \in W_{0}^{1, p}\left(B_{R}\right)$ we have

$$
\int_{B_{R}}|w|^{p} d \lambda \leq c M R^{p-\theta} \int_{B_{R}}|D w|^{p} d x
$$

where $c \equiv c(n, p, \theta)$. In the limit case $p=n$ inequality (2.21) holds replacing $c M R^{n-\theta}$ by $c M R^{\sigma}$, for any $\sigma<n-\theta$, where $c \equiv c(n, \theta, \sigma)$.

Proof. We did not find any direct reference for this result, therefore we sketch the proof for the reader's convenience, based on the results in [1]. Firstly the case $p<n$. Letting

$$
p_{\theta}:=\frac{(n-\theta) p}{n-p}, \quad \text { and } \quad \tilde{\lambda}:=\lambda / M
$$

we obviously have $\tilde{\lambda}\left(B_{R}\right) \leq R^{n-\theta}$, and then it holds

$$
\left(\int_{B_{R}}|w|^{p_{\theta}} d \tilde{\lambda}\right)^{\frac{1}{p_{\theta}}} \leq c(n, p)\left(\int_{B_{R}}|D w|^{p} d x\right)^{\frac{1}{p}}
$$

This is Adams' inequality, see [55, Corollary 1.93]; see also [3, comments at Chapter 7] to see the earlier contributions of Mazy'a, and the original paper of Adams [1]. Using Hölder's inequality, as $p_{\theta} \geq p$, we have

$$
\left(f_{B_{R}}|w|^{p} d \tilde{\lambda}\right)^{\frac{1}{p}} \leq\left(f_{B_{R}}|w|^{p_{\theta}} d \tilde{\lambda}\right)^{\frac{1}{p_{\theta}}} \leq c \tilde{\lambda}\left(B_{R}\right)^{-\frac{1}{p_{\theta}}}\left(\int_{B_{R}}|D w|^{p} d x\right)^{\frac{1}{p}}
$$

therefore, using again that $\tilde{\lambda}\left(B_{R}\right) \leq R^{n-\theta}$ and (2.22) we have

$$
\int_{B_{R}}|w|^{p} d \tilde{\lambda} \leq c \tilde{\lambda}\left(B_{R}\right)^{\frac{p-\theta}{n-\theta}} \int_{B_{R}}|D w|^{p} d x \leq c R^{p-\theta} \int_{B_{R}}|D w|^{p} d x,
$$

and (2.21) follows scaling back to $\lambda$. Now we treat the case $p=n$. In this case observe that $\tilde{\lambda}\left(B_{R}\right) \leq c \log ^{q(1-n) / n}\left(R^{-1}\right)$ when $R \leq 1 / 2$, and $\tilde{\lambda}\left(B_{R}\right) \leq 2^{\sigma-q} R^{q}$ 
when $R>1 / 2$, where $q>n$, and $c \equiv(n, p, \theta, q)$ is a suitable constant. Therefore we may apply Theorem 1.94 from [55], see also [3], Theorem 7.2.2, to have

$$
\left(\int_{B_{R}}|w|^{q} d \lambda\right)^{\frac{1}{q}} \leq c(n, p, \theta, q)\left(\int_{B_{R}}|D w|^{n} d x\right)^{\frac{1}{n}} .
$$

Applying Hölder's inequality and $\tilde{\lambda}\left(B_{R}\right) \leq R^{n-\theta}$ yields, with $c \equiv c(n, p, \theta, q)$

$$
\int_{B_{R}}|w|^{n} d \lambda \leq c(n, q, \sigma) \tilde{\lambda}\left(B_{R}\right)^{\frac{q-n}{q}} \int_{B_{R}}|D w|^{n} d x \leq c R^{(n-\theta)\left(1-\frac{n}{q}\right)} \int_{B_{R}}|D w|^{n} d x .
$$

The assertion follows taking $q \equiv q(\sigma)$ large enough, and scaling back to $\lambda$.

Remark 2.10. In a similar way, if $w \in W_{0}^{1, p}(\Omega)$ with $c \equiv c(n, p, \theta, \Omega)$ we have

$$
\int_{\Omega}|w|^{p} d \lambda \leq c M \int_{\Omega}|D w|^{p} d x
$$

\subsection{Technical lemmata}

The following is a simple variant of a well known iteration result. See for instance [34, Chapter 7], or [73, last section].

Lemma 2.11. Let $\phi:[0, \bar{R}] \rightarrow[0, \infty)$ be a non-decreasing function such that

$$
\phi(\varrho) \leq c_{0}\left(\frac{\varrho}{R}\right)^{\delta_{0}} \varphi(R)+\mathcal{B} R^{\gamma}, \quad \text { for every } \varrho<R \leq \bar{R}, \quad \mathcal{B} \geq 0,
$$

where $\gamma \in\left(0, \delta_{0}\right)$. Then if $\delta_{1} \in\left[\gamma, \delta_{0}\right)$, there exists $c_{1} \equiv c_{1}\left(c_{0}, \delta_{1}, \gamma\right)$ such that

$$
\phi(\varrho) \leq c_{1}\left(\frac{\varrho}{R}\right)^{\delta_{1}} \varphi(R)+c_{1} \mathcal{B} \varrho^{\gamma}, \quad \text { for every } \varrho \leq R \leq \bar{R} .
$$

Then, Giaquinta \& Giusti's “simple but fundamental lemma”, [34, Chapter 6].

Lemma 2.12. Let $\varphi:\left[R_{0}, 2 R_{0}\right] \rightarrow[0, \infty)$ be a function such that

$$
\varphi(t) \leq(1 / 2) \varphi(\varrho)+\mathcal{B}(\varrho-t)^{-\beta}+K, \quad \text { for every } R_{0}<t<\varrho<2 R_{0},
$$

where $\mathcal{B}, K \geq 0$ and $\beta>0$. Then $\varphi\left(R_{0}\right) \leq c(\beta) \mathcal{B} R_{0}^{-\beta}+c K$.

Finally, a standard fact.

Lemma 2.13. Let $\Omega^{\prime} \subset \subset \Omega \subset \mathbb{R}^{n}$ be bounded domains. There exists another open subset $\Omega^{\prime \prime}$ such that $\Omega^{\prime} \subset \subset \Omega^{\prime \prime} \subset \subset \Omega$ and

$$
\operatorname{dist}\left(\Omega^{\prime}, \partial \Omega^{\prime \prime}\right)=\operatorname{dist}\left(\Omega^{\prime \prime}, \partial \Omega\right)=\operatorname{dist}\left(\Omega^{\prime}, \partial \Omega\right) / 2 .
$$

Proof. Just let $\Omega^{\prime \prime}:=\left\{x \in \Omega: \operatorname{dist}\left(x, \overline{\Omega^{\prime}}\right)<1 / 2\right\}$. 


\section{Regularity for homogeneous problems}

In this section we recall some results on the regularity of solutions to homogeneous elliptic systems and equations with $p$-growth; some of them are well-known; some others, much less if not at all, especially in the explicit form needed in this paper. In such cases we shall give - sometimes sketchy - proofs; anyway a good general reference for the whole section is [34, Chapters 6 and 7].

Let us start with a simple but rather rarely used lemma on reverse Hölder inequalities. For the proof it suffices to follow in [34, Remark 6.12, page 205]; see also [11] for this kind of result.

Lemma 3.1. Let $g: A \rightarrow \mathbb{R}^{k}$ be a measurable map, and $\chi_{0}>1, c, s \geq 0$, such that

$$
\left(f_{B_{R}}|g|^{\chi_{0}} d x\right)^{\frac{1}{\chi_{0}}} \leq c f_{B_{2 R}}(|g|+s) d x,
$$

whenever $B_{2 R} \subset \subset A$, where $A \subset \mathbb{R}^{n}$ is an open subset. Then, for every $t \in(0,1]$ there exists a constant $c_{0} \equiv c_{0}(n, c, t)$ such that, for every $B_{2 R} \subseteq A$

$$
\left(f_{B_{R}}|g|^{\chi_{0}} d x\right)^{\frac{1}{\chi_{0}}} \leq c_{0}\left(f_{B_{2 R}}\left(|g|^{t}+s^{t}\right) d x\right)^{\frac{1}{t}} .
$$

The next two lemmata will be of fundamental importance in the following in that they provide estimates below the natural growth exponent $p$. For reasons that will become clear in Section 11 the first one is stated directly for systems, that is when $u$ is a vector valued map and therefore $N \geq 1$.

Lemma 3.2. Let $v_{0} \in W^{1, p}\left(A, \mathbb{R}^{N}\right)$ be a weak solution to the system

$$
\operatorname{div} a_{0}\left(D v_{0}\right)=0 \quad \text { in } A \text {. }
$$

Here $a_{0}: \mathbb{R}^{N \times n} \rightarrow \mathbb{R}^{N \times n}$ satisfies the assumptions (1.2) obviously recast to fit the vectorial case with no $x$-dependence, and $A \subset \mathbb{R}^{n}$ is an open subset. Then $V\left(D v_{0}\right) \in W_{\mathrm{loc}}^{1,2}\left(A, \mathbb{R}^{N \times n}\right)$, and there exists $c \equiv c(n, N, p, L / v)$ such that for every $z_{0} \in \mathbb{R}^{N \times n}$ and every ball $B_{R} \subseteq A$, we have

$$
\int_{B_{R / 2}}\left|D\left(V\left(D v_{0}\right)\right)\right|^{2} d x \leq \frac{c}{R^{2}} \int_{B_{R}}\left|V\left(D v_{0}\right)-V\left(z_{0}\right)\right|^{2} d x .
$$

Moreover, for every $t \in(0,1]$ there exists $c \equiv c(n, N, p, L / v, t)$ such that

$$
\left(f_{B_{R / 2}}\left|V\left(D v_{0}\right)-V\left(z_{0}\right)\right|^{2} d x\right)^{\frac{1}{2}} \leq c\left(f_{B_{R}}\left|V\left(D v_{0}\right)-V\left(z_{0}\right)\right|^{2 t} d x\right)^{\frac{1}{2 t}} .
$$

All the constants named $c$ involved in (3.1)-(3.2) are independent of the choice of $z_{0} \in \mathbb{R}^{N \times n}$. 


\section{Proof.}

Step 1: Regularization. We first regularize the problem following a few smoothing arguments similar to those in [29]. We consider a standard, symmetric and nonnegative mollifier $\phi: \mathbb{R}^{N \times n} \rightarrow \mathbb{R}$, such that $\phi \in C_{0}^{\infty}\left(B_{1}\right)$, and $\|\phi\|_{L^{1}\left(\mathbb{R}^{N \times n}\right)}=1$. Moreover, in order to apply the technique of [29] we also need a technical assumption, namely we need to take $\phi$ such that

$$
\int_{B_{1} \backslash B_{1 / 2}} \phi(z) d z \geq 1 / 1000
$$

For every $k \in \mathbb{N}$ set $\phi_{k}(z):=k^{N \times n} \phi(k z)$, and then define the smooth vector field $a_{k}(z)$ via convolution as follows:

$$
a_{k}(z):=\left(a_{0} * \phi_{k}\right)(z):=\int_{B(0,1)} a_{0}\left(z+k^{-1} y\right) \phi(y) d y
$$

Assumptions (1.2) and a few convolution estimates also using (3.3), similar to those of [29], Lemma 3.1, imply that each $a_{k}$ satisfies

$$
\left\{\begin{array}{l}
\left|a_{k}(z)\right|+\left|\partial_{z} a_{k}(z)\right|\left(s_{k}^{2}+|z|^{2}\right)^{\frac{1}{2}} \leq c\left(s_{k}^{2}+|z|^{2}\right)^{\frac{p-1}{2}} \\
c^{-1}\left(s_{k}^{2}+|z|^{2}\right)^{\frac{p-2}{2}}|\lambda|^{2} \leq\left\langle\partial_{z} a_{k}(z) \lambda, \lambda\right\rangle \\
\left|a_{0}(z)-a_{k}(z)\right| \leq c k^{-1}\left(s_{k}^{2}+|z|^{2}\right)^{\frac{p-2}{2}}
\end{array}\right.
$$

whenever $z, \lambda \in \mathbb{R}^{N \times n}$, where $s_{k}:=s+k^{-1}, k \in \mathbb{N}$, and $c \equiv c(n, N, p, L / \nu)$. Note that the new ellipticity/growth constant $c$ is actually independent of $k \in \mathbb{N}$. Moreover each $a_{k}$ satisfies the assumptions (1.2) with $s$ replaced by $s_{k}$, for different constants $v, L$, but still depending on the original ones. This fact and standard monotonicity methods [53] allow to define, with $B_{R} \subset \subset \Omega$ as in the statement, $v_{k} \in v_{0}+W_{0}^{1, p}\left(B_{R}\right)$ as the unique solution to

$$
\begin{cases}-\operatorname{div} a_{k}\left(D v_{k}\right)=0 & \text { in } B_{R} \\ v_{k}=v_{0} & \text { on } \partial B_{R}\end{cases}
$$

Step 2: Estimates. Under assumptions (3.4) 1,2 the proof of Caccioppoli's type inequality (3.1) with $c \equiv c(n, N, p, L / v), V\left(D v_{0}\right) \equiv V_{s_{k}}\left(D v_{k}\right)$, and any ball $B_{r} \subseteq B_{R}$, can be inferred from [18], Theorem 1.1, with minor variants, see also $[29,36,48]$. As for the proof of (3.2), set

$$
\chi_{0}:=\left\{\begin{array}{cl}
\frac{n}{n-2} & \text { if } n>2 \\
2 & \text { if } n=2
\end{array}>1 .\right.
$$


Using a simple scaling argument, and applying Sobolev embedding theorem to the map $V_{s_{k}}\left(D v_{k}\right)-V_{s_{k}}\left(z_{0}\right)$, we get that there exists a constant $c \equiv c(n)$ such that for any ball $B_{r} \subseteq B_{R}$

$$
\begin{aligned}
\left(f_{B_{r / 2}}\left|V_{s_{k}}\left(D v_{k}\right)-V_{s_{k}}\left(z_{0}\right)\right|^{2 \chi_{0}} d x\right)^{\frac{1}{\chi_{0}}} \leq c f_{B_{r / 2}}\left|V_{s_{k}}\left(D v_{k}\right)-V_{s_{k}}\left(z_{0}\right)\right|^{2} d x & \\
& +c r^{2} f_{B_{r / 2}}\left|D\left(V_{s_{k}}\left(D v_{k}\right)\right)\right|^{2} d x .
\end{aligned}
$$

We now use (3.1) with $V\left(D v_{0}\right) \equiv V_{s_{k}}\left(D v_{k}\right)$ for the last integral, thereby getting

$$
\left(f_{B_{r / 2}}\left|V_{s_{k}}\left(D v_{k}\right)-V_{s_{k}}\left(z_{0}\right)\right|^{2 \chi_{0}} d x\right)^{\frac{1}{\chi_{0}}} \leq c f_{B_{r}}\left|V_{s_{k}}\left(D v_{k}\right)-V_{s_{k}}\left(z_{0}\right)\right|^{2} d x .
$$

In the last two inequalities it is $c \equiv c(n, N, p, L / v)$. Inequality (3.4) for $V\left(D v_{0}\right) \equiv$ $V_{s_{k}}\left(D v_{k}\right)$ now follows from Lemma 3.1, and then Hölder's inequality again.

Step 3: Approximation. Assumptions (3.4) $)_{1,2}$ imply in a rather standard way that $a_{k}(z)$ satisfy the growth and monotonicity assumptions (2.7)-(2.8) with $s$ replaced by $s_{k}$, uniformly with respect to $k \in \mathbb{N}$. In turn this implies that $v_{k}$ is a so-called $Q$-minimum of the functional

$$
w \mapsto \int_{B_{R}}\left(|D w|^{p}+s^{p}+k^{-p}\right) d x
$$

with $Q \equiv Q(n, N, p, L / v) \geq 1$ being independent of $k \in \mathbb{N}$; for such a conclusion see Theorem 6.1 from [34] applied to the functional in (3.6), when, with the notation of [34], it is $a_{1}(x) \equiv\left[a_{2}(x)\right]^{p /(p-1)} \equiv s_{k}^{p}$. The $Q$-minimality of $v_{k}$ now easily yields

$$
\left\|D v_{k}\right\|_{L^{p}\left(B_{R}\right)} \leq c(n, N, p, L / v)\left\|\left|D v_{0}\right|+s+1\right\|_{L^{p}\left(B_{R}\right)} .
$$

Therefore, up to a non-relabeled subsequence we may assume that $\left\{v_{k}\right\}_{k}$ weakly converges to some map in $W_{0}^{1, p}\left(B_{R}\right)$; actually we may assume that $v_{k} \rightarrow v_{0}$ strongly in $W^{1, p}\left(B_{R}\right)$ too. Indeed, using that both $v_{0}$ and $v_{k}$ are solutions, and that $v_{0} \equiv v_{k}$ on $\partial B_{R}$, and making also use of (2.6), we have

$$
\begin{aligned}
\int_{B_{R}}\left|D v_{k}-D v_{0}\right|^{p} d x & \leq c \int_{B_{R}}\left\langle a_{0}\left(D v_{k}\right)-a_{0}\left(D v_{0}\right), D v_{k}-D v_{0}\right\rangle d x \\
& =c \int_{B_{R}}\left\langle a_{0}\left(D v_{k}\right)-a_{k}\left(D v_{k}\right), D v_{k}-D v_{0}\right\rangle d x \\
& \leq \frac{1}{2} \int_{B_{R}}\left|D v_{k}-D v_{0}\right|^{p} d x \\
& \quad+c \int_{B_{R}}\left|a_{0}\left(D v_{k}\right)-a_{k}\left(D v_{k}\right)\right|^{\frac{p}{p-1}} d x .
\end{aligned}
$$


The last integral tends to zero as $k \nearrow \infty$ by (3.4) 3 and (3.7). Therefore $D v_{k} \rightarrow D v_{0}$ strongly in $L^{p}\left(B_{R}\right)$, and since all the $v_{k}, v_{0}$ share the same boundary datum it follows $v_{k} \rightarrow v_{0}$ strongly in $W^{1, p}\left(B_{R}\right)$. In turn this and (2.3) imply that up to another subsequence $V_{s_{k}}\left(D v_{k}\right) \rightarrow V\left(D v_{0}\right)$ strongly in $L^{2}\left(B_{R}\right)$. Fix $z_{0} \in \mathbb{R}^{N \times n}$ as in the statement; applying estimate (3.1) to $V_{s_{k}}\left(D v_{k}\right)$ instead of $V\left(D v_{0}\right)$, which is allowed by the previous step, we infer that $\left\{V_{s_{k}}\left(D v_{k}\right)\right\}_{k}$ is bounded in $W^{1,2}\left(B_{R / 2}\right)$ and therefore it also holds $V_{s_{k}}\left(D v_{k}\right) \rightarrow V\left(D v_{0}\right)$ weakly in $W^{1,2}\left(B_{R / 2}\right)$ up to yet another subsequence. We are ready to conclude: writing estimate (3.1) with $V\left(D v_{0}\right) \equiv V_{s_{k}}\left(D v_{k}\right)$ and letting $k \nearrow \infty$ we find the final form of (3.1) for the original $V\left(D v_{0}\right)$ using strong convergence for the right-hand side, and lower semicontinuity for the left-hand one. From this last fact the inclusion $V\left(D v_{0}\right) \in W_{\text {loc }}^{1,2}(A)$ in turn follows via a covering argument. In the same way (3.2) follows from the similar inequality for the $V_{s_{k}}\left(D v_{k}\right)$ given in Step 2.

Finally, a few basic consequences of De Giorgi's regularity theory for elliptic equations, and Gehring's lemma for elliptic problems and variational integrals; see for instance [34, Chapters 6-7], for a reasonable overview of the subject.

Lemma 3.3. Let $v \in W^{1, p}(A)$ with $p \in(1, n]$ be a weak solution to the equation

$$
\operatorname{div} a(x, D v)=0 \quad \text { in } A,
$$

under the assumptions

$$
|a(x, z)| \leq c\left(s^{2}+|z|^{2}\right)^{\frac{p-1}{2}}, \quad c^{-1}|z|^{p}-c s^{p} \leq\langle a(x, z), z\rangle,
$$

for every $x \in \Omega$ and $z \in \mathbb{R}^{n}$, where $c \equiv c(L / v)$ and $\nu, L$ are the numbers given in (1.2). There exists $\beta \equiv \beta(n, p, L / v) \in(0,1]$, such that for every $q \in(0, p]$ there exists $c \equiv c(n, p, L / v, q)$ such that, whenever $B_{R} \subseteq A$, and $0<\varrho \leq R$ it holds

$$
\int_{B_{\varrho}}\left(|D v|^{q}+s^{q}\right) d x \leq c\left(\frac{\varrho}{R}\right)^{n-q+\beta q} \int_{B_{R}}\left(|D v|^{q}+s^{q}\right) d x .
$$

Moreover, there exists $\chi \equiv \chi(n, p, L / v)>1$, such that $D v \in L_{\mathrm{loc}}^{p \chi}\left(A, \mathbb{R}^{n}\right)$ and

$$
\left(f_{B_{R / 2}}|D v|^{p \chi} d x\right)^{\frac{1}{p \chi}} \leq c\left(f_{B_{R}}\left(|D v|^{q}+s^{q}\right) d x\right)^{\frac{1}{q}},
$$

where again $c \equiv c(n, p, L / v, q)$.

Proof. First observe that by (3.9) we may apply Theorem 6.1 from [34] with the choice $a_{1}(x) \equiv\left[a_{2}(x)\right]^{p /(p-1)} \equiv s^{p}$, concluding that the solution $v$ is a $Q$ minimum of the functional

$$
w \mapsto \int_{A}\left(|D w|^{p}+s^{p}\right) d x
$$


with $Q \equiv Q(n, p, L / v) \geq 1$. This in turn allows to use Theorem 6.7 from [34] that in the special case of the functional in (3.12) applies with the choice $\theta(x, u) \equiv s$, and ultimately yields the existence of higher integrability exponent $\chi \equiv \chi(n, p, L / v)>1$ such that $D v \in L_{\text {loc }}^{p \chi}\left(A, \mathbb{R}^{n}\right)$; moreover (3.11) holds for $q=p$. In turn (3.11) for every $q \in(0, p]$ follows applying Lemma 3.1 with the choice $\chi_{0} \equiv \chi$. In particular Hölder's inequality gives

$$
\left(f_{B_{R / 2}}|D v|^{p} d x\right)^{\frac{q}{p}} \leq c f_{B_{R}}\left(|D v|^{q}+s^{q}\right) d x .
$$

It remans to establish (3.10); this inequality is standard when $q=p$, that is

$$
f_{B_{\varrho}}|D v|^{p} d x \leq c\left(\frac{\varrho}{R}\right)^{-p+\beta p} f_{B_{R}}|D v|^{p} d x+c\left(\frac{\varrho}{R}\right)^{-p+\beta p} s^{p},
$$

where $\beta>0$ is as specified in the statement. For the proof of (3.14) see Remark 3.4 below. Therefore, when $\varrho \in(0, R / 2]$, using Hölder's inequality yields

$$
\begin{aligned}
f_{B_{\varrho}}|D v|^{q} d x & \leq\left(f_{B_{\varrho}}|D v|^{p} d x\right)^{\frac{q}{p}} \\
& \stackrel{(3.14)}{\leq} c\left(\frac{\varrho}{R}\right)^{-q+\beta q}\left(f_{B_{R / 2}}|D v|^{p} d x\right)^{\frac{q}{p}}+c\left(\frac{\varrho}{R}\right)^{-q+\beta q} s^{q} \\
& \stackrel{(3.13)}{\leq} c\left(\frac{\varrho}{R}\right)^{-q+\beta q} f_{B_{R}}\left(|D v|^{q}+s^{q}\right) d x .
\end{aligned}
$$

Summing $s^{q}$ to both sides of the previous inequality, taking into account that $\varrho \leq R$ and $q-\beta q \geq 0$, and finally getting rid of the averages gives (3.10) when $\varrho \in$ $(0, R / 2]$; the case $\varrho \in(R / 2, R]$ trivially follows and the lemma is completely proved.

Remark 3.4 (An esoteric detail). By carefully tracing the dependence of the constants back in De Giorgi's theory - see in particular [34], Paragraphs 7.1-7.3 - we are giving here a justification of inequality (3.14). Using Theorem 7.7 from [34], applied to the particular functional in (3.12) when $a(x) \equiv s^{p}$, and taking into account Remark 7.7 again from [34], we have that (3.14) holds in the preliminary form

$$
f_{B_{\varrho}}|D v|^{p} d x \leq c\left(\frac{\varrho}{R}\right)^{-p+\beta p} f_{B_{R}}|D v|^{p} d x+c\left\|s^{p}\right\|_{L^{t}\left(B_{R}\right)} \varrho^{-p+\beta p},
$$

where $\beta:=\min \{\tilde{\beta}(n, p, L / v), n \varepsilon / p\}$ and $\tilde{\beta}(n, p, L / v)>0,1 / t:=p / n-\varepsilon$; here $\varepsilon \in(0, p / n)$ can be picked arbitrarily small. In fact, choose $\varepsilon \equiv \varepsilon(n, p, L / v)$ small enough in order to have that $\beta=n \varepsilon / p$; then

$$
\left\|s^{p}\right\|_{L^{t}\left(B_{R}\right)} \varrho^{-p+\beta p} \leq c(n, p) s^{p} R^{p-n \varepsilon} \varrho^{-p+\beta p}=c s^{p}\left(\frac{\varrho}{R}\right)^{-p+\beta p} .
$$

Merging the latter inequality with (3.15) yields (3.14). 


\section{Comparison estimates}

Let us first introduce some notation that we shall keep for the rest of the paper; accordingly to (1.15) and (1.33), in the case $\theta \in[p, n]$ in (1.27) we define

$$
\left\{\begin{array}{lll}
\sigma(q, \theta):=\theta(1-q / m)=\theta-\frac{q(\theta-1)}{p-1} & \text { when } & p-1 \leq q<m \\
\sigma(q):=n-\frac{q(n-1)}{p-1} & \text { when } & 1 \leq q<b
\end{array}\right.
$$

Here, as in the rest of the paper, $b$ will denote the number defined in (1.9), and $m$ the one in (1.28). For the rest of the section we fix a ball

$$
B_{R} \equiv B\left(x_{0}, R\right) \subset \subset \Omega, \quad \text { with } \quad R \leq 1 .
$$

The first two lemmas are dealing with weak solutions to more regular problems i.e. $u \in W_{0}^{1, p}(\Omega)$ will be the unique solution to

$$
\begin{cases}-\operatorname{div} a(x, D u)=f \in L^{\infty}(\Omega) & \text { in } \Omega \\ u=0 & \text { on } \partial \Omega\end{cases}
$$

for a fixed $f$ to be eventually chosen; such a solution exists via standard monotonicity methods [53] as $f$ belongs to the dual of $W^{1, p}$. By the same argument we introduce $v \in u+W_{0}^{1, p}\left(B_{R}\right)$, defined as the unique solution to

$$
\begin{cases}-\operatorname{div} a(x, D v)=0 & \text { in } B_{R} \\ v=u & \text { on } \partial B_{R} .\end{cases}
$$

Lemma 4.1. Under the assumptions (1.2) with $p \leq n$, with $u \in W^{1, p}\left(B_{R}\right)$ as in (4.2), and $v \in u+W_{0}^{1, p}\left(B_{R}\right)$ as in (4.3), we have for any $1 \leq q<b$ that

$$
\int_{B_{R}}|V(D u)-V(D v)|^{2 q / p}+|D u-D v|^{q} d x \leq c\left(\int_{B_{R}}|f| d x\right)^{\frac{q}{p-1}} R^{\sigma(q)},
$$

where $\sigma(q)$ is in (4.1), and $c \equiv c(n, p, v, q)$.

Proof.

Step 1. Here we observe that we can assume $B\left(x_{0}, R\right) \equiv B_{1}$ by a scaling argument. Indeed, changing variables, we let for $y \in B_{1}$

$$
\left\{\begin{array}{l}
\tilde{u}(y):=R^{-1} u\left(x_{0}+R y\right), \tilde{v}(y):=R^{-1} v\left(x_{0}+R y\right), \\
\tilde{a}(y, z):=a\left(x_{0}+R y, z\right), \tilde{f}(y):=R f\left(x_{0}+R y\right), \quad B_{R} \equiv B\left(x_{0}, R\right), \\
-\operatorname{div} \tilde{a}(y, D \tilde{u})=\tilde{f}, \quad-\operatorname{div} \tilde{a}(y, D \tilde{v})=0 .
\end{array}\right.
$$


Obviously $\tilde{u} \equiv \tilde{v}$ on $\partial B_{1}$. It is readily verified that the new vector field $\tilde{a}$ still satisfies (1.2). Now (4.4) follows by writing its corresponding version for $R=1$, and scaling back to $B_{R}$.

Step 2. Here we prove the following implication:

$$
\int_{B_{1}}|f| d x \leq 1 \Longrightarrow \int_{B_{1}}|V(D u)-V(D v)|^{2 q / p}+|D u-D v|^{q} d x \leq c_{2},
$$

with $c_{2} \equiv c_{2}(n, p, v, q)$. Notice that the following computations remain valid also for $q \in[1, p-1)$. In order to prove (4.6) we shall revisit the technique of [8], reporting the necessary modifications in some detail for the sake of clarity. For $k>0$, let us define the following truncation operators, classical after [8]:

$$
T_{k}(s):=\max \{-k, \min \{k, s\}\}, \quad \Phi_{k}(s):=T_{1}\left(s-T_{k}(s)\right), \quad s \in \mathbb{R} .
$$

Since both $u$ and $v$ are solutions, we test the weak formulation

$$
\int_{B_{1}}\langle a(x, D u)-a(x, D v), D \varphi\rangle d x=\int_{B_{1}} f \varphi d x
$$

with $\varphi \equiv T_{k}(u-v)$; this function is admissible as it is in $L^{\infty}\left(B_{R}\right) \cap W_{0}^{1, p}\left(B_{R}\right)$, and we have (2.8). Using the monotonicity inequalities (2.5)-(2.6), and the bound in (4.6), we easily obtain with $c \equiv c(n, p, v)$

$$
\int_{D_{k}}|V(D u)-V(D v)|^{2}+|D u-D v|^{p} d x \leq c k \int_{B_{1}}|f| d x \leq c k .
$$

Here we have set

$$
D_{k}:=\left\{x \in B_{1}:|u(x)-v(x)| \leq k\right\} .
$$

Moreover, testing again (4.8) with $\varphi \equiv \Phi_{k}(u-v)$, and again using (2.5)-(2.6) and the bound in (4.6), we obtain

$$
\int_{C_{k}}|V(D u)-V(D v)|^{2}+|D u-D v|^{p} d x \leq c \int_{B_{1}}|f| d x \stackrel{(4.6)}{\leq} c,
$$

where this time

$$
C_{k}:=\left\{x \in B_{1}: k<|u(x)-v(x)| \leq k+1\right\},
$$

and $c \equiv c(n, p, v)$. By Hölder's inequality, and the very definition of $C_{k}$, we find

$$
\begin{aligned}
\int_{C_{k}}|V(D u)-V(D v)|^{2 q / p}+|D u-D v|^{q} d x \\
\leq c\left|C_{k}\right|^{1-\frac{q}{p}}\left(\int_{C_{k}}|V(D u)-V(D v)|^{2}+|D u-D v|^{p} d x\right)^{\frac{q}{p}} \\
\quad \stackrel{(4.11)}{\leq} c\left|C_{k}\right|^{1-\frac{q}{p}} \leq \frac{c}{k^{q^{*}\left(1-\frac{q}{p}\right)}}\left(\int_{C_{k}}|u-v|^{q^{*}} d x\right)^{1-\frac{q}{p}} .
\end{aligned}
$$


With $0 \neq k_{0} \in \mathbb{N}$ to be fixed later, we have, using the previous inequality and Hölder's inequality for sequences, and finally Sobolev's embedding theorem, as $q<n$ under the present assumptions:

$$
\begin{gathered}
\int_{B_{1}}|V(D u)-V(D v)|^{2 q / p}+|D u-D v|^{q} d x \\
=\int_{D_{k_{0}}}|V(D u)-V(D v)|^{2 q / p}+|D u-D v|^{q} d x \\
+\sum_{k=k_{0}}^{\infty} \int_{C_{k}}|V(D u)-V(D v)|^{2 q / p}+|D u-D v|^{q} d x \\
\stackrel{(4.9)}{\leq} c k_{0}+c\left[\sum_{k=k_{0}}^{\infty} \frac{1}{k^{q^{*}\left(\frac{p-q}{q}\right)}}\right]^{\frac{q}{p}}\left(\int_{B_{1}}|u-v|^{q^{*}} d x\right)^{1-\frac{q}{p}} \\
\leq c k_{0}+c H\left(k_{0}\right)\left(\int_{B_{1}}|D u-D v|^{q} d x\right)^{\frac{q^{*}}{q}\left(1-\frac{q}{p}\right)},
\end{gathered}
$$

with

$$
H\left(k_{0}\right):=\left[\sum_{k=k_{0}}^{\infty} \frac{1}{k^{q^{*}\left(\frac{p-q}{q}\right)}}\right]^{\frac{q}{p}}, \quad \text { and } \quad c \equiv c(n, p, v, q) .
$$

Here $H\left(k_{0}\right)$ is finite since $q<b$ implies that $q^{*}(p / q-1)>1$. We finally distinguish two cases. If $p<n$ then we take $k_{0}=1$ in (4.14), and observe that $\gamma:=\left(q^{*} / q\right)(1-q / p)<1$. Therefore, applying Young's inequality in (4.14) with conjugate exponents $1 / \gamma$ and $1 /(1-\gamma)$ we find (4.6). In the case $p=n$ we have that $\gamma=1$ and the previous argument does not work; instead, we choose $k_{0}$ large enough in order to have $c H\left(k_{0}\right)=1 / 2$ in (4.14), and (4.6) follows again. Observe that this determines $k_{0} \equiv k_{0}(n, p, v, q)$ possibly large, and this finally reflects in the constant $c$ appearing in (4.6).

Step 3. We are ready to conclude the whole proof, again by mean of a scaling argument. We shall prove the validity of the estimate for $B_{R} \equiv B_{1}$, and then we shall conclude using Step 1 . Without loss of generality we assume that $A:=$ $\|f\|_{L^{1}\left(B_{1}\right)}^{1 /(p-1)}>0$, otherwise $u \equiv v$ and the assertion is trivially verified. We define the new solutions $\tilde{u}:=A^{-1} u, \tilde{v}:=A^{-1} v$, the new datum $\tilde{f}:=A^{1-p} f$, and the new vector field $\tilde{a}(x, z):=A^{1-p} a(x, A z)$. Therefore we have that $\tilde{u} \equiv \tilde{v}$ on $\partial B_{1}$, and moreover $\operatorname{div} \tilde{a}(x, D \tilde{u})=\tilde{f}, \operatorname{div} \tilde{a}(x, D \tilde{v})=0$, in the weak sense. We make sure that we can apply the result in Step 2 . Trivially $\|\tilde{f}\|_{L^{1}\left(B_{1}\right)}=1$ and moreover it is easy to see that the vector field $\tilde{a}(x, z)$ satisfies (1.2) with $s$ replaced by $s / A \geq 0$. Therefore the inequality in (4.6) holds in the form

$$
\int_{B_{1}}\left|V_{S / A}(D \tilde{u})-V_{s / A}(D \tilde{v})\right|^{2 q / p}+|D \tilde{u}-D \tilde{v}|^{q} d x \leq c_{2}, \quad c_{2} \equiv c_{2}(n, p, v, q) .
$$


Re-scaling back from $\tilde{u}-\tilde{v}$ to $u-v$ and using (2.4), we find

$$
\int_{B_{1}}|V(D u)-V(D v)|^{2 q / p}+|D u-D v|^{q} d x \leq c_{2}\left(\int_{B_{1}}|f| d x\right)^{\frac{q}{p-1}}
$$

and the proof is concluded via Step 1.

Remark 4.2. Although the previous lemma has been stated for $q \geq 1$ we shall use it only for the case $q \geq p-1$.

Lemma 4.3. Under the assumptions (1.2) with $p \leq n$, assume $p-1 \leq q<b$, and $f \in L^{1, \theta}\left(B_{R}\right)$. With $u \in W_{0}^{1, p}\left(B_{R}\right)$ as in (4.2), and $v \in u+W_{0}^{1, p}\left(B_{R}\right)$ as in (4.3), we have for any $R \leq 1$

$$
\int_{B_{R}}|V(D u)-V(D v)|^{\frac{2 q}{p}}+|D u-D v|^{q} d x \leq c\|f\|_{L^{1, \theta\left(B_{R}\right)}}^{\frac{q-p+1}{p-1}} \int_{B_{R}}|f| d x R^{\sigma(q, \theta)}
$$

where $\sigma(q, \theta)$ is in (4.1), and $c \equiv c(n, p, v, q)$.

Proof. First observe that the definition in (4.1) implies

$$
(n-\theta)\left(\frac{q}{p-1}-1\right)+\sigma(q, n)=\sigma(q, \theta) .
$$

Now, since $p-1 \leq q$ we may estimate

$$
\left(\int_{B_{R}}|f| d x\right)^{\frac{q}{p-1}} \leq R^{(n-\theta)\left(\frac{q}{p-1}-1\right)}\|f\|_{L^{1, \theta}\left(B_{R}\right)}^{\frac{q-p+1}{p-1}} \int_{B_{R}}|f| d x
$$

and then we conclude by merging (4.17) with (4.4), taking (4.16) into account.

The next twin lemmata are about the capacitary case $\theta<p$. In the following $u$ will be the solution to (1.1), and $\mu$ the Radon measure in (1.1) $)_{1}$. We have $u \in W_{0}^{1, p}(\Omega)$, and $u$ is the unique solution, since under the assumptions considered in the next two lemmata it is $\mu \in W^{-1, p^{\prime}}(\Omega)$ by a theorem of D. R. Adams [1,3].

Lemma 4.4. Under the assumptions (1.2) with $p>n$, and with $u, v \in W^{1, p}\left(B_{R}\right)$ as in (1.1) and (4.3) respectively, if (1.27) holds then

$$
\int_{B_{R}}|V(D u)-V(D v)|^{2} d x \leq c M^{\frac{1}{p-1}}|\mu|\left(B_{R}\right) R^{\sigma(p)}
$$

where $\sigma(p)=(p-\theta) /(p-1)$ is as in (1.36), and $c \equiv c(n, p, v)$. 
Proof. Notice that here it can be also $\theta=n$. We test the weak formulation

$$
\int_{B_{R}}\langle a(x, D u)-a(x, D v), D \varphi\rangle d x=\int_{B_{R}} \varphi d \mu,
$$

with $\varphi \equiv u-v$, which is admissible as $p>n$ and therefore both $u$ and $v$ are Hölder continuous. Moreover, using Morrey-Sobolev's embedding theorem, and the fact that $u \equiv v$ on $\partial B_{R}$, we estimate

$$
\begin{aligned}
\left|\int_{B_{R}}(u-v) d \mu\right| & \leq\|u-v\|_{L^{\infty}\left(B_{R}\right)}|\mu|\left(B_{R}\right) \\
& \leq c R^{\frac{p-n}{p}}\|D u-D v\|_{L^{p}\left(B_{R}\right)}|\mu|\left(B_{R}\right) .
\end{aligned}
$$

Combining the last inequality with (4.19) and using (2.5)-(2.6) we gain

$$
\|V(D u)-V(D v)\|_{L^{2}\left(B_{R}\right)}^{2}+\|D u-D v\|_{L^{p}\left(B_{R}\right)}^{p} \leq c R^{\frac{p-n}{p}}\|D u-D v\|_{L^{p}\left(B_{R}\right)}|\mu|\left(B_{R}\right),
$$

thereby, applying Young's inequality and then using (1.27) we conclude

$$
\begin{aligned}
& \|V(D u)-V(D v)\|_{L^{2}\left(B_{R}\right)}^{2}+\|D u-D v\|_{L^{p}\left(B_{R}\right)}^{p} \\
& \leq c R^{\frac{p-n}{p-1}}\left[|\mu|\left(B_{R}\right)\right]^{\frac{p}{p-1}} \leq c R^{\frac{p-\theta}{p-1}} M^{\frac{1}{p-1}}|\mu|\left(B_{R}\right) .
\end{aligned}
$$

Lemma 4.5. Under the assumptions (1.2) and (1.27) with $0 \leq \theta<p<n$, and with $u, v \in W^{1, p}\left(B_{R}\right)$ as in (1.1) and (4.3) respectively, we have that (4.18) holds, with $\sigma(p)$ as in (1.36) and $c \equiv c(n, p, v, \theta)$. In the case $0 \leq \theta<p=n$ estimate (4.18) remains valid modulo replacing the right-hand side by $c M^{1 /(n-1)}|\mu|\left(B_{R}\right) R^{\sigma}$, for any choice $\sigma<\sigma(n)=(n-\theta) /(n-1)$, where $c \equiv c(n, v, \theta, \sigma)$.

Proof. Firstly we deal with the case $p<n$. We test (4.19) with $\varphi \equiv u-v$, which is again admissible since $\theta<p$ implies that $\mu \in W^{-1, p^{\prime}}(\Omega)$. Therefore using again monotonicity (2.6) as for the previous lemma, using Hölder's inequality and applying Theorem 2.9 with the measure $\lambda \equiv|\mu|$, we have

$$
\begin{gathered}
\|V(D u)-V(D v)\|_{L^{2}\left(B_{R}\right)}^{2}+\|D u-D v\|_{L^{p}\left(B_{R}\right)}^{p} \leq c\left|\int_{B_{R}}(u-v) d \mu\right| \\
\leq c\left[|\mu|\left(B_{R}\right)\right]^{1-\frac{1}{p}}\left(\int_{B_{R}}|u-v|^{p} d|\mu|\right)^{\frac{1}{p}} \\
\leq c M^{\frac{1}{p}} R^{\frac{p-\theta}{p}}\left[|\mu|\left(B_{R}\right)\right]^{1-\frac{1}{p}}\left(\int_{B_{R}}|D u-D v|^{p} d x\right)^{\frac{1}{p}} .
\end{gathered}
$$

Using again Young's inequality yields (4.18). In order to treat the case $p=n$ it suffices to use Theorem 2.9 again, and applying it in (4.20) as for the case $p<n$. 
Finally, a comparison lemma of a different type. With $B_{\bar{R}} \equiv B\left(x_{0}, \bar{R}\right) \subseteq$ $B\left(x_{0}, R\right)$ and $v$ as in (4.3), let us define $v_{0} \in v+W_{0}^{1, p}\left(B_{\bar{R}}\right)$ as the unique weak solution to

$$
\begin{cases}-\operatorname{div} a\left(x_{0}, D v_{0}\right)=0 & \text { in } B_{\bar{R}} \\ v_{0}=v & \text { on } \partial B_{\bar{R}}\end{cases}
$$

Lemma 4.6. Under the assumptions (1.2) and (1.4), with $v$ as in (4.3) and $v_{0}$ as in (4.21), with $c \equiv c(n, p, L / v)$ we have

$$
\int_{B_{\bar{R}}}\left|V\left(D v_{0}\right)-V(D v)\right|^{2} d x \leq c \bar{R}^{2} \int_{B_{\bar{R}}}\left(|D v|^{p}+s^{p}\right) d x .
$$

Proof. Using (2.7)-(2.8) it follows that $v_{0}$ is a $Q$-minimum of the functional $w \mapsto$ $\int_{B_{R}}\left(|D w|^{p}+s^{p}\right) d x$, with $Q \equiv Q(n, p, L / v)$, see Theorem 6.1 from [34] that implies

$$
\int_{B_{\bar{R}}}\left|D v_{0}\right|^{p} d x \leq c(n, p, L / v) \int_{B_{\bar{R}}}\left(|D v|^{p}+s^{p}\right) d x .
$$

In turn, using (1.2) $)_{1}$, and the fact that both $v$ and $v_{0}$ are solutions, we have

$$
\begin{aligned}
\int_{B_{\bar{R}}} & \left(s^{2}+\left|D v_{0}\right|^{2}+|D v|^{2}\right)^{\frac{p-2}{2}}\left|D v-D v_{0}\right|^{2} d x \\
& \leq c \int_{B_{\bar{R}}}\left\langle a\left(x_{0}, D v\right)-a\left(x_{0}, D v_{0}\right), D v-D v_{0}\right\rangle d x \\
& =c \int_{B_{\bar{R}}}\left\langle a\left(x_{0}, D v\right)-a(x, D v), D v-D v_{0}\right\rangle d x \\
& (1.4) \operatorname{s} \int_{B_{\bar{R}}}\left(s^{2}+\left|D v_{0}\right|^{2}+|D v|^{2}\right)^{\frac{p-1}{2}}\left|D v-D v_{0}\right| d x \\
& \leq \frac{1}{2} \int_{B_{\bar{R}}}\left(s^{2}+\left|D v_{0}\right|^{2}+|D v|^{2}\right)^{\frac{p-2}{2}}\left|D v-D v_{0}\right|^{2} d x \\
& +c \bar{R}^{2} \int_{B_{\bar{R}}}\left(s^{2}+\left|D v_{0}\right|^{2}+|D v|^{2}\right)^{\frac{p}{2}} d x .
\end{aligned}
$$

Using (2.2) for the left-hand side, we get

$$
\int_{B_{\bar{R}}}\left|V\left(D v_{0}\right)-V(D v)\right|^{2} d x \leq c \bar{R}^{2} \int_{B_{\bar{R}}}\left(|D v|^{p}+\left|D v_{0}\right|^{p}+s^{p}\right) d x,
$$

and (4.22) follows by merging the latter inequality with (4.23).

Remark 4.7 (Global estimates by scaling). We consider (4.2), and we find a global a priori estimate for $u$, making explicit the ones in [8]. Let us go back to Lemma 4.1, Step 2, and let's modify a bit the estimates given there. Assume not 
only that $\|f\|_{L^{1}(\Omega)} \leq 1$ as in (4.6), but now also that $s^{q}|\Omega| \leq 1$. Just forget about $v$, and test $(4.2)_{1}$ on the whole $\Omega$ with $T_{k}(u)$ and $\Phi_{k}(u)$, as $u \in W_{0}^{1, p}(\Omega)$; then use (2.7) to get

$$
\left\{\begin{array}{l}
\int_{D_{k}}|D u|^{p} d x \leq c k\|f\|_{L^{1}(\Omega)}+c s^{p}\left|D_{k}\right| \\
\int_{C_{k}}|D u|^{p} d x \leq c\|f\|_{L^{1}(\Omega)}+c s^{p}\left|C_{k}\right|,
\end{array}\right.
$$

where this time $D_{k}:=\{x \in \Omega:|u(x)| \leq k\}$ and $C_{k}:=\{x \in \Omega: k<|u(x)| \leq$ $k+1\}$, consistently with (4.10) and (4.12) respectively, and the constant $c$ just depends on $n, p, L / v$. Then proceed as in (4.13), but using (4.24), and we get

$$
\|D u\|_{L^{q}\left(C_{k}\right)}^{q} \leq c\left|C_{k}\right|^{1-q / p}+c s^{q}\left|C_{k}\right| .
$$

Summing up these inequalities as for (4.14), the terms $\left|C_{k}\right|^{1-q / p}$ are treated as in (4.13) and subsequent estimates, while, obviously, $s^{q}\left|D_{k_{0}}\right|+\sum_{k \geq k_{0}} s^{q}\left|C_{k}\right|=$ ${ }_{s}^{q}|\Omega|$. Therefore, when $p<n$, it follows that $\|D u\|_{L^{q}(\Omega)}^{q} \leq c\left(1+s^{q}|\Omega|\right) \leq \tilde{c}$, where $\tilde{c}$ is universal in the sense it only depends on $n, p, L / v, q$, and on $\Omega$. In the case $p=n$, which already requires a different treatment in Lemma 4.1, $\tilde{c}$ must be replaced by $\tilde{c}\left(|\Omega|^{1 / q-1 / p}+1\right)$; indeed we need to use also

$$
\|D u\|_{L^{q}\left(D_{k_{0}}\right)}^{q} \leq c\left(k_{0}^{q / p}\|f\|_{L^{1}(\Omega)}^{q / p}|\Omega|^{1-q / p}+s^{q}|\Omega|\right) \leq \tilde{c}\left(|\Omega|^{1-q / p}+1\right),
$$

that comes from (4.24) exactly as in Lemma 4.1, Step 2, case $p=n$. Now we use a scaling argument to treat the general case. Define, $A:=\|f\|_{L^{1}(\Omega)}^{1 /(p-1)}+s|\Omega|^{1 / q}>0$, and accordingly, $\tilde{u}:=A^{-1} u, \bar{f}:=A^{1-p} f, \tilde{a}(x, z):=A^{1-p} a(x, A z)$, so that the vector field $\tilde{a}$ satisfies (1.2) with $s$ replaced by $s / A$. Moreover $\tilde{u}$ satisfies div $\tilde{a}(x, \tilde{u})=\bar{f}$ in the weak sense and obviously $\tilde{u} \in W_{0}^{1, p}(\Omega)$. By the definition of $A$ we have that $\|\tilde{f}\|_{L^{1}(\Omega)} \leq 1$ and $(s / A)^{q}|\Omega| \leq 1$, therefore we get the universal bounds $\|D \tilde{u}\|_{L^{q}(\Omega)} \leq \tilde{c}$ when $p<n$, and $\|D \tilde{u}\|_{L^{q}(\Omega)} \leq \tilde{c}\left(|\Omega|^{1 / q-1 / n}+1\right)$ when $p=n$. Taking into account the definitions of $\tilde{a}$ and $A$ the latter inequalities readily give

$$
\|D u\|_{L^{q}(\Omega)} \leq c\|f\|_{L^{1}(\Omega)}^{1 /(p-1)}+c s|\Omega|^{1 / q},
$$

that is the estimate we were looking for; the constant $c$ in (4.25) will depend on $n, p, q, L / v$, and $\Omega$. The dependence on $\Omega$ is on $\left(|\Omega|^{1 / q-1 / n}+1\right)$ in the case $p=n$. As for Step 2 from Lemma 4.1, here everything works for $q \in[1, p-1)$ too.

Remark 4.8. An a priori estimate can be derived for the super-capacitary case of Theorem 1.10 too. Testing (1.7) with $u$, and this is possible since $\theta<p$ implies $\mu \in W^{-1, p^{\prime}}$, using (2.7), and proceeding as in Lemmata 4.4 and 4.5 , but using (2.23) instead of (2.21), we have, with $c$ as in Theorem 1.10

$$
\|D u\|_{L^{p}(\Omega)}^{p} \leq c M^{1 /(p-1)}|\mu|(\Omega)+c s^{p}|\Omega| .
$$




\section{Basic approximation}

In order to establish the existence and regularity results for the problem (1.1) for a general measure $\mu$, a standard device $[8,26]$ is to consider solutions to suitable approximate problems, and then to prove a priori estimates; the main feature of such solutions is to be in the natural space $W_{0}^{1, p}(\Omega)$. Then the final assertion follows by a suitable passage-to-the-limit argument. We remark that this procedure is not necessary when considering the assumptions of Theorem 1.10, that is when $\theta<$ $p$ ( $p$-capacitary measures). In this section we set up the approximation scheme, considered in the rest of the paper for the case $\theta \in[p, n]$. In fact, as already remarked in the Introduction, in the case $\theta<p$ the measure $\mu$ belongs to the dual space $W^{-1, p^{\prime}}$ (see [1]), and at this point the standard monotone operator theory provides a unique solution to (1.1) in the natural energy space $W_{0}^{1, p}(\Omega)$, therefore no approximation with $W^{1, p}$-solutions is obviously needed.

We consider a standard, symmetric and non-negative mollifier $\phi \in C_{0}^{\infty}\left(B_{1}\right)$ such that $\|\phi\|_{L^{1}\left(\mathbb{R}^{n}\right)}=1$, and then define, for $k \in \mathbb{N}, \phi_{k}(x):=k^{n} \phi(k x)$. Finally the functions $f_{k}: \mathbb{R}^{n} \rightarrow \mathbb{R}$ are defined via convolution, $f_{k}(x):=\left(\mu * \phi_{k}\right)(x)$. Since in particular $f_{k} \in L^{\infty}(\Omega)$, applying standard monotonicity methods [53] we can find a unique $u_{k} \in W_{0}^{1, p}(\Omega)$ such that

$$
\begin{cases}-\operatorname{div} a\left(x, D u_{k}\right)=f_{k} & \text { in } \Omega \\ u_{k}=0 & \text { on } \partial \Omega\end{cases}
$$

From now on and for the rest of the paper the sequence $\left\{u_{k}\right\}_{k} \subset W_{0}^{1, p}(\Omega)$ will be the one fixed by (5.1). Let us collect some basic facts now. Up to extracting a non-relabeled subsequence we can assume

$$
f_{k} \rightarrow \mu \quad \text { weakly in the sense of measures. }
$$

Moreover, looking at [62], Proposition 2.7, we have

$$
\left\|f_{k}\right\|_{L^{1}(\Omega)} \leq|\mu|(\Omega), \quad\left\|f_{k}\right\|_{L^{1, \theta}(\Omega)} \leq\|\mu\|_{L^{1, \theta}(\Omega)} \stackrel{(1.27)}{\leq} M,
$$

and

$$
\left\|f_{k}\right\|_{L^{1}\left(B_{R}\right)} \leq|\mu|\left(B_{R+1 / k}\right), \quad\left\|f_{k}\right\|_{L^{1, \theta}\left(B_{R}\right)} \leq\|\mu\|_{L^{1, \theta}\left(B_{R+1 / k}\right)} .
$$

Applying Remark 4.7 and in particular estimate (4.25) to $u_{k}$, and eventually using (2.3), we get

$$
\int_{\Omega}\left|V\left(D u_{k}\right)\right|^{2 q / p} d x+\int_{\Omega}\left|D u_{k}\right|^{q} d x \leq c[|\mu|(\Omega)]^{\frac{q}{p-1}}+c s^{q}|\Omega|, \quad \forall q<b,
$$

where $c \equiv c(n, p, L / v, q, \Omega)$, and is independent of $k \in \mathbb{N}$. Therefore we immediately obtain that up to a non-relabeled subsequence

$$
u_{k} \rightarrow u \text { weakly in } W^{1, q}(\Omega) \quad \text { and } \quad u_{k} \rightarrow u \text { strongly in } L^{q}(\Omega) .
$$


The function $u$ is eventually shown to be a solution of (1.1). The proof of this fact usually involves certain truncation arguments $[8,9]$ to prove the strong convergence of the gradients. Thanks to the stronger a priori estimates we derive here we shall give a very short proof of such convergence; see Theorem 1.2. The function $u$ is the solution the Theorems and results of Section 1 in the super-capacitary case $\theta \geq p$.

\section{General measures}

This section is mainly devoted to the proof of Theorems 1.2-1.4 and Corollary 1.5. The ingredients will be: the lemmata of Section 4, the key estimate below the growth exponent (3.2), and a variant of a fractional regularity technique recently introduced in [48] in order to obtain singular sets estimates for variational problems.

Warning. In the rest of the paper we shall very often deal with a solution $u$ to problem (4.2), for a fixed, but a priori un-specified $L^{\infty}$ function $f$. Eventually we shall take $f \equiv f_{k}$ and $u \equiv u_{k}$, where $u_{k}, f_{k}$ appear in (5.1).

Keeping (4.1) in mind, let us define

$$
\delta:=\frac{p \sigma(q, \theta)}{2 q} ; \quad \gamma(t):=\frac{\delta}{\delta+1-t}, \quad \text { for every } t \in[0, \delta+1) .
$$

Remark 6.1. We have $\delta \leq 1$. Indeed, by (4.1), when $p-1 \leq q$ then $\sigma(q, \theta) \leq 1$, therefore

$$
\delta \leq \frac{p}{2 q} \leq \frac{p}{2(p-1)} \leq 1,
$$

which holds since $p \geq 2$. For the same reason we have $2 q / p \geq 1$ for $q \geq p-1$.

We shall start deriving a priori estimates for $W^{1, p}$-solutions to (4.2). We set

$$
\bar{f}:=\|f\|_{L^{1, \theta}\left(\Omega^{\prime \prime}\right)}^{\frac{q-p+1}{p-1}} f \quad \text { if } \quad p-1 \leq q
$$

where $\Omega^{\prime \prime} \subseteq \Omega$ will be clarified in Lemmas 6.2 and 6.3 below. When $\theta=n$ it follows directly from definition (2.14) that

$$
\|\bar{f}\|_{L^{1}(\Omega)} \leq\|f\|_{L^{1}(\Omega)}^{\frac{q}{p-1}} .
$$

Lemma 6.2. Let $u \in W_{0}^{1, p}(\Omega)$ be the unique solution to (4.2), under the assumptions (1.2) and (1.4) with $p \leq n$, and let $q$ be such that $p-1 \leq q<b$. Assume that

$$
V(D u) \in W_{\mathrm{loc}}^{t, 2 q / p}\left(\Omega, \mathbb{R}^{n}\right), \quad \text { for some } t \in[0, \delta),
$$


where $\delta$ is as in (6.1), and that for every couple of open subsets $\Omega^{\prime} \subset \subset \Omega^{\prime \prime} \subset \subset \Omega$ there exists $c_{1} \equiv c_{1}\left(\operatorname{dist}\left(\Omega^{\prime}, \partial \Omega^{\prime \prime}\right)\right)$, such that

$$
[V(D u)]_{t, 2 q / p ; \Omega^{\prime}}^{2 q / p} \leq c_{1} \int_{\Omega^{\prime \prime}}\left(|D u|^{q}+s^{q}+|\bar{f}|\right) d x .
$$

Then

$$
V(D u) \in W_{\mathrm{loc}}^{\tilde{t}, 2 q / p}\left(\Omega, \mathbb{R}^{n}\right), \quad \text { for every } \tilde{t} \in[0, \gamma(t)),
$$

where $\gamma(\cdot)$ is in (6.1), and for every couple of open subsets $\Omega^{\prime} \subset \subset \Omega^{\prime \prime} \subset \subset \Omega$ there exists a new constant $c$ depending only on $n, p, L / v, q$, $\operatorname{dist}\left(\Omega^{\prime}, \partial \Omega^{\prime \prime}\right), \tilde{t}, c_{1}$, such that

$$
[V(D u)]_{\tilde{t}, 2 q / p ; \Omega^{\prime}}^{2 q / p} \leq c \int_{\Omega^{\prime \prime}}\left(|D u|^{q}+s^{q}+|\bar{f}|\right) d x .
$$

Moreover, for every $i \in\{1, \ldots, n\}$ and with $0<|h|<\operatorname{dist}\left(\Omega^{\prime}, \partial \Omega^{\prime \prime}\right)$

$$
\sup _{h} \int_{\Omega^{\prime}} \frac{\left|\tau_{i, h} V(D u(x))\right|^{2 q / p}}{|h|^{\gamma(t) 2 q / p}} d x \leq c \int_{\Omega^{\prime \prime}}\left(|D u|^{q}+s^{q}+|\bar{f}|\right) d x .
$$

Proof. We fix a notation that we shall keep for the rest of the paper. Let us take $B \subset \subset \Omega$, a ball of radius $R$; we shall denote by $Q_{\text {inn }} \equiv Q_{\text {inn }}(B)$ and $Q_{\text {out }} \equiv$ $Q_{\text {out }}(B)$ the largest and the smallest cubes, concentric to $B$ and with sides parallel to the coordinate axes, contained in $B$ and containing $B$, respectively; clearly $|B| \approx$ $\left|Q_{\text {inn }}\right| \approx\left|Q_{\text {out }}\right| \approx R^{n}$. The cubes $Q_{\text {inn }}(B)$ and $Q_{\text {out }}(B)$ will be called the inner and the outer cubes of $B$, respectively. We also denote the enlarged ball as $\hat{B} \equiv 16 B$. Consistently with such a notation we put $Q_{\text {inn }} \equiv Q_{\text {inn }}(B)$ and $\hat{Q}_{\text {out }} \equiv Q_{\text {out }}(\hat{B})$, and therefore we have the following chain of inclusions:

$$
Q_{\text {inn }} \subset B \subset \subset 2 B \subset \subset 4 B \subset Q_{\text {inn }}(\hat{B}) \subset \hat{B} \subset \hat{Q}_{\text {out }}
$$

Now we fix arbitrary open subsets $\Omega^{\prime} \subset \subset \Omega^{\prime \prime} \subset \subset \Omega$, and then take $\beta \in(0,1)$ to be chosen later, and let $h \in \mathbb{R}$ be a real number satisfying

$0<|h| \leq \min \left\{\left(\frac{\operatorname{dist}\left(\Omega^{\prime}, \partial \Omega^{\prime \prime}\right)}{10000 \sqrt{n}}\right)^{\frac{1}{\beta}},\left(\frac{1}{10000}\right)^{\frac{1}{1-\beta}}\right\}=: d<\operatorname{dist}\left(\Omega^{\prime}, \partial \Omega^{\prime \prime}\right)$.

We take $x_{0} \in \Omega^{\prime}$, and fix a ball of radius $|h|^{\beta}$

$$
B \equiv B(h)=B\left(x_{0},|h|^{\beta}\right) .
$$

By (6.10) we have $\hat{Q}_{\text {out }} \subset \Omega^{\prime \prime}$. Let us first define $v \in u+W_{0}^{1, p}(\hat{B})$, and then $v_{0} \in v+W_{0}^{1, p}(8 B)$, as the unique solutions to the following Dirichlet problems:

$$
\begin{cases}-\operatorname{div} a(x, D v)=0 & \text { in } \hat{B} \\ v=u & \text { on } \partial \hat{B}\end{cases}
$$


and

$$
\begin{cases}-\operatorname{div} a\left(x_{0}, D v_{0}\right)=0 & \text { in } 8 B \\ v_{0}=v & \text { on } \partial 8 B\end{cases}
$$

respectively. Now we fix $i \in\{1, \ldots, n\}$ and write, using that $|h| \leq d$ from (6.10)

$$
\begin{aligned}
\int_{B}\left|\tau_{i, h} V(D u)\right|^{2 q / p} d x \leq & c \int_{B}\left|\tau_{i, h} V\left(D v_{0}\right)\right|^{2 q / p} d x \\
& +c \int_{B}\left|V\left(D u\left(x+h e_{i}\right)\right)-V\left(D v\left(x+h e_{i}\right)\right)\right|^{2 q / p} d x \\
& +c \int_{B}\left|V\left(D v\left(x+h e_{i}\right)\right)-V\left(D v_{0}\left(x+h e_{i}\right)\right)\right|^{2 q / p} d x \\
& +c \int_{B}|V(D u)-V(D v)|^{2 q / p} d x \\
& +c \int_{B}\left|V(D v)-V\left(D v_{0}\right)\right|^{2 q / p} d x \\
\leq & c \int_{B}\left|\tau_{i, h} V\left(D v_{0}\right)\right|^{2 q / p} d x \\
& +c \int_{\hat{B}}|V(D u)-V(D v)|^{2 q / p} d x \\
& +c \int_{2 B}\left|V(D v)-V\left(D v_{0}\right)\right|^{2 q / p} d x \\
= & I+I I+I I I .14)
\end{aligned}
$$

In order to estimate $I I$ we shall use Lemmas 4.1 and 4.3 , this last one when $q \geq$ $p-1$ and $\theta<n$; by the definition of $\sigma(q, \theta)$ in (4.1), we have

$$
\int_{\hat{B}}|V(D u)-V(D v)|^{2 q / p} d x \leq c\left(\int_{\hat{B}}|\bar{f}| d x\right)|h|^{\beta \sigma(q, \theta)},
$$

where we used (6.2) too. To estimate I I I we first appeal to Lemma 4.6 that gives

$$
\int_{8 B}\left|V\left(D v_{0}\right)-V(D v)\right|^{2} d x \leq c\left(\int_{8 B}\left(s^{2}+|D v|^{2}\right)^{\frac{p}{2}} d x\right)|h|^{\beta 2},
$$

and then apply Lemma 3.3 to $v$ in (6.12); with $\chi \equiv \chi(n, p, L / \nu)>1$ being the 
exponent determined in in Lemma 3.3 we have

$$
\begin{aligned}
& \int_{8 B}\left|V\left(D v_{0}\right)-V(D v)\right|^{2 q / p} d x \leq c|h|^{n \beta\left(1-\frac{q}{p}\right)}\left(\int_{8 B}\left|V\left(D v_{0}\right)-V(D v)\right|^{2} d x\right)^{\frac{q}{p}} \\
& \stackrel{(6.16)}{\leq} c|h|^{\frac{\beta 2 q}{p}+n \beta}\left(f_{8 B}\left(s^{2}+|D v|^{2}\right)^{\frac{p}{2}} d x\right)^{\frac{q}{p}} \\
& \leq c|h|^{\frac{\beta 2 q}{p}+n \beta}\left(f_{8 B}\left(s^{2}+|D v|^{2}\right)^{\frac{p \chi}{2}} d x\right)^{\frac{q}{p \chi}} \\
& \stackrel{(3.11)}{\leq} c|h|^{\frac{\beta 2 q}{p}} \int_{\hat{B}}\left(s^{2}+|D v|^{2}\right)^{\frac{q}{2}} d x \\
& \stackrel{(2.3)}{\leq} c|h|^{\frac{\beta 2 q}{p}} \int_{\hat{B}}\left(s^{2}+|V(D v)|^{\frac{4}{p}}\right)^{\frac{q}{2}} d x \\
& \stackrel{(2.3)}{\leq} c|h|^{\frac{\beta 2 q}{p}} \int_{\hat{B}}\left(s^{q}+|D u|^{q}\right. \\
& \left.+|V(D u)-V(D v)|^{2 q / p}\right) d x \\
& \stackrel{(6.15)}{\leq} c|h|^{\frac{\beta 2 q}{p}} \int_{\hat{B}}\left(s^{q}+|D u|^{q}+|\bar{f}|\right) d x .
\end{aligned}
$$

We recall that $16 B=\hat{B}$. Summarizing the latter estimate and (6.15) yields

$$
I I+I I I \leq c\left[|h|^{\beta \sigma(q, \theta)}+|h|^{\beta 2 q / p}\right] \int_{\hat{B}}\left(s^{q}+|D u|^{q}+|\bar{f}|\right) d x,
$$

where $c \equiv c(n, p, L / v, q)$ is independent of any of the balls considered. Recalling (6.1) and Remark 6.1 that gives $\delta \leq 1$, we estimate $|h|^{\beta 2 q / p} \leq|h|^{\beta \sigma(q, \theta)}=$ $|h|^{\beta \delta 2 q / p}$ as $|h| \leq 1$, therefore

$$
I I+I I I \leq c|h|^{\beta \delta 2 q / p} \int_{\hat{B}}\left(s^{q}+|D u|^{q}+|\bar{f}|\right) d x .
$$

Implicit in the previous inequality is

$$
\int_{8 B}\left|V(D u)-V\left(D v_{0}\right)\right|^{2 q / p} d x \leq c|h|^{\beta \delta 2 q / p} \int_{\hat{B}}\left(s^{q}+|D u|^{q}+|\bar{f}|\right) d x .
$$

Now we turn to $I$. Applying Lemma 3.2 to $v_{0}$ taking $a_{0}(z) \equiv a\left(x_{0}, z\right),(3.1)$ gives

$$
f_{2 B}\left|D\left(V\left(D v_{0}\right)\right)\right|^{2} d x \leq c|h|^{-2 \beta} f_{4 B}\left|V\left(D v_{0}\right)-V\left(z_{0}\right)\right|^{2} d x,
$$


for every $z_{0} \in \mathbb{R}^{n}$, while using (3.2) with $t=q / p$, we also have

$$
\left(f_{4 B}\left|V\left(D v_{0}\right)-V\left(z_{0}\right)\right|^{2} d x\right)^{\frac{q}{p}} \leq c f_{8 B}\left|V\left(D v_{0}\right)-V\left(z_{0}\right)\right|^{2 q / p} d x .
$$

Now, again using Hölder's inequality yields

$$
\int_{B}\left|\tau_{i, h} V\left(D v_{0}\right)\right|^{2 q / p} d x \leq c|h|^{n \beta(1-q / p)}\left(\int_{B}\left|\tau_{i, h} V\left(D v_{0}\right)\right|^{2} d x\right)^{\frac{q}{p}} .
$$

Using the definition of the operator $\tau_{i, h}$ in (2.10), elementary properties of Sobolev functions, and again the restriction on $|h|$ imposed in (6.10) that in this case serves to ensure that $B\left(x_{0},|h|^{\beta}\right)+B(0,|h|) \subset B\left(x_{0}, 2|h|^{\beta}\right)$, we have

$$
\begin{aligned}
\int_{B}\left|\tau_{i, h} V\left(D v_{0}\right)\right|^{2} d x & \leq c|h|^{2} \int_{2 B}\left|D V\left(D v_{0}\right)\right|^{2} d x \\
& \stackrel{(6.19)}{\leq} c|h|^{2-2 \beta} \int_{4 B}\left|V\left(D v_{0}\right)-V\left(z_{0}\right)\right|^{2} d x .
\end{aligned}
$$

Combining (6.21) and (6.22) gives

$$
\int_{B}\left|\tau_{i, h} V\left(D v_{0}\right)\right|^{2 q / p} d x \leq c|h|^{(1-\beta) 2 q / p+n \beta(1-q / p)}\left(\int_{4 B}\left|V\left(D v_{0}\right)-V\left(z_{0}\right)\right|^{2} d x\right)^{\frac{q}{p}} .
$$

Using now (6.20) gives with $c \equiv c(n, p, L / \nu, q)$

$$
I=c \int_{B}\left|\tau_{i, h} V\left(D v_{0}\right)\right|^{2 q / p} d x \leq c|h|^{(1-\beta) 2 q / p} \int_{8 B}\left|V\left(D v_{0}\right)-V\left(z_{0}\right)\right|^{2 q / p} d x,
$$

and we estimate the last integral; recall that in the latter estimate $z_{0} \in \mathbb{R}^{n}$ is still to be chosen. We shall distinguish two cases now.

Case $t=0$. In this case we take $z_{0}=0$ in (6.23); then (6.18) and (2.3) yield

$$
\begin{aligned}
\int_{8 B} \mid V & \left(D v_{0}\right)-\left.V\left(z_{0}\right)\right|^{2 q / p} d x \leq c \int_{8 B}\left(s^{q}+\left|D v_{0}\right|^{q}\right) d x \\
& \leq c \int_{8 B}\left(s^{q}+|D u|^{q}\right) d x+c \int_{8 B}\left|V(D u)-V\left(D v_{0}\right)\right|^{2 q / p} d x \\
& \leq c \int_{\hat{B}}\left(s^{q}+|D u|^{q}+|\bar{f}|\right) d x .
\end{aligned}
$$

Case $t>0$. In this case we choose $z_{0}$ as the following "average":

$$
z_{0}:=V^{-1}\left((V(D u))_{8 B}\right) ;
$$


observe that such a choice is possible since the map $V$ is bijective. Now, first

$$
\begin{aligned}
\int_{8 B}\left|V\left(D v_{0}\right)-V\left(z_{0}\right)\right|^{2 q / p} d x \leq & c \int_{8 B}\left|V\left(D v_{0}\right)-V(D u)\right|^{2 q / p} d x \\
& +c \int_{8 B}\left|V(D u)-V\left(z_{0}\right)\right|^{2 q / p} d x .
\end{aligned}
$$

Then by (6.4) and Proposition 2.3 with (6.25), we have

$$
\int_{8 B}\left|V(D u)-V\left(z_{0}\right)\right|^{2 q / p} d x \leq c|h|^{\beta t 2 q / p}[V(D u)]_{t, 2 q / p ; 8 B}^{2 q / p} .
$$

Combining (6.27) and (6.18) with (6.26) we have

$$
\begin{aligned}
\int_{8 B}\left|V\left(D v_{0}\right)-V\left(z_{0}\right)\right|^{2 q / p} d x \\
\quad \leq c|h|^{\beta t 2 q / p}\left\{\int_{\hat{B}}\left(s^{q}+|D u|^{q}+|\bar{f}|\right) d x+[V(D u)]_{t, 2 q / p ; \hat{B}}^{2 q / p}\right\} .
\end{aligned}
$$

Observe that we have used $t<\delta$ to estimate $|h|^{\beta \delta 2 q / p} \leq|h|^{\beta t 2 q / p}$ as $|h| \leq 1$.

Now let us define for any measurable set $A \subset \subset \Omega$ the following set function:

$$
\lambda(A):=\int_{A}\left(s^{q}+|D u|^{q}+|\bar{f}|\right) d x+\chi(t)[V(D u)]_{t, 2 q / p ; A}^{2 q / p},
$$

where $\chi(t)=0$ if $t=0$, and $\chi(t)=1$ if $t>0$. Summarizing (6.23), (6.24) and (6.28) we have

$$
I=c \int_{B}\left|\tau_{i, h} V\left(D v_{0}\right)\right|^{2 q / p} d x \leq c|h|^{[(1-\beta)+t \beta] 2 q / p} \lambda(\hat{B}) .
$$

Combining the latter estimate with (6.17), and in turn with (6.14), we find

$$
\int_{B}\left|\tau_{i, h} V(D u)\right|^{2 q / p} d x \leq c\left[|h|^{[(1-\beta)+t \beta] 2 q / p}+|h|^{\beta \delta 2 q / p}\right] \lambda(\hat{B}) .
$$

Since by (6.9) $Q_{\text {inn }}(B) \equiv Q_{\text {inn }} \subset B$ and $\hat{B} \subset \hat{Q}_{\text {out }} \equiv Q_{\text {out }}(\hat{B})$, we finally obtain

$$
\int_{Q_{\mathrm{inn}}}\left|\tau_{i, h} V(D u)\right|^{2 q / p} d x \leq \tilde{c}\left[|h|^{[(1-\beta)+t \beta] 2 q / p}+|h|^{\beta \delta 2 q / p}\right] \lambda\left(\hat{Q}_{\text {out }}\right) .
$$

Now we conclude with a covering argument. Preliminary, observe that the set function $\lambda(\cdot)$ in (6.29) is not a measure due to the presence of $[V(D u)]_{t, 2 q / p ; A}$ in its definition, but it is nevertheless countably super-additive, that is

$$
\sum \lambda\left(A_{j}\right) \leq \lambda\left(\cup A_{j}\right)
$$


whenever $\left\{A_{j}\right\}_{j}$ is a countable family of mutually disjoint subsets. The covering argument goes now as follows: first recall that all the cubes here have sides parallel to the coordinate axes; then for each $h \in \mathbb{R} \backslash\{0\}$ satisfying (6.10) we can find balls $B_{1} \equiv B\left(x_{1},|h|^{\beta}\right), \ldots, B_{J} \equiv B\left(x_{J},|h|^{\beta}\right), J \equiv J(h) \in \mathbb{N}$ of the type considered in (6.11) such that the corresponding inner cubes $Q_{\text {inn }}\left(B_{1}\right), \ldots, Q_{\text {inn }}\left(B_{J}\right)$ are disjoint and cover $\Omega^{\prime}$ up to a negligible set

$$
\left|\Omega^{\prime} \backslash \bigcup Q_{\text {inn }}\left(B_{j}\right)\right|=0, \quad Q_{\text {inn }}\left(B_{i}\right) \cap Q_{\text {inn }}\left(B_{j}\right) \neq \emptyset \Longleftrightarrow i=j .
$$

Actually we are proceeding as follows: we first take a lattice of cubes $\left\{Q_{j}\right\}$ with equal side length, comparable to $|h|^{\beta}$, and sides parallel to the coordinate axes, in order to obtain (6.32). They must be centered in $\Omega^{\prime}$. Then we view them as the inner cubes of the balls $\left\{B\left(x_{j},|h|^{\beta}\right)\right\}$, according to (6.9). Now we sum up inequalities (6.30) for $j \leq J$ and get

$$
\begin{aligned}
& \sum \int_{Q_{\text {inn }}\left(B_{j}\right)}\left|\tau_{i, h} V(D u)\right|^{2 q / p} d x \\
& \leq \tilde{c}\left[|h|^{[(1-\beta)+t \beta] 2 q / p}+|h|^{\beta \delta 2 q / p}\right] \sum \lambda\left(Q_{\text {out }}\left(\hat{B}_{j}\right)\right) .
\end{aligned}
$$

By construction, and in particular by (6.10), we have $Q_{\text {out }}\left(\hat{B}_{j}\right) \subset \Omega^{\prime \prime}$, for every $j \leq J$. Moreover by (6.32) each of the dilated outer cubes $Q_{\text {out }}\left(\hat{B}_{j}\right)$ intersects the similar ones $Q_{\text {out }}\left(\hat{B}_{k}\right)$ less than $(32 \sqrt{n})^{n}$ times. Using all these facts and (6.31), in turns out that (6.32)-(6.33) imply

$$
\int_{\Omega^{\prime}}\left|\tau_{i, h} V(D u)\right|^{2 q / p} d x \leq 2^{8 n} \tilde{c}\left[|h|^{[(1-\beta)+t \beta] 2 q / p}+|h|^{\beta \delta 2 q / p}\right] \lambda\left(\Omega^{\prime \prime}\right) .
$$

Now we determine $\beta$ in order to minimize the right-hand side with respect to $|h|$; this yields $[(1-\beta)+t \beta]=\beta \delta$, that is $\beta=\gamma(t) / \delta$, see (6.1). Observe that we are requiring everywhere that $\beta<1$, see (6.10), and the choice $\beta=\gamma(t) / \delta$ is admissible since $t<\delta$ implies $\gamma(t) / \delta<1$. Accordingly, for any $h$ as in (6.10), (6.34) becomes

$$
\int_{\Omega^{\prime}}\left|\tau_{i, h} V(D u)\right|^{2 q / p} d x \leq c_{0}|h|^{\gamma(t) 2 q / p} \lambda\left(\Omega^{\prime \prime}\right),
$$

for $c_{0} \equiv c_{0}(n, p, L / \nu, q)$. Therefore, since $i \in\{1, \ldots, n\}$ is arbitrary, the crucial inequality (2.11) of Lemma 2.1 is satisfied with $d$ as in (6.10), $q$ replaced by $2 q / p$, $\bar{\alpha} \equiv \gamma(t)$, and finally $S \equiv\left[c_{0} \lambda\left(\Omega^{\prime \prime}\right)\right]^{p / 2 q}$. Up to changing the subsets according to Lemma 2.13, that is passing to inner and outer subsets to $\Omega^{\prime \prime}$ and $\Omega^{\prime}$ respectively, we may apply Lemma 2.1 that now gives $V(D u) \in W_{\text {loc }}^{\tilde{t}, 2 q / p}\left(\Omega^{\prime}, \mathbb{R}^{n}\right)$, for every $\tilde{t}<\gamma(t)$; as $\Omega^{\prime}$ is arbitrary, this proves the first part of the assertion. Changing again the subsets, since $\Omega^{\prime} \subset \subset \Omega^{\prime \prime}$ are themselves arbitrary, using estimate (2.12), 
and finally (2.3), we have that for every couple of open subsets $\Omega^{\prime} \subset \subset \Omega^{\prime \prime}$ there exists a constant $c \equiv c\left(n, p, L / \nu, q, \operatorname{dist}\left(\Omega^{\prime}, \partial \Omega^{\prime \prime}\right)\right)$ such that

$$
[V(D u)]_{\tilde{t}, 2 q / p ; \Omega^{\prime}}^{2 q / p} \leq c \int_{\Omega^{\prime \prime}}\left(s^{q}+|D u|^{q}+|\bar{f}|\right) d x+c[V(D u)]_{t, 2 q / p ; \Omega^{\prime \prime}}^{2 q / p} .
$$

We have used (2.3) to estimate the integral of $V$ arising when applying (2.12):

$$
\int_{\Omega^{\prime \prime}}|V(D u)|^{2 q / p} d x \leq c \int_{\Omega^{\prime \prime}}\left(s^{q}+|D u|^{q}\right) d x .
$$

Using (6.36) in combination with (6.5), and again changing the subsets via Lemma 2.13, we finally obtain (6.7) with the specified dependence of $c$. In a completely similar way using (6.35) it follows (6.8) with $|h| \leq d$ as in (6.10). The full case $0<|h|<\operatorname{dist}\left(\Omega^{\prime}, \partial \Omega^{\prime \prime}\right)$ follows by increasing the constant $c$ in (6.35) by a number depending on $n, p, q$ and $\operatorname{dist}\left(\Omega^{\prime}, \partial \Omega^{\prime \prime}\right)$; indeed when $|h| \in\left(d, \operatorname{dist}\left(\Omega^{\prime}, \partial \Omega^{\prime \prime}\right)\right)$

$$
\begin{aligned}
\sup _{h} & \int_{\Omega^{\prime}} \frac{\left|\tau_{i, h} V(D u(x))\right|^{2 q / p}}{|h|^{\gamma(t) 2 q / p}} d x \\
& \leq \frac{c}{d \gamma(t) 2 q / p} \int_{\Omega^{\prime}}\left|V\left(D u\left(x+h e_{i}\right)\right)\right|^{2 q / p}+|V(D u(x))|^{2 q / p} d x \\
& \leq \frac{c}{d^{\gamma(t) 2 q / p}} \int_{\Omega^{\prime \prime}}|V(D u)|^{2 q / p} d x \stackrel{(2.3)}{\leq} \frac{c}{d^{\gamma(t) 2 q / p}} \int_{\Omega^{\prime \prime}}\left(s^{q}+|D u|^{q}\right) d x
\end{aligned}
$$

The proof, also of (6.6), is complete as the open subsets considered are arbitrary.

Lemma 6.3. Let $u \in W_{0}^{1, p}\left(\Omega, \mathbb{R}^{N}\right)$ be the unique solution to (4.2), under the assumptions (1.2) and (1.4) with $p \leq n$, and let $q$ be such that $p-1 \leq q<b$. Then

$$
V(D u) \in W_{\mathrm{loc}}^{t, 2 q / p}\left(\Omega, \mathbb{R}^{n}\right), \quad D u \in W_{\mathrm{loc}}^{2 t / p, q}\left(\Omega, \mathbb{R}^{n}\right), \text { for every } t \in[0, \delta),
$$

where $\delta$ is in (6.1). Moreover, for every couple of open subsets $\Omega^{\prime} \subset \subset \Omega^{\prime \prime} \subset \subset \Omega$ there exists a constant $c \equiv c\left(n, p, L / \nu, q, t, \operatorname{dist}\left(\Omega^{\prime}, \partial \Omega^{\prime \prime}\right)\right)$ such that

$$
[V(D u)]_{t, 2 q / p ; \Omega^{\prime}}^{2 q / p}+[D u]_{2 t / p, q ; \Omega^{\prime}}^{q} \leq c \int_{\Omega^{\prime \prime}}\left(|D u|^{q}+s^{q}+|\bar{f}|\right) d x
$$

and

$$
\sup _{h} \int_{\Omega^{\prime}} \frac{\left|\tau_{i, h} D u(x)\right|^{q}}{|h|^{t 2 q / p}} d x \leq c \int_{\Omega^{\prime \prime}}\left(|D u|^{q}+s^{q}+|\bar{f}|\right) d x,
$$

for every $i \in\{1, \ldots, n\}$, where $0<|h|<\operatorname{dist}\left(\Omega^{\prime}, \partial \Omega^{\prime \prime}\right)$. 
Proof. The proof follows from Lemma 6.2 via iteration. We first prove the assertion about $V(D u)$. The function $\gamma(\cdot)$ in (6.1) is seen to be increasing and it satisfies

$$
t \in(0, \delta) \Longrightarrow \gamma(t) \in(t, \delta) \quad \text { and } \quad \gamma(\delta)=\delta
$$

Now, let us inductively define the two sequences $\left\{t_{k}\right\}_{k \geq 1}$ and $\left\{s_{k}\right\}_{k \geq 1}$ as

$$
s_{1}:=\frac{\delta}{4(\delta+1)}, \quad t_{1}=2 s_{1}, \quad s_{k+1}:=\gamma\left(s_{k}\right), \quad t_{k+1}:=\frac{\gamma\left(s_{k}\right)+\gamma\left(t_{k}\right)}{2} .
$$

From (6.41) it follows that $s_{k} \nearrow \delta$, moreover, since $\gamma(\cdot)$ is increasing we have that $s_{k}<t_{k}<\delta$, so that also $t_{k} \nearrow \delta$ holds. We prove by induction that $V(D u) \in$ $W_{\text {loc }}^{t_{k}, 2 q / p}\left(\Omega, \mathbb{R}^{n}\right)$, for every $k \in \mathbb{N}$; this will prove the first assertion in (6.38). Applying Lemma 6.2 with $t=0$ we immediately get $V(D u) \in W_{\text {loc }}^{t_{1}, 2 q / p}\left(\Omega, \mathbb{R}^{n}\right)$, with a corresponding estimate of the type (6.5). Now assuming that $V(D u) \in$ $W_{\text {loc }}^{t_{k}, 2 q / p}\left(\Omega, \mathbb{R}^{n}\right)$, we may apply again Lemma 6.2 with $t=t_{k}$, to get that $V(D u) \in$ $W_{\text {loc }}^{t, 2 q / p}\left(\Omega, \mathbb{R}^{n}\right)$ for every $t<\gamma\left(t_{k}\right)$. Now observe, that since $\gamma(\cdot)$ is increasing and $s_{k}<t_{k}$, we have that $t_{k+1}<\gamma\left(t_{k}\right)$, and therefore $V(D u) \in W_{\mathrm{loc}}^{t_{k+1}, 2 q / p}\left(\Omega, \mathbb{R}^{n}\right)$, with corresponding estimates of the type (6.7) and (6.8). Taking into account the fact that the open subsets $\Omega^{\prime} \subset \subset \Omega^{\prime \prime} \subset \subset \Omega$ in Lemma 6.2 are arbitrary, and the estimates (6.5) and (6.7), the part of (6.39) regarding $V(D u)$ also follows by induction. In the same way, by induction on (6.8), for every $i \in\{1, \ldots, n\}$ and considering $0<|h|<\operatorname{dist}\left(\Omega^{\prime}, \partial \Omega^{\prime \prime}\right)$, we have

$$
\sup _{h} \int_{\Omega^{\prime}} \frac{\left|\tau_{i, h} V(D u(x))\right|^{2 q / p}}{|h|^{t 2 q / p}} d x \leq c \int_{\Omega^{\prime \prime}}\left(|D u|^{q}+s^{q}+|\bar{f}|\right) d x, \quad \forall t<\delta .
$$

The assertions concerning $D u$ instead follows using (2.2) and the fact that $p \geq 2$ :

$$
\begin{aligned}
{[D u]_{\frac{2 t}{p}, q ; \Omega^{\prime}}^{q} } & =\int_{\Omega^{\prime}} \int_{\Omega^{\prime}} \frac{|D u(x)-D u(y)|^{q}}{|x-y|^{n+2 t q / p}} d x d y \\
& \leq \int_{\Omega^{\prime}} \int_{\Omega^{\prime}} \frac{\left[(s+|D u(x)|+|D u(y)|)^{p-2}|D u(x)-D u(y)|^{2}\right]^{q / p}}{|x-y|^{n+2 t q / p}} d x d y \\
& \leq c \int_{\Omega^{\prime}} \int_{\Omega^{\prime}} \frac{|V(D u(x))-V(D u(y))|^{2 q / p}}{|x-y|^{n+2 t q / p}} d x d y \\
& =c[V(D u)]_{t, 2 q / p ; \Omega^{\prime}}^{2 q / p}
\end{aligned}
$$

for any $\Omega^{\prime} \subset \subset \Omega$, where $c \equiv c(n, p)$; this gives (6.39). A completely similar argument allows to get (6.40) from (6.43), and the proof is complete.

Proof of Theorems 1.2 and 1.3. Firstly, observe that since $p \geq 2$, then $q \geq p-1$ implies $2 q / p \geq 1$, and therefore Lemma 6.3 can be used in the full range (1.15). 
We consider the approximation sequence $\left\{u_{k}\right\}_{k}$ built in Section 5. Applying to each $u_{k}$ the result of Lemma 6.3, and keeping in mind (5.3)-(5.5), we have

$$
\left\|D u_{k}\right\|_{W^{\sigma / q, q}\left(\Omega^{\prime}\right)}^{q} \leq c \int_{\Omega}\left(s^{q}+\left|D u_{k}\right|^{q}+\left|\bar{f}_{k}\right|\right) d x \leq c[|\mu|(\Omega)]^{\frac{q}{p-1}}+c s^{q}|\Omega|,
$$

with the obvious definition of $\bar{f}_{k}:=\left\|f_{k}\right\|_{L^{1}(\Omega)}^{\frac{q-p+1}{p-1}} f_{k}$ : look at (6.2)-(6.3) and recall that here it is $\theta=n$. The constant $c$ depends as in the statement of Theorem 1.2, while $q \in[p-1, b)$, and $\sigma \in(0, \sigma(q))$. Now estimate (1.17) follows from (5.5),(5.6) and (6.45), together with a standard lower semicontinuity argument to handle the left-hand sides of (5.5), (6.45). We conclude showing that $u$ solves (1.1) in the sense of (1.7). The a priori estimate (6.45) allows for a quick derivation of this fact. Indeed, thanks to Rellich's compactness theorem in the case of fractional Sobolev spaces [4], we have that, up to extracting a diagonal subsequence, $D u_{k}$ strongly converges to $D u$ in $L_{\text {loc }}^{t}\left(\Omega, \mathbb{R}^{n}\right)$ for every $t<n q /(n-\sigma(q))$, and on the other hand note that $n q /(n-\sigma(q))=n(p-1) /(n-1)>p-1$. Taking into account the growth condition (2.8), and that $f_{k} \rightarrow \mu$ by (5.2), we can pass to the limit in $(5.1)_{1}$ using (2.8) and a well known variant of Lebesgue's dominated convergence theorem, getting that $u$ finally satisfies (1.7). The proof of Theorem 1.2 is now complete, and estimate (1.17) is also proved. It remains to prove (1.16), to this aim we use a scaling argument. Take $B_{R} \subset \Omega$, let $u \in W^{1, p}(\Omega)$ be the solution to (4.2) with a fixed $f$, and scale it back as in (4.5) in order to obtain $\tilde{u}(y)$, a solution in $B_{1}$. Now observe that we may apply Lemma 6.3 to $\tilde{u}$ since the whole argument of the lemma is local, and makes no use of the boundary information on the solution considered. Therefore estimate (6.39) applied to $\tilde{u}$ with $\Omega^{\prime} \equiv B_{1 / 2}$ gives

$$
[D \tilde{u}]_{\sigma / q, q ; B_{1 / 2}}^{q} \leq c\||D \tilde{u}|+s\|_{L^{q}\left(B_{1}\right)}^{q}+c\|\tilde{f}\|_{L^{1}\left(B_{1}\right)}^{q /(p-1)}
$$

for every $\sigma<\sigma(q)$; here we also used (6.3) while $c \equiv c(n, p, L / v, \sigma, q)$. Scaling back to $B_{R}$, observing that $[D \tilde{u}]_{\sigma / q, q ; B_{1 / 2}}^{q}=R^{\sigma-n}[D u]_{\sigma / q, q ; B_{R / 2}}^{q}$ we have

$$
[D u]_{\sigma / q, q ; B_{R / 2}}^{q} \leq c R^{-\sigma}\||D u|+s\|_{L^{q}\left(B_{R}\right)}^{q}+c R^{\sigma(q)-\sigma}\|f\|_{L^{1}\left(B_{R}\right)}^{q /(p-1)} .
$$

We used that $n-\sigma(q)=q(n-1) /(p-1)$ by (4.1). Writing the latter estimate for $u \equiv u_{k}$, and using the approximation scheme of Section 5 and in particular (5.2) and (5.4), we finally obtain estimate (1.16).

Remark 6.4. The crucial case in the proof Theorem 1.2 is actually (1.12). The case (1.14) can be obtained by embedding from (1.12) [65], 2.2.3. Indeed, for a space $W^{\alpha, q}$ the number $\alpha-n / q$ is called integer dimension; all the spaces in (1.14) share the same integer dimension if $\varepsilon=0$, and this allows for using a suitable embedding. We gave here a self-contained proof, which is on the other hand even shorter than the one using abstract embedding theorems for Besov spaces. 
Proof of Theorem 1.4. The proof goes along the lines of the one for Theorem 1.2. Take $q=p-1$ in Lemma 6.3, in such a way that now (6.1) gives

$$
\delta=\frac{p}{2(p-1)} .
$$

Now we proceed as for the proof of Theorems 1.2-1.3, again applying Lemma 6.3 first to the approximating solutions $u_{k}$ defined in Section 5, and then passing to the limit $k \nearrow \infty$ the resulting a priori estimates. The equality in (6.46) together with (6.38) finally leads to

$$
V(D u) \in W_{\mathrm{loc}}^{t, \frac{2(p-1)}{p}}\left(\Omega, \mathbb{R}^{n}\right) \quad \text { for every } \quad t<\frac{p}{2(p-1)},
$$

which establishes (1.22) in Theorem 1.4. In order to get (1.23) and therefore completing the proof we just use the a priori estimate (6.39) for the approximate solutions $u_{k}$, and then we let $k \nearrow \infty$ as for the proof of Theorem 1.3.

Remark 6.5. As for (1.15) we can prove, using Lemma 6.3, that the solution $u$ found in Theorem 1.4 satisfies

$$
V(D u) \in W_{\text {loc }}^{\frac{p \sigma(q)}{2 q}-\varepsilon, \frac{2 q}{p}}\left(\Omega, \mathbb{R}^{n}\right), \quad \text { for every } \varepsilon>0,
$$

for the values of $q$ described in (1.15).

Proof of Corollary 1.5. This is based on inequality (2.2). Set $q_{0}=2(p-1) / p$; since $p \geq 2$ we have

$$
\begin{aligned}
& s^{\frac{(p-2) q_{0}}{2}} \int_{\Omega^{\prime}} \int_{\Omega^{\prime}} \frac{|D u(x)-D u(y)|^{q_{0}}}{|x-y|^{n+1-\varepsilon}} d x d y \\
& \quad \leq \int_{\Omega^{\prime}} \int_{\Omega^{\prime}} \frac{\left[(s+|D u(x)|+|D u(y)|)^{p-2}|D u(x)-D u(y)|^{2}\right]^{q_{0} / 2}}{|x-y|^{n+1-\varepsilon}} d x d y \\
& \quad \leq c(n, p) \int_{\Omega^{\prime}} \int_{\Omega^{\prime}} \frac{|V(D u(x))-V(D u(y))|^{q_{0}}}{|x-y|^{n+1-\varepsilon}} d x d y,
\end{aligned}
$$

and the proof is concluded using estimate (1.23).

\section{The capacitary case}

Here we give the proof of Theorem 1.10, that will be along the lines of the one for Theorem 1.2; therefore we shall confine to report the necessary modifications. The main point here is that we do not need estimates below the growth exponent like (3.2) and (3.11), as the solution $u$ to (1.1) is uniquely determined in $W_{0}^{1, p}(\Omega)$; for the same reason no approximation scheme as in Section 5 is needed. 
As for (6.1) we first we need to define

$$
\delta:=\frac{\sigma(p)}{2}, \quad \gamma(t):=\frac{\delta}{\delta+1-t} \quad t \in[0, \delta+1), \quad \bar{\mu}:=M^{\frac{1}{p-1}}|\mu|,
$$

where $M$ appears in (1.27), and $\sigma(p)$ is defined in (1.36). Next lemma is the analog of Lemma 6.2.

Lemma 7.1. Let $u \in W_{0}^{1, p}\left(\Omega, \mathbb{R}^{N}\right)$ be the unique solution to (1.1), under the assumptions (1.2), (1.4) and (1.27) for $\theta<p$. Assume that

$$
V(D u) \in W_{\mathrm{loc}}^{t, 2}\left(\Omega, \mathbb{R}^{n}\right), \quad \text { for some } t \in[0, \delta),
$$

where $\delta$ is as in (7.1), and that for every couple of open subsets $\Omega^{\prime} \subset \subset \Omega^{\prime \prime} \subset \subset \Omega$ there exists a constant $c_{1} \equiv c_{1}\left(\operatorname{dist}\left(\Omega^{\prime}, \partial \Omega^{\prime \prime}\right)\right)$ such that

$$
[V(D u)]_{t, 2 ; \Omega^{\prime}}^{2} \leq c_{1} \int_{\Omega^{\prime \prime}}\left(|D u|^{p}+s^{p}\right) d x+c_{1} \bar{\mu}\left(\Omega^{\prime \prime}\right)
$$

Then

$$
V(D u) \in W_{\text {loc }}^{\tilde{t}, 2}\left(\Omega, \mathbb{R}^{n}\right), \quad \text { for every } \tilde{t} \in[0, \gamma(t)),
$$

where $\gamma(\cdot)$ is in (7.1). Moreover, for every couple of open subsets $\Omega^{\prime} \subset \subset \Omega^{\prime \prime} \subset \subset \Omega$ there exists a constant $c \equiv c\left(n, p, L / v, \operatorname{dist}\left(\Omega^{\prime}, \partial \Omega^{\prime \prime}\right), \tilde{t}, c_{1}\right)$ such that

$$
[V(D u)]_{\tilde{t}, 2 ; \Omega^{\prime}}^{2} \leq c \int_{\Omega^{\prime \prime}}\left(|D u|^{p}+s^{p}\right) d x+c \bar{\mu}\left(\Omega^{\prime \prime}\right) .
$$

Proof. The proof follows the one of Lemma 6.2, therefore we shall keep the notation introduced there, giving the suitable modifications. Let us firstly treat the case $p \neq n$. Once again $h, v, v_{0}$ are as in (6.10) and (6.12)-(6.13), respectively. As for (6.14),

$$
\begin{aligned}
\int_{B}\left|\tau_{i, h} V(D u)\right|^{2} d x \leq c & \int_{B}\left|\tau_{i, h} V\left(D v_{0}\right)\right|^{2} d x+c \int_{\hat{B}}|V(D u)-V(D v)|^{2} d x \\
& +c \int_{2 B}\left|V(D v)-V\left(D v_{0}\right)\right|^{2} d x \\
= & : I+I I+I I I .
\end{aligned}
$$

The term III is estimated via (6.16), while for II we use Lemmata 4.4-4.5:

$$
\int_{\hat{B}}|V(D u)-V(D v)|^{2} d x \leq c \bar{\mu}(\hat{B})|h|^{\beta \sigma(p)} .
$$

Therefore, as $\sigma(p) \leq 2$ when $p \geq 2$, we have

$$
\int_{\hat{B}}\left|V(D u)-V\left(D v_{0}\right)\right|^{2} d x+I I+I I I \leq c|h|^{\beta 2 \delta}\left\{\int_{\hat{B}}\left(s^{p}+|D u|^{p}\right) d x+\bar{\mu}(\hat{B})\right\} .
$$


As for $I$ we shall simply use estimate (6.22). We again distinguish two cases:

Case $t=0$. Taking $z_{0}=0$ we have, using (7.7) and (2.3)

$$
\begin{aligned}
\int_{8 B}\left|V\left(D v_{0}\right)-V\left(z_{0}\right)\right|^{2} d x & \leq c \int_{\hat{B}}\left(s^{p}+|D u|^{p}\right) d x+\int_{8 B}\left|V(D u)-V\left(D v_{0}\right)\right|^{2} d x \\
& \leq c \int_{\hat{B}}\left(s^{p}+|D u|^{p}\right) d x+c \bar{\mu}(\hat{B}) .
\end{aligned}
$$

Case $t>0$. In this case we choose $z_{0}$ as in (6.25). Again we estimate

$$
\begin{aligned}
\int_{8 B}\left|V\left(D v_{0}\right)-V\left(z_{0}\right)\right|^{2} d x \leq c \int_{8 B}\left|V\left(D v_{0}\right)-V(D u)\right|^{2} d x \\
+c \int_{8 B}\left|V(D u)-V\left(z_{0}\right)\right|^{2} d x .
\end{aligned}
$$

Using Proposition 2.3, together with (7.2) and the choice (6.25), gives

$$
\int_{8 B}\left|V(D u)-V\left(z_{0}\right)\right|^{2} d x \leq c|h|^{\beta 2 t}[V(D u)]_{t, 2 ; 8 B}^{2} .
$$

Combining (7.10) and (7.7) with (7.9) we have, as $t<\delta$

$$
\begin{aligned}
& \int_{8 B}\left|V\left(D v_{0}\right)-V\left(z_{0}\right)\right|^{2} d x \\
& \quad \leq c|h|^{\beta 2 t}\left[\int_{\hat{B}}\left(s^{p}+|D u|^{p}\right) d x+\bar{\mu}(\hat{B})+[V(D u)]_{t, 2 ; \hat{B}}^{2}\right] .
\end{aligned}
$$

Now let us set for any measurable set $A \subset \subset \Omega$

$$
\lambda(A):=\int_{A}\left(s^{p}+|D u|^{p}\right) d x+\bar{\mu}(A)+\chi(t)[V(D u)]_{t, 2 ; A}^{2},
$$

where again $\chi(t)=0$ if $t=0$, and $\chi(t)=1$ if $t>0$. Summarizing (7.6), (7.8) and (7.11) we have

$$
I \leq c \int_{B}\left|\tau_{i, h} V\left(D v_{0}\right)\right|^{2} d x \leq c|h|^{2[(1-\beta)+t \beta]} \lambda(\hat{B}) .
$$

Combining this last estimate with (7.7) and (7.6) we finally find

$$
\int_{B}\left|\tau_{i, h} V(D u)\right|^{2} d x \leq c\left[|h|^{2[(1-\beta)+t \beta]}+|h|^{\beta 2 \delta}\right] \lambda(\hat{B}) .
$$

From now on we can proceed with the covering argument adopted in the proof of Lemma 6.2, up to formula (6.34), arriving at

$$
\int_{\Omega^{\prime}}\left|\tau_{i, h} V(D u)\right|^{2} d x \leq c\left[|h|^{2[(1-\beta)+t \beta]}+|h|^{\beta 2 \delta}\right] \lambda\left(\Omega^{\prime \prime}\right) .
$$


Taking $\beta=\gamma(t) / \delta \in(0,1)$ now yields

$$
\int_{\Omega^{\prime}}\left|\tau_{i, h} V(D u)\right|^{2} d x \leq c|h|^{2 \gamma(t)} \lambda\left(\Omega^{\prime \prime}\right),
$$

that is the analog of (6.35). From this point on the proof proceeds as for Lemma 6.2, and the case $p \neq n$ is complete. As for $p=n$, Lemma 4.5 allows to re-do the whole proof where this time $\delta:=\sigma^{\prime} / 2$, for any $\sigma^{\prime} \in(0, \sigma(p))$; therefore we obtain $V(D u) \in W_{\mathrm{loc}}^{\tilde{t}, 2}\left(\Omega, \mathbb{R}^{n}\right)$ for every $\tilde{t}<\left(\sigma^{\prime} / 2\right) /\left[\left(\sigma^{\prime} / 2\right)+1-t\right]$. Since $\sigma^{\prime}$ can be chosen arbitrarily close to $\sigma(p)$ the statement follows again, and the proof is complete. In particular (7.4) follows from the fact that the open subsets considered are arbitrary.

Proof of Theorem 1.10. The proof goes as the one for Lemma 6.3, but directly for the solution $u$ to (1.1). Applying repeatedly Lemma 7.1 with $t \equiv t_{k}$ as in Lemma 6.3, and $\left\{t_{k}\right\}$ is the sequence defined in (6.42) with $\delta=\sigma(p) / 2$, we get that $V(D u) \in W_{\text {loc }}^{t_{k}, 2}\left(\Omega, \mathbb{R}^{n}\right)$ for every $k \in \mathbb{N}$, with a corresponding estimate of the type (7.3). The assertion finally follows observing that this time $t_{k} \nearrow \sigma(p) / 2$, passing to from $V(D u)$ to $D u$ as in (6.44), and using (4.26) to get the global bound in (1.37).

\section{Morrey estimates}

In this section we give the proofs of Theorems 1.11 and 1.12. We shall actually argue as follows: we first prove Theorem 1.11 in the special case $q<b$, at least as a priori estimate. This will allows us to prove Theorem 1.12 immediately, and also Theorem 1.8 in the next section. In turn Theorem 1.8 will finally imply Theorem 1.11 for the full range $q<m$; compare with (1.43). Therefore we shall start with

Lemma 8.1. Let $u \in W_{0}^{1, p}(\Omega)$ be the solution to (4.2) for a fixed $f \in L^{\infty}(\Omega)$, under the assumptions (1.2) with $p \leq n$. Then with

$$
p-1 \leq q<\frac{n(p-1)}{n-1}=b, \quad \text { and } \quad \delta(q):=\frac{q(\theta-1)}{p-1}
$$

as in (1.39), for every couple of open subsets $\Omega^{\prime} \subset \subset \Omega^{\prime \prime} \subset \subset \Omega$ there exists $c \equiv$ $c\left(n, p, L / \nu, q, \operatorname{dist}\left(\Omega^{\prime}, \partial \Omega^{\prime \prime}\right)\right)$ such that whenever $\theta \in[p, n]$

$$
\||D u|+s\|_{L^{q, \delta(q)}\left(\Omega^{\prime}\right)} \leq c\||D u|+s\|_{L^{q}\left(\Omega^{\prime \prime}\right)}+c\|f\|_{L^{1, \theta}\left(\Omega^{\prime \prime}\right)}^{1 /(p-1)} .
$$

Moreover there exists $c \equiv c\left(n, p, L / v, q, \operatorname{dist}\left(\Omega^{\prime}, \partial \Omega\right), \Omega\right)$ such that

$$
\||D u|+s\|_{L^{q, \delta(q)}\left(\Omega^{\prime}\right)} \leq c\|f\|_{L^{1}(\Omega)}^{1 /(p-1)}+c\|f\|_{L^{1, \theta}(\Omega)}^{1 /(p-1)}+c s|\Omega|^{1 / q} .
$$


Proof. We shall apply a standard comparison technique to get Morrey estimates. Let us take $B_{R} \subset \subset \Omega^{\prime \prime}$ with $R \leq 1$, and define $v \in u+W_{0}^{1, p}\left(B_{R}\right)$ as the unique solution to (4.3). Using Lemma 3.3, estimate (3.10), for any $\varrho \in(0, R)$

$$
\int_{B_{\varrho}}\left(|D v|^{q}+s^{q}\right) d x \leq c\left(\frac{\varrho}{R}\right)^{n-q+\beta q} \int_{B_{R}}\left(|D v|^{q}+s^{q}\right) d x
$$

where $c \equiv c(n, p, L / \nu, q)$, and $\beta \equiv \beta(n, p, L / \nu) \in(0,1]$. Now we compare $u$ and $v$ in $B_{R}$, that is, using the latter estimate

$$
\begin{gathered}
\int_{B_{\varrho}}\left(|D u|^{q}+s^{q}\right) d x \leq c \int_{B_{\varrho}}\left(|D v|^{q}+s^{q}\right) d x+c \int_{B_{\varrho}}|D v-D u|^{q} d x \\
\leq c\left(\frac{\varrho}{R}\right)^{n-q+\beta q} \int_{B_{R}}\left(|D v|^{q}+s^{q}\right) d x+c \int_{B_{R}}|D v-D u|^{q} d x \\
\leq c\left(\frac{\varrho}{R}\right)^{n-q+\beta q} \int_{B_{R}}\left(|D u|^{q}+s^{q}\right) d x+c \int_{B_{R}}|D v-D u|^{q} d x .
\end{gathered}
$$

Using Lemma 4.3, with $c \equiv c(n, p, L / v, q)$, and $q \in[p-1, b)$, we get

$$
\begin{aligned}
\int_{B_{R}}|D u-D v|^{q} d x & \leq c\|f\|_{L^{1, \theta}\left(B_{R}\right)}^{\frac{q-p+1}{p-1}} \int_{B_{R}}|f| d x R^{\sigma(q, \theta)} \\
& \leq c\|f\|_{L^{1, \theta}\left(B_{R}\right)}^{\frac{q}{p-1}} R^{n-\delta(q)} .
\end{aligned}
$$

Observe that $f \in L^{\infty}$, therefore $\|f\|_{L^{1, \theta}(\Omega)}<\infty$. Combining (8.4) and (8.5) yields

$$
\begin{aligned}
\int_{B_{\varrho}}\left(|D u|^{q}+s^{q}\right) d x \leq & c\left(\frac{\varrho}{R}\right)^{n-q+\beta q} \int_{B_{R}}\left(|D u|^{q}+s^{q}\right) d x \\
& +c\|f\|_{L^{1, \theta}\left(B_{R}\right)}^{\frac{q}{p-1}} R^{n-\delta(q)},
\end{aligned}
$$

where $c \equiv c(n, p, L / v)$. Observe now that $\theta \geq p$ implies $n-q \geq n-\delta(q)$, therefore we can apply Lemma 2.11 with the choice

$$
\varphi(t):=\int_{B_{t}}\left(|D u|^{q}+s^{q}\right) d x, \quad \mathcal{B}:=\|f\|_{L^{1, \theta}\left(B_{R}\right)}^{\frac{q}{p-1}},
$$

and $\delta_{0}:=n-q+\beta q>\delta_{1}:=n-q+\beta q / 2>\gamma \equiv n-\delta(q)$ in order to have, after an elementary manipulation

$$
\begin{aligned}
& \int_{B_{\varrho}}\left(|D u|^{q}+s^{q}\right) d x \\
& \quad \leq c_{1}\left\{c_{*}(R)\left(\frac{\varrho}{R}\right)^{\beta q / 2} \int_{B_{R}}\left(|D u|^{q}+s^{q}\right) d x+\|f\|_{L^{1, \theta}\left(B_{R}\right)}^{\frac{q}{p-1}}\right\} \varrho^{n-\delta(q)},
\end{aligned}
$$


for every $\varrho \leq R$, where $c_{1} \equiv c_{1}(n, p, L / v, q)$, and $c_{*}(R)=R^{\delta(q)-n}$. Now take $\bar{R}:=\operatorname{dist}\left(\Omega^{\prime}, \partial \Omega^{\prime \prime}\right) / 4$, then use (8.7) on the generic ball of radius $\bar{R}$ centered in $\Omega^{\prime}$; of course such a ball is contained in $\Omega^{\prime \prime}$. Also observe that such a choice of $\bar{R}$ determines $c_{*} \equiv c_{*}\left(n, \operatorname{dist}\left(\Omega^{\prime}, \partial \Omega^{\prime \prime}\right)\right)$ in (8.7). All in all such choices give

$$
\int_{B_{\varrho}}\left(|D u|^{q}+s^{q}\right) d x \leq c\left[\||D u|+s\|_{L^{q}\left(\Omega^{\prime \prime}\right)}^{q}+\|f\|_{L^{1, \theta}\left(\Omega^{\prime \prime}\right)}^{\frac{q}{p-1}}\right] \varrho^{n-\delta(q)},
$$

with $c \equiv c\left(n, p, L / \nu, q, \operatorname{dist}\left(\Omega^{\prime}, \partial \Omega^{\prime \prime}\right)\right)$, for any $\varrho \leq \bar{R}$. This procedure, and an elementary estimation involving the definition in (2.14), yield (8.1) with the specified dependence of the constant. More precisely, (8.8) is satisfied for $\varrho \leq \bar{R}$, but then is satisfied also for any ball $B_{\varrho} \subset \Omega^{\prime}$, with $\varrho \leq 1$, modulo increasing the constant $c$ of the factor $\bar{R}^{\delta(q)-n}$ in the case $\bar{R}<1$; recall that $\bar{R}:=\operatorname{dist}\left(\Omega^{\prime}, \partial \Omega^{\prime \prime}\right) / 4$. Finally, in order to get (8.2), fix $\Omega^{\prime} \subset \subset \Omega$, and determine $\Omega^{\prime \prime}$ according to Lemma 2.13; at this point (8.2) follows using (4.25) in (8.1), since $\operatorname{dist}\left(\Omega^{\prime}, \partial \Omega^{\prime \prime}\right)=\operatorname{dist}\left(\Omega^{\prime}, \partial \Omega\right) / 2$.

Proof of Theorem 1.12. Take $\Omega^{\prime} \subset \subset \Omega$ as in the statement of the Theorem, and determine $\Omega^{\prime \prime}$ according to Lemma 2.13. We go back to the proof of Lemma 8.1, and apply the arguments to $u_{k}$, that is the solution to (5.1), with such a choice of $\Omega^{\prime}, \Omega^{\prime \prime}$. We recall that everywhere both $\operatorname{dist}\left(\Omega^{\prime}, \partial \Omega^{\prime \prime}\right)$ and $\operatorname{dist}\left(\Omega^{\prime \prime}, \partial \Omega\right) \operatorname{depend~on}$ $\operatorname{dist}\left(\Omega^{\prime}, \partial \Omega\right)$ via (2.24). We start from (8.7); as by (1.39) $\delta(q)=q$ when $\theta=p$, we use Poincaré's inequality in order to estimate the left-hand side of (8.7) from below. With $c_{1}$ being the one in (8.7) up to multiplicative constant $c(n, q)$, we have

$$
\begin{aligned}
f_{B_{\varrho}} & \left|u_{k}-\left(u_{k}\right)_{B_{\varrho}}\right|^{q} d x \\
& \leq c_{1}\left\{R^{q-n}\left(\frac{\varrho}{R}\right)^{\beta q / 2} \int_{B_{R}}\left(\left|D u_{k}\right|^{q}+s^{q}\right) d x+\left\|f_{k}\right\|_{L^{1, \theta}\left(B_{R}\right)}^{\frac{q}{p-1}}\right\} .
\end{aligned}
$$

Now, fix $\Omega^{\prime} \subset \subset \Omega^{\prime \prime} \subset \subset \Omega$ as in the proof of Lemma 8.1, and using the same argument used to prove Morrey regularity in the previous proof we find

$$
\left[u_{k}\right]_{B M O\left(\Omega^{\prime}\right)} \leq c\left\|f_{k}\right\|_{L^{1}(\Omega)}^{1 /(p-1)}+c\left\|f_{k}\right\|_{L^{1, \theta}(\Omega)}^{1 /(p-1)}+c s|\Omega|^{1 / q},
$$

with $c \equiv c\left(n, p, L / v, q\right.$, $\left.\operatorname{dist}\left(\Omega^{\prime}, \partial \Omega\right)\right)$. Letting $k \nearrow \infty$, and using of (5.3), we finally obtain

$$
[u]_{B M O\left(\Omega^{\prime}\right)} \leq c[|\mu|(\Omega)]^{1 /(p-1)}+c M^{1 /(p-1)}+c s|\Omega|^{1 / q} .
$$

Now (1.42) is finally proved combining the last estimate with the following trivial consequence of (1.27):

$$
|\mu|(\Omega) \leq[\operatorname{diam}(\Omega)]^{n-\theta} M
$$

and taking into account that $c$ may depend on $\Omega$ too. 
In order to prove the local VMO regularity we assume that $\mu$ satisfies (1.41) locally uniformly in the sense of Definition 2 in Section 2.4. In order to conclude it suffices to prove that: For every $\Omega^{\prime} \subset \subset \Omega$ and every $\varepsilon>0$ there exist $\bar{k} \equiv$ $\bar{k}\left(\varepsilon, \operatorname{dist}\left(\Omega^{\prime}, \partial \Omega\right)\right) \in \mathbb{N}$ and $\bar{\varrho} \equiv \bar{\varrho}\left(\varepsilon, \operatorname{dist}\left(\Omega^{\prime}, \partial \Omega\right)\right) \in \mathbb{N}$, possibly also depending on $n, p, L / v, q, s, \Omega$, such that

$$
f_{B_{\varrho}}\left|u_{k}-\left(u_{k}\right)_{B_{\varrho}}\right|^{q} d x \leq \varepsilon, \quad k \geq \bar{k}, \quad \varrho \leq \bar{\varrho},
$$

whenever $B_{\varrho} \subset \subset \Omega^{\prime \prime}$ is ball centered in $\Omega^{\prime}$. This with (5.6) will finally prove the whole theorem as $\Omega^{\prime} \subset \subset \Omega$ is arbitrary. Using (5.5) and (5.4) with (8.9) we have

$$
\begin{aligned}
& f_{B_{\varrho}}\left|u_{k}-\left(u_{k}\right)_{B_{\varrho}}\right|^{q} d x \\
& \quad \leq c_{1}\left\{R^{q-n}\left(\frac{\varrho}{R}\right)^{\beta q / 2}\left[[|\mu|(\Omega)]^{\frac{q}{p-1}}+s^{q}|\Omega|\right]+\|\mu\|_{L^{1, \theta}\left(B_{R+1 / k}\right)}^{\frac{q}{p-1}}\right\} .
\end{aligned}
$$

Determine a positive radius $\bar{R} \leq \operatorname{dist}\left(\Omega^{\prime}, \partial \Omega^{\prime \prime}\right) / 4$, depending on $\varepsilon$, $\operatorname{dist}\left(\Omega^{\prime}, \partial \Omega\right)$ and on $n, p, L / v, q$, such that $|\mu|\left(B_{r}\right) \leq\left(2 c_{1}\right)^{-1} \varepsilon r^{n-p}$ whenever $r \leq 2 \bar{R}$ and $B_{r} \subset \Omega^{\prime \prime}$. This implies $\|\mu\|_{L^{1, \theta}\left(B_{2 \bar{R}}\right)} \leq\left(2 c_{1}\right)^{-1} \varepsilon$ whenever $B_{2 \bar{R}}$ is centered in $\Omega^{\prime}$. Indeed this and $\bar{R} \leq \operatorname{dist}\left(\Omega^{\prime}, \partial \Omega^{\prime \prime}\right) / 4$ imply $B_{2 \bar{R}} \subset \Omega^{\prime \prime}$. From now on all the balls considered will be centered in $\Omega^{\prime}$. Taking $\bar{k} \equiv \bar{k}\left(\varepsilon, \operatorname{dist}\left(\Omega^{\prime}, \partial \Omega\right)\right) \in \mathbb{N}$, also depending on $n, p, L / \nu, q$, such that $1 / \bar{k} \leq \bar{R}$ we have

$$
c_{1}\|\mu\|_{L^{1, \theta}\left(B_{\bar{R}+1 / k}\right)} \leq \varepsilon / 2 .
$$

This fixes $\bar{k}$ in (8.11). From now on we shall use (8.12) with $R \equiv \bar{R}$. Now take $\bar{\varrho} \equiv \bar{\varrho}\left(\varepsilon, \operatorname{dist}\left(\Omega^{\prime}, \partial \Omega\right)\right) \leq \bar{R}$, also depending on $n, p, L / \nu, q, s, \Omega$, in order to have

$$
c_{1} \bar{R}^{q-n}\left(\frac{\bar{\varrho}}{\bar{R}}\right)^{\beta q / 2}\left[[|\mu|(\Omega)]^{\frac{q}{p-1}}+s^{q}|\Omega|\right] \leq \varepsilon / 2 .
$$

This fixes $\bar{\varrho}$ in (8.11). We finally obtain (8.11) merging (8.13)-(8.14) to (8.12), the latter used with $\bar{R} \equiv R$, and $\varrho \leq \bar{\varrho}$.

Proof of Theorem 1.11. As usual we shall proceed deriving a priori estimates, therefore let $u \in W^{1, p}(\Omega)$ be the solution to (4.2) for a fixed $f \in L^{\infty}(\Omega)$. We shall use the estimates from the proof of Theorem 1.8 below, as explained at the beginning of the section, therefore this proof should be read after the one of Theorem 1.8. Let $B_{R} \subset \subset \Omega$, with $R \leq 1$. By Lemma 2.8 with $q \in(1, m)$

$$
\begin{aligned}
\int_{B_{R}}|D u|^{q} d x & \stackrel{(2.20)}{\leq} m(m-q)^{-1}\left|B_{R}\right|^{1-\frac{q}{m}}\|D u\|_{\mathcal{M}^{m}\left(B_{R}\right)}^{q} \\
& \stackrel{(2.18)}{\leq} c R^{n-\frac{q \theta}{m}}\|D u\|_{\mathcal{M}^{m, \theta}\left(B_{R}\right)}^{q} \\
& \stackrel{(9.35)}{\leq} c\left[\|f\|_{L^{1}(\Omega)}^{\frac{q}{p-1}}+\|f\|_{L^{1, \theta}(\Omega)}^{\frac{q}{p-1}}+s^{q}|\Omega|^{\frac{q}{m}}\right] R^{n-\delta(q)},
\end{aligned}
$$


where $c \equiv c\left(n, p, L / v, q, \operatorname{dist}\left(B_{R}, \partial \Omega\right), \Omega\right)$. We used that $q \theta / m=\delta(q)$, see (1.39). Therefore taking the supremum over all possible such balls with $B_{R} \subset \subset \Omega^{\prime}$ we have

$$
\|D u\|_{L^{q, \delta(q)}\left(\Omega^{\prime}\right)} \leq c\|f\|_{L^{1}(\Omega)}^{1 /(p-1)}+c\|f\|_{L^{1, \theta}(\Omega)}^{1 /(p-1)}+c s|\Omega|^{1 / m},
$$

$c \equiv c\left(n, p, L / v, q, \operatorname{dist}\left(\Omega^{\prime}, \partial \Omega\right), \Omega\right)$. The assertion follows once again via the approximation scheme of Section 5, a lower semicontinuity to handle the left-hand side of the latter estimate, and using (8.10) as for Theorem 1.8.

\section{Marcinkiewicz estimates}

This section contains the proof of Theorem 1.8. One of our starting points here will be the brilliant technique for proving $\mathcal{M}^{n}$-estimates introduced in [27] (case $p=n$, that implies $\theta=p=n$ ). We shall use a delicate combination of some the arguments from the latter paper with the Morrey space estimates of Section 8, a direct comparison argument on certain Calderón-Zygmund type balls, and finally a modification of some ideas from $[14,48]$. A different, elegant approach to $\mathcal{M}^{n}$ estimates based on a suitable version of Gehring's lemma in Marcinkiewicz spaces has been recently given in [44]. Let us emphasize here the fact that our technique is robust enough to catch the borderline case $\theta=p$, and therefore to get the limiting regularity (1.31).

As everywhere else, we shall derive a priori estimates and in the following $u \in W_{0}^{1, p}(\Omega)$ is a solution to (4.2) for a fixed $f \in L^{\infty}(\Omega)$; we assume of course that $\|f\|_{L^{1, \theta}(\Omega)}>0$, otherwise all assertions trivialize. To begin with the proof let us consider two open subsets $\Omega^{\prime} \subset \subset \Omega^{\prime \prime} \subset \subset \Omega$. Take a ball $B_{0}$ with radius $R_{0} \leq 1 / 2$, such that $2 B_{0} \subset \subset \Omega^{\prime \prime}$. We use the restricted maximal function of $f$ relative to $2 B_{0}$, that is

$$
M(f)(x) \equiv M_{2 B_{0}}(f)(x):=\sup _{x \in B, B \subseteq 2 B_{0}} f_{B}|f(y)| d y,
$$

where $B$ is a ball varying amongst all possible ones in $2 B_{0}$. The weak $(1,1)$ estimate

$$
\left|\left\{x \in 2 B_{0}: M_{2 B_{0}}(f)(x)>\lambda\right\}\right| \leq \frac{c(n)}{\lambda} \int_{2 B_{0}}|f(y)| d y \quad \forall \lambda>0,
$$

holds, see for instance [11], and it immediately follows that

$$
\left|\left\{x \in 2 B_{0}: M_{2 B_{0}}(f)(x)>\lambda\right\}\right| \leq \frac{c\|f\|_{L^{1, \theta}\left(2 B_{0}\right)}\left|B_{0}\right|^{1-\theta / n}}{\lambda} \quad \forall \lambda>0 .
$$

Let us fix $R_{0}<t<\varrho<2 R_{0}$. With $\lambda \geq 0$ we shall denote

$$
E_{\lambda}^{t}:=\left\{x \in B_{t}:|D u(x)|>\lambda\right\}, \quad E_{\lambda}^{\varrho}:=\left\{x \in B_{\varrho}:|D u(x)|>\lambda\right\} .
$$


Here the balls $B_{t}, B_{\varrho}$ are concentric to $B_{0}$, and it obviously holds $B_{0} \subset B_{t} \subset B_{\varrho} \subset$ $2 B_{0}$. We recall that $b$ is in (1.9) and $m$ as in (1.28), while in the following $q$ and $q_{1}$ will be fixed numbers such that $p-1 \leq q<q_{1}<b$.

Step 1: Calderón-Zygmund type decomposition. Let us set

$$
\lambda_{0}:=\left(f_{2 B_{0}}\left(|D u|^{q}+s^{q}\right) d x\right)^{\frac{1}{q}},
$$

and from now on we shall always take $\lambda$ large enough to have

$$
\lambda \geq 4^{n / q}(\varrho-t)^{-n / q} \lambda_{0}=: \lambda_{l},
$$

unless otherwise specified. Observe that if $x_{0} \in B_{t}$ then $B\left(x_{0},(\varrho-t) R_{0}\right) \subset B_{\varrho} \subset$ $2 B_{0}$ and therefore

$$
f_{B\left(x_{0},(\varrho-t) R_{0}\right)}\left(|D u|^{q}+s^{q}\right) d x \stackrel{(9.2)}{\leq} 2^{n}(\varrho-t)^{-n} \lambda_{0}^{q} \leq \lambda^{q} ;
$$

in particular

$$
s \leq \lambda
$$

Now, let $x_{0} \in E_{4 \lambda}^{t}$ and define

$$
i\left(x_{0}\right):=\min \left\{i \in \mathbb{N}: f_{B\left(x_{0}, 2^{-i}(\varrho-t) R_{0}\right)}\left(|D u|^{q}+s^{q}\right) d x \geq 4^{q} \lambda^{q}\right\} .
$$

By (9.4) and Lebesgue's differentiation theory for a.e. $x_{0} \in E_{4 \lambda}^{t}$ we have $1 \leq$ $i\left(x_{0}\right)<\infty$, and the family $\left\{B\left(x_{0}, 2^{-i\left(x_{0}\right)}(\varrho-t) R_{0}\right)\right\}$ is a covering of $E_{4 \lambda}^{t}$ up to a negligible set. We may apply Besicovitch covering theorem [5] in order to extract from $\left\{B\left(x_{0}, 2^{-i\left(x_{0}\right)}(\varrho-t) R_{0}\right)\right\}$ a finite number $Q(n)$ of possibly countable families of mutually disjoint balls $\left\{\mathcal{B}_{j}\right\}_{j \leq Q(n)}, \mathcal{B}_{j} \equiv\left\{B_{i}^{j}\right\}$, such that $E_{4 \lambda}^{t}$ is covered by the union of the closure of such balls up to a negligible set. Rename all these balls in order to have a new, possibly countable family $\left\{B_{k}\right\}$. We need to observe that $2 B_{k} \subset B_{Q}$ for every $k$; this follows from the construction, since for a.e. $x_{0} \in B_{0}$ we have $i\left(x_{0}\right) \geq 1$, therefore the radius of $B_{k}$ does not exceed $(\varrho-t) R_{0} / 2$, and being $B_{k}$ centered in $B_{t}$ then $2 B_{k} \subset B_{\varrho}$ follows. All in all, again by construction the following facts hold:

$$
E_{4 \lambda}^{t} \subset \bigcup_{k} \overline{B_{k}} \cup \text { negligible set }, \quad \sum_{k}\left|E_{\lambda}^{\varrho} \cap B_{k}\right| \leq Q(n)\left|E_{\lambda}^{\varrho}\right|, \quad 2 B_{k} \subset B_{\varrho}
$$

and, for every $k \in \mathbb{N}$

$$
4^{q} \lambda^{q} \leq f_{B_{k}}\left(|D u|^{q}+s^{q}\right) d x, \quad f_{2 B_{k}}\left(|D u|^{q}+s^{q}\right) d x<4^{q} \lambda^{q} .
$$


Denote by $R_{k}$ the radius of $B_{k}$, so that $R_{k} \leq R_{0} \leq 1$; using Lemma 8.1 gives

$$
4^{q} \lambda^{q} \leq f_{B_{k}}\left(|D u|^{q}+s^{q}\right) d x \leq c\||D u|+s\|_{L^{q, \delta(q)}\left(2 B_{0}\right)}^{q} R_{k}^{-\frac{q(\theta-1)}{p-1}},
$$

and it follows

$$
R_{k} \leq c K^{\frac{1}{\theta-1}} \lambda^{-\frac{p-1}{\theta-1}}, \quad K:=\||D u|+s\|_{L^{q, \delta(q)}\left(2 B_{0}\right)}^{p-1}+\|f\|_{L^{1, \theta}\left(2 B_{0}\right)} .
$$

Step 2: A density estimate. Here we single out one generic ball $B_{k}$ and argue under the assumption that there exists $x_{k} \in \overline{B_{k}}$ such that

$$
M(f)\left(x_{k}\right) \leq T^{-1} K^{1 /(1-\theta)} \lambda^{m}
$$

with $T \geq 1$ to be determined later. Using Hölder's inequality and the fact that $B_{k} \subset B_{\varrho}$, we start estimating

$$
\begin{aligned}
4^{q} \lambda^{q}\left|B_{k}\right| & \stackrel{(9.6)}{\leq} \int_{B_{k}}\left(|D u|^{q}+s^{q}\right) d x \\
& \stackrel{(9.4)}{\leq} 2 \lambda^{q}\left|B_{k} \backslash E_{\lambda}^{\varrho}\right|+\int_{B_{k} \cap E_{\lambda}^{\varrho}}\left(|D u|^{q}+s^{q}\right) d x \\
& \leq 2 \lambda^{q}\left|B_{k} \backslash E_{\lambda}^{\varrho}\right|+\left(2\left|B_{k} \cap E_{\lambda}^{\varrho}\right|\right)^{1-\frac{q}{q_{1}}}\left(\int_{B_{k} \cap E_{\lambda}^{\varrho}}\left(|D u|^{q_{1}}+s^{q_{1}}\right) d x\right)^{\frac{q}{q_{1}}} .
\end{aligned}
$$

Therefore, another elementary estimation gives

$$
2^{q} \leq \frac{\left|B_{k} \backslash E_{\lambda}^{\varrho}\right|}{\left|B_{k}\right|}+2\left[\frac{\left|B_{k} \cap E_{\lambda}^{\varrho}\right|}{\left|B_{k}\right|}\right]^{1-\frac{q}{q_{1}}} \lambda^{-q}\left(f_{B_{k}}\left(|D u|^{q_{1}}+s^{q_{1}}\right) d x\right)^{\frac{q}{q_{1}}}
$$

We now estimate the last integral. To this aim, let us introduce the comparison function $v_{k} \in u+W_{0}^{1, p}\left(2 B_{k}\right)$ as the unique solution to

$$
\begin{cases}-\operatorname{div} a\left(x, D v_{k}\right)=0 & \text { in } 2 B_{k} \\ v_{k}=u & \text { on } \partial 2 B_{k} .\end{cases}
$$

Now

$$
f_{B_{k}}|D u|^{q_{1}} d x \leq c f_{B_{k}}\left|D u-D v_{k}\right|^{q_{1}} d x+c f_{B_{k}}\left|D v_{k}\right|^{q_{1}} d x
$$


and we estimate the last two integrals. Using Lemma 4.3 we find

$$
\begin{aligned}
f_{2 B_{k}}\left|D u-D v_{k}\right|^{q_{1}} d x & \stackrel{(4.15)}{\leq} c\|f\|_{L^{1, \theta}\left(2 B_{0}\right)}^{\frac{q_{1}-p+1}{p-1}} f_{2 B_{k}}|f| d x R_{k}^{\sigma\left(q_{1}, \theta\right)} \\
& \stackrel{(9.7)}{\leq} c \frac{K^{\frac{1}{\theta-1}}}{\lambda^{m-q_{1}}} f_{2 B_{k}}|f| d x \\
& \stackrel{(9.8)}{\leq} \frac{c \lambda^{q_{1}}}{T},
\end{aligned}
$$

where $c \equiv c\left(n, p, L / v, q_{1}\right)$; in a completely similar way we also get

$$
f_{2 B_{k}}\left|D u-D v_{k}\right|^{q} d x \leq \frac{c \lambda^{q}}{T} .
$$

On the other hand, since $q_{1}<p$, using Hölder's inequality and (3.11), we have

$$
f_{B_{k}}\left|D v_{k}\right|^{q_{1}} d x \leq\left(f_{B_{k}}\left|D v_{k}\right|^{p \chi} d x\right)^{\frac{q_{1}}{p \chi}} \leq c\left(f_{2 B_{k}}\left(\left|D v_{k}\right|^{q}+s^{q}\right) d x\right)^{\frac{q_{1}}{q}} .
$$

In the last line $\chi \equiv \chi(n, p, L / v)>1$ is the higher integrability exponent such that $D v \in L_{\mathrm{loc}}^{p \chi}\left(2 B_{k}, \mathbb{R}^{n}\right)$, that has been determined in Lemma 3.3. In turn, since $T \geq 1$, (9.6) and (9.14) give

$$
f_{2 B_{k}}\left|D v_{k}\right|^{q} d x \leq c f_{2 B_{k}}\left|D u-D v_{k}\right|^{q} d x+c f_{2 B_{k}}|D u|^{q} d x \leq c \lambda^{q} .
$$

Merging (9.16) and (9.15), and using (9.4), gives

$$
f_{2 B_{k}}\left|D v_{k}\right|^{q_{1}} d x \leq c \lambda^{q_{1}} .
$$

Connecting (9.13), (9.17), to (9.12), and using again (9.4), yields

$$
f_{B_{k}}\left(|D u|^{q_{1}}+s^{q_{1}}\right) d x \leq c \lambda^{q_{1}},
$$

where $c \equiv c\left(n, p, L / \nu, q_{1}\right)$. Using this last inequality in (9.10) gives

$$
2^{q} \leq \frac{\left|B_{k} \backslash E_{\lambda}^{\varrho}\right|}{\left|B_{k}\right|}+c_{1}\left[\frac{\left|B_{k} \cap E_{\lambda}^{\varrho}\right|}{\left|B_{k}\right|}\right]^{1-\frac{q}{q_{1}}},
$$

where $c_{1} \equiv c_{1}\left(n, p, L / \nu, q_{1}\right)$, and therefore, since $q_{1}>q$, we have

$$
\frac{\left|B_{k} \cap E_{\lambda}^{\varrho}\right|}{\left|B_{k}\right|} \geq\left[\frac{1}{c_{1}}\left(2^{q}-1\right)\right]^{\frac{q_{1}}{q_{1}-q}}=: \frac{1}{c_{2}}>0,
$$

where $c_{2} \equiv c_{2}\left(n, p, L / v, q, q_{1}\right)$; this is the density estimate we were looking for. 
Step 3: Estimates on balls. We take $H \geq 4^{n / q} \geq 4$ to be chosen, and estimate the measure of $E_{H \lambda}^{t}$ splitting as

$$
\begin{aligned}
\left|E_{H \lambda}^{t}\right| \leq & \left|E_{H \lambda}^{t} \cap\left\{x \in B_{t}: M(f)(x) \leq T^{-1} K^{1 /(1-\theta)} \lambda^{m}\right\}\right| \\
& +\left|E_{H \lambda}^{t} \cap\left\{x \in B_{t}: M(f)(x)>T^{-1} K^{1 /(1-\theta)} \lambda^{m}\right\}\right|=: I+I I .
\end{aligned}
$$

By (9.1), and the definition of $K$ in (9.7), we immediately have

$$
I I \leq c(n) T K^{\frac{\theta}{\theta-1}} \lambda^{-m} R_{0}^{n-\theta}
$$

and we concentrate on $I$. To this aim, since $H \geq 4$ by (9.5) we may estimate

$$
I \leq \sum I_{k}:=\sum\left|E_{H \lambda}^{t} \cap\left\{x \in \overline{B_{k}}: M(f)(x) \leq T^{-1} K^{1 /(1-\theta)} \lambda^{m}\right\}\right|,
$$

and in turn we estimate each $I_{k}$. Fix one; we may assume there exists $x_{k} \in \overline{B_{k}}$ such that (9.8) holds; otherwise $I_{k}=0$ and we are done. By definition of $I_{k}$

$$
\begin{aligned}
I_{k} \leq\left|E_{H \lambda}^{t} \cap \overline{B_{k}}\right| \leq & \left|\left\{x \in \overline{B_{k}}:|D u(x)|>H \lambda\right\}\right| \\
\leq & \left|\left\{x \in \overline{B_{k}}:\left|D u(x)-D v_{k}(x)\right|>H \lambda / 2\right\}\right| \\
& +\left|\left\{x \in \overline{B_{k}}:\left|D v_{k}(x)\right|>H \lambda / 2\right\}\right|=: I I I_{k}+I V_{k} .
\end{aligned}
$$

Then, keeping in mind the definition of $K$ in (9.7)

$$
\begin{aligned}
& I I I_{k} \leq \frac{2^{q}}{H^{q} \lambda^{q}} \int_{B_{k}}\left|D u-D v_{k}\right|^{q} d x \\
& \stackrel{(4.15)}{\leq} \frac{c\|f\|_{L^{1, \theta}\left(2 B_{0}\right)}^{\frac{q-p+1}{p-1}}}{H^{q} \lambda^{q}} \int_{2 B_{k}}|f| d x R_{k}^{\sigma(q, \theta)} \\
& \stackrel{(9.7)}{\leq} \frac{c K^{\frac{q-p+1}{p-1}}}{H^{q} \lambda q} \int_{2 B_{k}}|f| d x R_{k}^{\sigma(q, \theta)} \\
& \stackrel{(9.7)}{\leq} \frac{c K^{\frac{1}{\theta-1}}}{H^{q} \lambda^{m}} \int_{2 B_{k}}|f| d x \\
& \stackrel{(9.8)}{\leq} \frac{c_{3}\left|2 B_{k}\right|}{H^{q} T} \\
& \stackrel{(9.19)}{\leq} \frac{c_{3} c_{2} 2^{n}\left|B_{k} \cap E_{\lambda}^{\varrho}\right|}{H^{q} T}
\end{aligned}
$$


Let $\chi \equiv \chi(n, p, L / v)>1$ be as in (9.15), that is the number determined in Lemma 3.3; using (9.4) we have

$$
\begin{aligned}
I V_{k} & \leq \frac{2^{p \chi}}{H^{p \chi} \lambda^{p \chi}} \int_{B_{k}}\left|D v_{k}\right|^{p \chi} d x \\
& \stackrel{(3.11)}{\leq} \frac{c\left|2 B_{k}\right|}{H^{p \chi} \lambda^{p \chi}}\left(\int_{2 B_{k}}\left(\left|D v_{k}\right|^{q}+s^{q}\right) d x\right)^{\frac{p \chi}{q}} \\
& \stackrel{(9.16),(9.4)}{\leq} \frac{c_{4}\left|2 B_{k}\right|}{H^{p \chi}} \\
& \stackrel{c_{4} c_{2} 2^{n}\left|B_{k} \cap E_{\lambda}^{\varrho}\right|}{H^{p \chi}} .
\end{aligned}
$$

Connecting the estimates found for $I I I_{k}, I V_{k}$ to (9.23) gives

$$
I_{k} \leq c_{5}\left[H^{-q} T^{-1}+H^{-p \chi}\right]\left|B_{k} \cap E_{\lambda}^{\varrho}\right|,
$$

with $c_{5} \equiv c_{5}\left(n, p, L / v, q, q_{1}\right)$. Summing up on $k$ using (9.22), (9.5) yields

$$
I \leq c_{5} Q(n)\left[H^{-q} T^{-1}+H^{-p \chi}\right]\left|E_{\lambda}^{\varrho}\right| .
$$

Merging the latter estimate and (9.21) with (9.20) we finally have

$$
\left|E_{H \lambda}^{t}\right| \leq c_{6}\left[H^{-q} T^{-1}+H^{-p \chi}\right]\left|E_{\lambda}^{\varrho}\right|+c_{6} T K^{\frac{\theta}{\theta-1}} \lambda^{-m} R_{0}^{n-\theta},
$$

where $c_{6} \equiv c_{6}\left(n, p, L / \nu, q, q_{1}\right)$, while $H \geq 4^{n / q}$ and $T \geq 1$ are still to be chosen.

Step 4: Iteration and a priori estimate. Let us for a moment assume that $R_{0}=$ $1 / 2$; we shall eventually deal with the general case by means of a scaling argument. We introduce the level function $l(\cdot, \cdot)$ as

$$
l(\lambda, \gamma):=\lambda^{m}\left|E_{\lambda}^{\gamma}\right|, \quad \text { for every } \gamma \in[1 / 2,1] \text {, and } \lambda>0,
$$

and observe that (9.24) can be rephrased as

$$
l(H \lambda, t) \leq c_{6}\left[H^{m-q} T^{-1}+H^{m-p \chi}\right] l(\lambda, \varrho)+c_{6} H^{m} T K^{\frac{\theta}{\theta-1}} .
$$

Now observe that $m \leq p<p \chi$, and equality in the first inequality occurs iff $p=\theta$; therefore we take $H$ large enough in order to have $c_{6} H^{m-p \chi} \leq 1 / 4$; taking into account the dependence of $m, \chi$, and $c_{6}$, this fixes $H \equiv H\left(n, p, L / \nu, q, q_{1}\right)$. Next, take $T$ large enough to balance $H$ i.e. $T:=4 c_{6} H^{m-q}$, recall that $m>q$; therefore $T \equiv T\left(n, p, L / v, q, q_{1}\right)$. With such choices (9.26) gives

$$
l(H \lambda, t) \leq(1 / 2) l(\lambda, \varrho)+c_{7} K^{\frac{\theta}{\theta-1}},
$$


with $c_{7} \equiv c_{7}\left(n, p, L / \nu, q, q_{1}\right)$. The last inequality holds whenever $\lambda$ satisfies (9.3), therefore, taking into account the definition in (2.17) to bound the right-hand side, with $\lambda_{l}$ as in (9.3) we have

$$
\sup _{\lambda \geq \lambda_{l}} l(H \lambda, t) \leq(1 / 2)\|D u\|_{\mathcal{M}^{m}\left(B_{\varrho}\right)}^{m}+c_{7} K^{\frac{\theta}{\theta-1}}
$$

and obviously, again by the definition in (2.17), and (9.2)-(9.3), we have

$$
\|D u\|_{\mathcal{M}^{m}\left(B_{t}\right)}^{m} \leq(1 / 2)\|D u\|_{\mathcal{M}^{m}\left(B_{\varrho}\right)}^{m}+H^{m}\left|2 B_{0}\right|(\varrho-t)^{-n m / q} \lambda_{0}^{m}+c_{7} K^{\frac{\theta}{\theta-1}} .
$$

Observe also that we are proving a priori estimates for approximate solutions, and therefore we are assuming that $u \in W^{1, p}$; since $m \leq p$ in any case it follows that $\|D u\|_{\mathcal{M}^{m}\left(2 B_{0}\right)}$ is finite. We can apply Lemma 2.12 with $\varphi(t):=\|D u\|_{\mathcal{M}^{m}\left(B_{t}\right)}^{m}$, $R_{0}=1 / 2$, and $1 / 2<t<\varrho<1$; this yields

$$
\|D u\|_{\mathcal{M}^{m}\left(B_{0}\right)}^{m} \leq c \lambda_{0}^{m}+c K^{\frac{\theta}{\theta-1}}
$$

with $c \equiv c\left(n, p, L / \nu, q, q_{1}\right)$, as $H$ depends on $n, p, L / \nu, q, q_{1}$ and $\left|2 B_{0}\right| \leq c(n)$. Using the definition of $\lambda_{0}$ in (9.2) and that $R_{0}=1 / 2$, we observe that

$$
\lambda_{0}^{m} \leq\left(f_{2 B_{0}}\left(|D u|^{q}+s^{q}\right) d x\right)^{\frac{m}{q}} \leq c\||D u|+s\|_{L^{q, \delta(q)}\left(2 B_{0}\right)}^{m} .
$$

Merging (9.29) with (9.28), taking into account the definition of $K$ in (9.7) we easily obtain

$$
\||D u|+s\|_{\mathcal{M}^{m}\left(B_{0}\right)}^{m} \leq c\left[\||D u|+s\|_{L^{q, \delta(q)}\left(2 B_{0}\right)}^{m}+\|f\|_{L^{1, \theta}\left(2 B_{0}\right)}^{\frac{m}{p-1}}\right] .
$$

All this holds provided $R_{0}=1 / 2$. The general case $R_{0} \in(0,1 / 2]$ can be dealt with by scaling, that is: first considering a general ball $B_{0}$, then from the very beginning of the proof reducing the problem to the case $R_{0}=1 / 2$ scaling as in (4.5), then obtaining (9.30) for the scaled solution $\tilde{u}$ with data $\tilde{f}$, and finally scaling back to $u$; then using also Lemma 2.5 all this yields, for any $R_{0} \in(0,1 / 2]$

$$
\||D u|+s\|_{\mathcal{M}^{m}\left(B_{0}\right)}^{m} \leq c\left[\||D u|+s\|_{L^{q, \delta(q)}\left(2 B_{0}\right)}^{m}+\|f\|_{L^{1, \theta}\left(2 B_{0}\right)}^{\frac{m}{p-1}}\right] R_{0}^{n-\theta}
$$

where again $c \equiv c\left(n, p, L / \nu, q, q_{1}\right)$. For later convenience let us observe that taking $q=p-1$ in the latter estimate, and using that $\delta(p-1)=\theta-1$ by (1.39), we have

$$
\||D u|+s\|_{\mathcal{M}^{m}\left(B_{0}\right)}^{m} \leq c\left[\||D u|+s\|_{L^{p-1, \theta-1}\left(2 B_{0}\right)}^{m}+\|f\|_{L^{1, \theta}\left(2 B_{0}\right)}^{\frac{m}{p-1}}\right] R_{0}^{n-\theta} .
$$


Using (9.31) together with a standard covering argument, and an elementary estimation involving the definition in (2.18), we have

$$
\||D u|+s\|_{\mathcal{M}^{m, \theta}\left(\Omega^{\prime}\right)} \leq c\||D u|+s\|_{L^{q, \delta(q)}\left(\Omega^{\prime \prime}\right)}+c\|f\|_{L^{1, \theta}\left(\Omega^{\prime \prime}\right)}^{1 /(p-1)},
$$

where $c \equiv c\left(n, p, L / v, q, \Omega^{\prime}, \Omega^{\prime \prime}\right)$, since (2.24) holds. Finally using (8.1) in the previous estimate, and via Lemma 2.13 passing again to outer and inner open subsets to $\Omega^{\prime}$ and $\Omega^{\prime \prime}$ respectively, as everywhere open subsets $\Omega^{\prime}, \Omega^{\prime \prime}$ are arbitrary, we conclude with the desired local a priori estimate

$$
\||D u|+s\|_{\mathcal{M}^{m, \theta}\left(\Omega^{\prime}\right)} \leq c\||D u|+s\|_{L^{q}\left(\Omega^{\prime \prime}\right)}+c\|f\|_{L^{1, \theta}\left(\Omega^{\prime \prime}\right)}^{1 /(p-1)},
$$

for any choice $\Omega^{\prime} \subset \subset \Omega^{\prime \prime}$, where $c \equiv c\left(n, p, L / v, q, \Omega^{\prime}, \Omega^{\prime \prime}\right)$. In turn, with $\Omega^{\prime} \subset \subset$ $\Omega$ fixed as in the statement of Theorem 1.8, we can pick $\Omega^{\prime \prime}$ in (9.34) as prescribed in Lemma 2.13, and taking into account (4.25) we get

$$
\||D u|+s\|_{\mathcal{M}^{m, \theta}\left(\Omega^{\prime}\right)} \leq c\|f\|_{L^{1}(\Omega)}^{1 /(p-1)}+c\|f\|_{L^{1, \theta}(\Omega)}^{1 /(p-1)}+c s|\Omega|^{1 / q},
$$

where now $c \equiv c\left(n, p, L / v, q, \Omega^{\prime}, \Omega\right)$. Applying the latter inequality to $u_{k}$ from (5.1) and taking into account the approximation scheme of Section 5, and in particular (5.3), as in the proof of Theorem 1.2 we get

$$
\||D u|+s\|_{\mathcal{M}^{m, \theta}\left(\Omega^{\prime}\right)} \leq c[|\mu|(\Omega)]^{1 /(p-1)}+c M^{1 /(p-1)}+c s|\Omega|^{1 / q},
$$

where now $u$ is the solution to the original problem (1.1) constructed in Section 5. The assertion of Theorem 1.8 with estimate (1.30) follow plugging estimate (8.10) in (9.36). Just one remark about the convergence of the approximating solutions $u_{k}$. In the proof of Theorem 1.2 we used the higher (fractional) differentiability of solutions to pass to the limit via compactness; this information is not available here since in Theorem 1.8 we are just assuming a measurable dependence of the coefficients, and not (1.4), which was in turn necessary to get differentiability of $D u$. In the present case the converge of the solutions $u_{k}$ can be nevertheless obtained exactly as in $[7,8,26]$.

Remark 9.1 (A local estimate). Estimate (1.30) has a local companion. More precisely, having (9.34) at our disposal, we may apply the usual scaling procedure in (4.5), as already done for instance to obtain (1.16). Using such estimates for the approximating problems (5.1), and employing Lemma 2.5 we end up with the natural estimate

$$
\left\|\left|D u_{k}\right|+s\right\|_{\mathcal{M}^{m, \theta}\left(B_{R / 2}\right)} \leq c R^{\frac{\theta-1}{p-1}-\frac{n}{q}}\left\|\left|D u_{k}\right|+s\right\|_{L^{q}\left(B_{R}\right)}+c\left\|f_{k}\right\|_{L^{1, \theta}\left(B_{R}\right)}^{1 /(p-1)},
$$

for $q \in[p-1, b)$ and $c \equiv c(n, p, L / v)$, whenever $B_{R} \subset \subset \Omega$. Using (5.4)-(5.5), and letting $k \nearrow \infty$ we conclude with

$$
\||D u|+s\|_{\mathcal{M}^{m, \theta}\left(B_{R / 2}\right)} \leq c R^{\frac{\theta-1}{p-1}-\frac{n}{q}}\||D u|+s\|_{L^{q}\left(B_{R}\right)}+c\|\mu\|_{L^{1, \theta}\left(B_{R+\varepsilon}\right)}^{1 /(p-1)}, \quad \forall \varepsilon>0 .
$$


Remark 9.2 (On the limit case $\theta=p$ ). In proving Theorem 1.8 we used (3.11) from Lemma 3.3 to estimate $I V_{k}$. In turn Lemma 3.3 uses Gehring's lemma. The use of Gehring's lemma is actually needed only in the borderline case $\theta=p$, when $m=p$. Indeed in (9.26) we need $m-p \chi<0$. Now observe that $m<p$ as soon as $\theta<p$. Therefore in this latter case we may use inequality (3.11) with $p$ replacing $p \chi$; in (9.26) we would have $H^{m-p}$, still small taking $H$ large. All this does not need Gehring's lemma: indeed for solutions to (3.3) the basic Caccioppoli's type inequality, together with Poincaré's one (see [34], Chapters 6-7) give

$$
\left(f_{B_{R / 2}}|D v|^{p} d x\right)^{\frac{1}{p}} \leq c\left(f_{B_{R}}\left(|D v|^{\frac{n p}{n+p}}+s^{\frac{n p}{n+p}}\right) d x\right)^{\frac{n+p}{n p}} .
$$

From this the new form of (3.11), with $p$ replacing $p \chi$, follows by Lemma 3.1.

\section{The super-capacitary case}

In this section we are going to prove Theorem 1.9. As usual we shall derive a priori estimates; in the following let $u \in W_{0}^{1, p}(\Omega)$ be the solution to (4.2) for a fixed $f \in L^{\infty}(\Omega)$. Take $B_{4 R} \subset \subset \Omega$ with $4 R \leq 1$, and then scale $u(x)$ in $B_{R}$ as in (4.5), therefore obtaining a solution $\tilde{u}(y)$ in $B_{1}$. We fix $d \in(0,1)$. Now apply (6.40) with $\Omega^{\prime} \equiv B_{1 / 2}$ and $q=p-1$ to have

$$
\begin{aligned}
\sup _{h} \int_{B_{1 / 2}} \frac{\left|\tau_{i, h} D \tilde{u}(y)\right|^{p-1}}{|h|^{1-d}} d y & \leq c\||D \tilde{u}|+s\|_{L^{p-1}\left(B_{1}\right)}^{p-1}+c\|\tilde{f}\|_{L^{1}\left(B_{1}\right)} \\
& \leq c\||D \tilde{u}|+s\|_{L^{p-1, \theta-1}\left(B_{1}\right)}^{p-1}+c\|\tilde{f}\|_{L^{1, \theta}\left(B_{1}\right)},
\end{aligned}
$$

for every $i \in\{1, \ldots, n\}$, where $0<|h|<1 / 4$ and $c \equiv c(n, p, L / \nu, d)$. We have used that $\sigma(p-1, \theta)=1$ and $\delta(p-1)=\theta-1$ for every $\theta \in[p, n]$, by (4.1) and (1.39) respectively; recall also (6.2). Notice that the application of (6.40) to $\tilde{u}$ is legitimate since the arguments for Lemma 6.3 are local, making no use of boundary information for the solution. Scaling back (10.1) to $B_{R}$ via Lemma 2.5 gives

$$
\sup _{h} \int_{B_{R / 2}} \frac{\left|\tau_{i, h} D u(x)\right|^{p-1}}{|h|^{1-d}} d x \leq c M_{p-1}^{p-1}\left(B_{R}\right) R^{n-\theta+d},
$$

where $c \equiv c(n, p, L / \nu, d)$, for every $i \in\{1, \ldots, n\}$ where $0<|h|<R / 4$, and where

$$
M_{q}\left(B_{R}\right):=\||D u|+s\|_{L^{q, \delta(q)}\left(B_{R}\right)}+\|f\|_{L^{1, \theta}\left(B_{R}\right)}^{1 /(p-1)}, \quad q \in[p-1, m) .
$$

Now take $q \in(p-1, m)$ and select $\gamma \in(0,1)$ such that

$$
q=\frac{(\theta-\gamma)(p-1)}{\theta-1} \quad \Longleftrightarrow \quad \sigma(q, \theta)=\gamma .
$$


If $\gamma_{0} \in(0, \gamma)$ then

$$
q<m_{0}:=\frac{\left(\theta-\gamma_{0}\right)(p-1)}{\theta-1}<m,
$$

and write, with $t \in(0,1)$

$$
q=(1-t)(p-1)+t m_{0}=\left(\frac{\gamma-\gamma_{0}}{1-\gamma_{0}}\right)(p-1)+\left(\frac{1-\gamma}{1-\gamma_{0}}\right) m_{0} .
$$

It follows

$$
\frac{m-m_{0}}{m}=\frac{\gamma_{0}}{\theta}, \quad \frac{m_{0}}{m}=\frac{\theta-\gamma_{0}}{\theta} .
$$

Now, by estimate (9.32) we have

$$
\||D u|+s\|_{\mathcal{M}^{m}\left(B_{R}\right)} \leq c\left[\||D u|+s\|_{L^{p-1, \theta-1}\left(B_{2 R}\right)}+\|f\|_{L^{1, \theta}\left(B_{2 R}\right)}^{\frac{1}{p-1}}\right] R^{\frac{n-\theta}{m}} .
$$

Using Lemma 2.8, the latter estimate and (10.7) we find

$$
\begin{aligned}
& \int_{B_{R}}|D u|^{m_{0}} d x \leq m\left(m-m_{0}\right)^{-1} R^{n-\frac{m_{0} n}{m}}\|D u\|_{\mathcal{M}^{m}\left(B_{R}\right)}^{m_{0}} \\
& \leq c \gamma_{0}^{-1} R^{n-\frac{m_{0} n}{m}+\frac{m_{0}}{m}(n-\theta)}\left[\||D u|+s\|_{L^{p-1, \theta-1}\left(B_{2 R}\right)}^{m_{0}}+\|f\|_{L^{1, \theta}\left(B_{2 R}\right)}^{\frac{m_{0}}{p-1}}\right] \\
& \leq c \gamma_{0}^{-1} R^{n-\frac{m_{0} \theta}{m}} M_{p-1}^{m_{0}}\left(B_{2 R}\right)=c R^{n-\theta+\gamma_{0}} M_{p-1}^{m_{0}}\left(B_{2 R}\right)
\end{aligned}
$$

where $c \equiv c\left(n, p, L / v, \gamma_{0}\right)$. Now, by (10.6) and Hölder's inequality

$$
\begin{gathered}
\int_{B_{R / 2}}\left|\tau_{i, h} D u(x)\right|^{q} d x=\int_{B_{R / 2}}\left|\tau_{i, h} D u(x)\right|^{(1-t)(p-1)}\left|\tau_{i, h} D u(x)\right|^{t m_{0}} d x \\
\leq\left(\int_{B_{R / 2}}\left|\tau_{i, h} D u(x)\right|^{p-1} d x\right)^{1-t}\left(\int_{B_{R / 2}}\left|\tau_{i, h} D u(x)\right|^{m_{0}} d x\right)^{t} \\
\leq|h|^{(1-d)(1-t)}\left(\int_{B_{R / 2}} \frac{\left|\tau_{i, h} D u(x)\right|^{p-1}}{|h|^{1-d}} d x\right)^{1-t} \\
\cdot\left(\int_{B_{R / 2}}\left(|D u(x)|^{m_{0}}+\left|D u\left(x+h e_{i}\right)\right|^{m_{0}}\right) d x\right)^{t} .
\end{gathered}
$$

In turn, taking $|h| \leq R / 4$, and using (10.8) we have

$$
\begin{aligned}
\int_{B_{R / 2}}\left(|D u(x)|^{m_{0}}+\left|D u\left(x+h e_{i}\right)\right|^{m_{0}}\right) d x & \leq 2 \int_{B_{R}}|D u|^{m_{0}} d x \\
& \leq c M_{p-1}^{m_{0}}\left(B_{2 R}\right) R^{n-\theta+\gamma_{0}} .
\end{aligned}
$$


Combining (10.2), (10.9) and (10.10), and taking into account (10.6) we have

$$
\sup _{h} \int_{B_{R / 2}} \frac{\left|\tau_{i, h} D u(x)\right|^{q}}{|h|^{(1-d)(1-t)}} d x \leq c M_{p-1}^{q}\left(B_{2 R}\right) R^{n-\theta+(1-t) d+t \gamma_{0}},
$$

where $c \equiv\left(n, p, L / v, d, \gamma_{0}\right)$, and $h$ is a real number such that $0<|h|<R / 4$. Since $i \in\{1, \ldots, n\}$ is arbitrary the last inequality yields in a standard way

$$
\sup _{h} \int_{B_{R / 2}} \frac{|D u(x+h)-D u(x)|^{q}}{|h|^{\sigma}} d x \leq c M_{p-1}^{q}\left(B_{2 R}\right) R^{n-\theta+(1-t) d+t \gamma_{0}},
$$

where this time $h \in \mathbb{R}^{n}$ with $|h| \in(0, R / 4]$. Here we have set

$$
\sigma:=(1-d)(1-t) \stackrel{(10.4),(10.6)}{=}(1-d)\left(\frac{\sigma(q, \theta)-\gamma_{0}}{1-\gamma_{0}}\right) \text {. }
$$

As $\sigma(q, \theta)=\gamma$ by (10.4), a direct computation reveals that $(1-t) d+t \gamma_{0}>$ $d \sigma(q, \theta)$; using that $R \leq 1$ and the latter inequality in (10.11) we have

$$
\sup _{h} \int_{B_{R / 2}} \frac{|D u(x+h)-D u(x)|^{q}}{|h|^{\sigma}} d x \leq c M_{p-1}^{q}\left(B_{2 R}\right) R^{n-\theta+d \sigma(q, \theta)},
$$

with $\sigma$ as in (10.12) and $c \equiv c\left(n, p, L / v, d, \gamma_{0}\right)$. Estimate (10.13) has been proved for $q \in(p-1, m)$. It actually holds for the case $q=p-1$ too, and even with $\gamma_{0}=0$ in (10.12). This is just a consequence of $\sigma(p-1, \theta)=1$ and (10.2). We are now ready to conclude the proof. Take $\gamma_{1} \in(0, \sigma)$; then changing variables

$$
\begin{aligned}
& \int_{B_{R / 2}} \int_{B_{R / 2}} \frac{|D u(x)-D u(y)|^{q}}{|x-y|^{n+\sigma-\gamma_{1}}} d x d y \\
& \leq \int_{B(0, R)} \frac{1}{|h|^{n-\gamma_{1}}} \int_{B_{R / 2}} \frac{|D u(x+h)-D u(x)|^{q}}{|h|^{\sigma}} d x d h \\
& \leq\left(\int_{B(0, R)} \frac{d h}{|h|^{n-\gamma_{1}}}\right) \sup _{h} \int_{B_{R / 2}} \frac{|D u(x+h)-D u(x)|^{q}}{|h|^{\sigma}} d x \\
& \stackrel{(10.13)}{\leq} c(n) \gamma_{1}^{-1} M_{p-1}^{q}\left(B_{2 R}\right) R^{n-\theta+d \sigma(q, \theta)},
\end{aligned}
$$

valid for any $q \in[p-1, m)$, where $c \equiv c\left(n, p, L / v, \gamma_{0}\right)$. Therefore since in (10.12) and (10.14) $\gamma_{0}, \gamma_{1}$ can be picked arbitrarily small, all in all we have proved that

$$
[D u]_{\sigma / q, q ; B_{R}} \leq c M_{p-1}^{q}\left(B_{4 R}\right) R^{n-\theta+d \sigma(q, \theta)}, \quad \sigma<(1-d) \sigma(q, \theta),
$$

for all balls $B_{R}$ such that $B_{4 R} \subset \subset \Omega$, and $q \in[p-1, m)$. The constant $c$ depends on $n, p, L / \nu, q, d, \sigma$. This needs an explanation. The constant $c$ blows up when 
$q \nearrow m$ and/or $\sigma \nearrow(1-d) \sigma(q, \theta)$. Indeed, taking $q$ close to $m$ forces $\gamma_{0}$ to be small via (10.5), and this in turn increases $c$ via (10.8); on the other hand taking $\sigma$ close to $(1-d) \sigma(q, \theta)$ forces $\gamma_{0}, \gamma_{1}$ to be small via (10.12),(10.14) respectively, and this again increases $c$ via (10.8) and (10.14).

Now using (10.15) together with a standard covering argument, and the fact that $d$ can be chosen arbitrarily small, and taking into account the definition of $M_{q}\left(B_{R}\right)$ in (10.3), we conclude that for every couple of open subsets $\Omega^{\prime} \subset \subset \Omega^{\prime \prime} \subset$ $\subset \Omega$ and every $\sigma<\sigma(q, \theta)$ it holds

$$
\|D u\|_{W^{\sigma / q, q, \theta}\left(\Omega^{\prime}\right)} \leq c\||D u|+s\|_{L^{p-1, \theta-1}\left(\Omega^{\prime \prime}\right)}+c\|f\|_{L^{1, \theta}\left(\Omega^{\prime \prime}\right)}^{1 /(p-1)} .
$$

Finally using (8.2) with $q=p-1$, and changing subsets via Lemma 2.13 we gain

$$
\|D u\|_{W^{\sigma / q, q, \theta}\left(\Omega^{\prime}\right)} \leq c\|f\|_{L^{1}(\Omega)}^{1 /(p-1)}+c\|f\|_{L^{1, \theta}(\Omega)}^{1 /(p-1)}+c s|\Omega|^{1 / q},
$$

and the constant depends on $n, p, L / v, q, \sigma, \operatorname{dist}\left(\Omega^{\prime}, \partial \Omega\right)$. The assertion of Theorem 1.9, together with estimate (1.35) follow via the approximation scheme of Section 5 as for the other proofs of this paper.

Remark 10.1 (Fractional differentiability vs Morrey regularity). Let us go back to (10.15), keep now $d$ fixed in $(0,1)$, not necessarily "small" in order to approach $\sigma(q, \theta)$ with $\sigma$. Then, again via the approximation of Section 5, it easily follows

$$
D u \in W_{\mathrm{loc}}^{\sigma / q, q, \theta+d \sigma(q, \theta)}\left(\Omega, \mathbb{R}^{n}\right), \quad \text { for every } \sigma<(1-d) \sigma(q, \theta) .
$$

With $q \in[p-1, m)$ being fixed, inclusion (10.18) tells us that if we decrease the rate of differentiability down to $(1-d) \sigma(q, \theta)$, we gain in the Morrey scale up to $\theta+d \sigma(q, \theta)$. Observe that inclusion (10.18) realizes a perfect interpolation between the maximal differentiability in (1.32) that we may obtain taking $d$ close to 0 , and the maximal Morrey regularity in (1.40) that we may obtain formally letting $d \nearrow 1$ in (10.18), as $\theta+d \sigma(q, \theta) \nearrow \delta(q)$ when $d \nearrow 1$; look at (1.39) and (4.1). In other words, with a very rough but suggestive notation

$$
\lim _{d \searrow 0} W^{(1-d) \sigma(q, \theta) / q, q, \theta+d \sigma(q, \theta)}=W^{\sigma(q, \theta) / q, q, \theta},
$$

and

$$
\lim _{d \nearrow 1} W^{(1-d) \sigma(q, \theta) / q, q, \theta+d \sigma(q, \theta)}=L^{q, \delta(q)} .
$$

More in general, since when considering Morrey decay properties as (1.27) the exponent $\theta$ replaces $n$ everywhere, the integer dimension of the space $W^{\alpha, q, \theta}$ should be defined as $\alpha-\theta / q$, compare with Remark 6.4. In this respect, exactly as in Remark 6.4, all the spaces $W^{(1-d) \sigma(q, \theta) / q, q, \theta+d \sigma(q, \theta)}$ share the same integer dimension $(\theta-1) /(p-1)$, for every possible choice of $q \in[p-1, m)$ and $d \in(0,1)$. 


\section{Sharpness, comparisons, extensions}

We hereby discuss the sharpness of some of the foregoing results, and outline a few extensions and connections.

\subsection{Sharpness of Theorem 1.2}

The result in (1.14) is sharp for every choice of the couple $(q, \sigma(q))$ in the range (1.15), and in particular the inclusions (1.12) and (1.13) are sharp too. Indeed, we cannot have $D u \in W_{\mathrm{loc}}^{\sigma(q) / q, q}$, as shown by the usual counterexample [45]. Consider the equation (1.6) in the ball $B_{1} \equiv \Omega$, with $\mu \equiv \delta$, the Dirac measure charging the origin, with the related zero-Dirichlet condition. The unique solution to problem (1.1) is now given by the Green's function

$$
u(x):=c(n, p)\left\{\begin{array}{cll}
|x|^{\frac{p-n}{p-1}}-1 & \text { if } & p<n \\
\log |x| & \text { if } & p=n
\end{array}\right.
$$

where $c(n, p)$ is a suitable re-normalization constant. We have $D u \in \mathcal{M}^{b}\left(B_{1}\right)$, but $D u \notin L_{\text {loc }}^{b}\left(B_{1}\right)$, and crucial integrability is lost at the origin. Now, assume by contradiction that $D u \in W_{\mathrm{loc}}^{\sigma(q) / q, q}\left(B_{1}\right)$, then by Theorem 2.2 we would have $D u \in L_{\text {loc }}^{n q /(n-\sigma(q))}\left(B_{1}\right)$, but this is impossible since $n q /(n-\sigma(q))=b$ by (1.15). Therefore $D u \notin W_{\mathrm{loc}}^{\sigma(q) / q, q}\left(B_{1}\right)$, and this gives the optimality of Theorem 1.2. On the other hand, as $n q /(n-\sigma(q))=b$, then assuming Theorem 1.2 allows to recover the original integrability result in (1.8) in a local form, again via Theorem 2.2.

\subsection{About Theorem 1.4.}

This is also sharp. In fact assuming that

$$
V(D u) \in W_{\mathrm{loc}}^{\frac{p}{2(p-1)}, \frac{2(p-1)}{p}}\left(\Omega, \mathbb{R}^{n}\right), \quad \text { for every } \varepsilon \in(0,1)
$$

by the fractional Sobolev embedding theorem 2.2 we would get

$$
V(D u) \in L_{\text {loc }}^{\frac{n(p-1) 2}{(n-1) p}}\left(\Omega, \mathbb{R}^{n}\right),
$$

and in turn this would imply, via (2.3), that

$$
D u \in L_{\operatorname{loc}}^{\frac{n(p-1)}{n-1}}\left(\Omega, \mathbb{R}^{n}\right),
$$

which is excluded by the discussion of Section 11.1. Theorem 1.4 can be regarded as a non-linear version of the so called "uniformization of singularities principle", well-known in Complex Analysis: raising a function to a suitably large power we 
get a function with better regularity properties. In such respect we conclude with an open problem that for the sake of simplicity we state for solutions to equations involving the $p$-Laplacean operator (1.6). Take $\gamma \in \mathbb{R}$ such that

$$
\frac{p-2}{2} \leq \gamma \leq p-2
$$

and prove - or disprove - in the spirit of Theorem 1.4, that, once a Dirichlet class is fixed as boundary datum, there exists a SOLA solution to (1.6) such that

$$
|D u|^{\gamma} D u \in W_{\mathrm{loc}}^{\frac{\gamma+1}{p-1}-\varepsilon, \frac{p-1}{\gamma+1}}\left(\Omega, \mathbb{R}^{n}\right), \quad \text { for every } \varepsilon>0 .
$$

In the first limit case $\gamma=(p-2) / 2$ this is essentially the content of Theorem 1.4 , while in the other borderline case $\gamma=p-2$ this amounts to prove that $|D u|^{p-2} D u \in W_{\text {loc }}^{1-\varepsilon, 1}\left(\Omega, \mathbb{R}^{n}\right)$, for every $\varepsilon>0$. When $p=2$ all such statements collapse in Theorem 1.2. Observe that, exactly as for (1.21), for every choice of $\gamma$ in the range (11.1) the product between the differentiability and the integrability indexes in (11.2) remains constant, up to the presence of $\varepsilon$.

\subsection{The exponent $m$ in (1.28)}

We now demonstrate the optimality of $m$ in (1.29) in the case $p=2$ by comparing Theorem 1.8 with the optimal ones of Adams [2] for the case $\Delta u=\mu$. Since our results are local, up to a standard localization procedure we shall consider the latter equation in the whole $\mathbb{R}^{n}$. We consider the fractional integral operator defined by

$$
I_{\alpha}(\mu)(x):=\int_{\mathbb{R}^{n}} \frac{d \mu(y)}{|x-y|^{n-\alpha}}, \quad \alpha \in(0, n] .
$$

When $\mu$ has compact support, the unique solution to $\Delta u=\mu$ is given by $u(x):=$ $c_{1} I_{2}(\mu)(x)$, with $c$ being a suitable re-normalization constant; as a consequence $D u(x)=c_{2} I_{1}(\mu)(x)$; see also [54]. Now we recall the following result of Adams [2]:

$$
I_{\alpha}: L^{1, \theta} \rightarrow \mathcal{M}^{\theta /(\theta-\alpha), \theta},
$$

that is sharp in the sense that we cannot expect $I_{\alpha}(\mu) \in L^{\theta /(\theta-\alpha)}$, even locally, for $\mu \in L^{1, \theta}$, see [2] page 770, no. 2. Taking in our case $\alpha=1$ gives $\theta /(\theta-\alpha)=m$, and therefore the exponent $m$ is the natural one for $p=2$.

The case $p>2$ cannot be treated by such an argument since no explicit representation formula is available for solutions to (1.6). We just remark that in the case $p>2$ the exponent $m$ is obtained by multiplying the one for $p=2$ times $(p-1)$. This appears to be a natural phenomenon for measure data problems [9]. We hereby conjecture that the exponent $m$ is optimal for every $p>2$. Finally observe how the fact that $\theta$ replaces $n$ everywhere when assuming (1.27) is in perfect accordance with the embedding properties for Sobolev-Morrey spaces. Indeed, assuming 
$D u \in L^{p, \theta}$ with $p<\theta$, leads to the improved embedding $u \in L^{\theta p /(\theta-p)}[2,15,16]$; this covers the usual Sobolev embedding theorem when $\theta=n$.

We remark that in order to prove Theorem 1.8 the full strength of the assumptions (1.2) is not needed. Indeed, as a careful inspection of the proof reveals, it is sufficient to use the assumption in the first line of (1.2) together with the first inequality in (3.9).

\subsection{Sharpness of Theorem 1.9}

Here we discuss the optimality of the choice of the couple $(q, \sigma(q, \theta))$ in (1.32) in the range displayed in (1.33). The input here in the Sobolev-Morrey embedding Theorem in the fractional case. We have that $W^{\alpha, q, \theta}$ embeds in $L^{t}$ for every $t<$ $\theta q /(\theta-\alpha q)$ whenever $\alpha q<\theta$; see for instance [64]. Now take $p=2$ and assume that $D u \in W_{\mathrm{loc}}^{(\sigma(q, \theta)+\varepsilon) / q, q}$ for some $\varepsilon>0$; since $m=\theta q /(\theta-\sigma(q, \theta))$ we would conclude with $D u \in L_{\mathrm{loc}}^{m}$, which is impossible at least when $p=2$, as seen a few lines above. Similarly, as the optimality of $m$ in (1.29) is expected when $p>2$, the optimality of (1.32)-(1.33) in the case $p>2$ is expected too. In fact, this is the same argument used to get the optimality of Theorem 1.2 at the beginning of the section.

\subsection{Lebesgue vs Morrey}

Assuming (1.27) improves on (1.10) up to (1.29). Now assume that $\mu \in L^{t}$ for $t \in\left[1,\left(p^{*}\right)^{\prime}\right)$; in this case $D u \in L^{g}$ with $g=n t(p-1) /(n-t)[9,42]$; in particular $D u \in \mathcal{M}^{g}$. On the other hand $\mu \in L^{t}$ implies that $\mu$ satisfies (1.27) with $\theta=n / t$; in this case Theorem 1.8 gives $D u \in \mathcal{M}^{m}$ with $m=n(p-1) /(n-t)$, that is worse than $D u \in \mathcal{M}^{g}$, but for $t=1$. This does not contradict the sharpness of (1.29). Indeed we may find functions $f \in L^{1, \theta}$, with $\theta$ arbitrarily close to zero, such that $f \notin L^{t}$ for any $t>1$, see [34], comments at Chapter 2. On the other hand, truncation techniques fully apply in the case of $L^{t}$ data [45], because functions can be truncated, while measures cannot, and better integrability of $D u$ follows.

\subsection{Systems}

Theorem 1.10 extends to systems, under assumptions (1.2) and (1.4), when obviously recast for the vectorial case; $u: \Omega \rightarrow \mathbb{R}^{N}, z \in \mathbb{R}^{N \times n}$ and so on. In this case the measure $\mu$ takes its values in $\mathbb{R}^{N}$. Indeed for Theorem 1.10 we do not need Lemma 4.1; this employs the truncation operators (4.7) and they do not work for general elliptic systems. We also do not need Lemma 3.3, which under the general assumptions (1.2) only works in the scalar case. The only basic ingredients are Lemmas 4.4-4.5 and 3.2. The first two only need monotonicity in $(1.2)_{1}$, while the third one is here stated directly in the vectorial case $N \geq 1$. Anyway, we are planning further extensions to certain special classes of systems. 


\subsection{Condition (1.27)}

This can be relaxed in a local one, since the results we are giving are local. More precisely, we may assume that for every $\Omega^{\prime \prime} \subset \subset \Omega$ there exists a constant $M\left(\Omega^{\prime \prime}\right)$ such that

$$
|\mu|\left(B_{R}\right) \leq M\left(\Omega^{\prime \prime}\right) R^{n-\theta}, \quad \text { for every ball } B_{R} \subset \subset \Omega^{\prime \prime} .
$$

Roughly, we are considering $\mu \in L_{\text {loc }}^{1, \theta}(\Omega)$ rather than $\mu \in L^{1, \theta}(\Omega)$. When assuming (11.4) instead of (1.27) the inclusions of Theorems 1.8-1.12 still hold, but the a priori estimates change. We give the new statement for the estimate of Theorem 1.8 , the others to be modified in a similar fashion. For every couple of open subsets $\Omega^{\prime} \subset \subset \Omega^{\prime \prime} \subset \subset \Omega$ there exists a constant $c$ depending on $n, p, L / \nu, \Omega^{\prime}, \Omega^{\prime \prime}$

$$
\|D u\|_{\mathcal{M}^{m, \theta}\left(\Omega^{\prime}\right)} \leq c\|D u\|_{L^{p-1}\left(\Omega^{\prime \prime}\right)}+c\left[M\left(\Omega^{\prime \prime}\right)\right]^{1 /(p-1)}+c s\left|\Omega^{\prime \prime}\right|^{1 / m} .
$$

Moreover there exists $c$ depending on $n, p, L / v, \Omega^{\prime}, \Omega$ such that

$$
\|D u\|_{\mathcal{M}^{m, \theta}\left(\Omega^{\prime}\right)} \leq c\left[|\mu|\left(\Omega^{\prime \prime}\right)\right]^{1 /(p-1)}+c\left[M\left(\Omega^{\prime \prime}\right)\right]^{1 /(p-1)}+c s\left|\Omega^{\prime \prime}\right|^{1 / m} .
$$

\section{References}

[1] D. R. ADAMS, Traces of potentials arising from translation invariant operators, Ann. Scuola Norm. Sup. Pisa Cl. Sci. (3) 25 (1971), 203-217.

[2] D. R. AdAms, A note on Riesz potentials, Duke Math. J. 42 (1975), 765-778.

[3] D. R. Adams and L. I. HedberG, "Function Spaces and Potential Theory", Grundlehren der Mathematischen Wissenschaften, Vol. 314, Springer-Verlag, Berlin, 1996.

[4] R.A. AdAms, "Sobolev Spaces", Academic Press, New York, 1975.

[5] L. Ambrosio, N. Fusco and D. Pallara, "Functions of Bounded Variation and Free Discontinuity Problems", Oxford Mathematical Monographs. The Clarendon Press, Oxford University Press, New York, 2000.

[6] P. Benilan, L. Boccardo, T. Gallouët, R. Gariepy, M. Pierre and J. L. VÁzQUEZ, An $L^{1}$-theory of existence and uniqueness of solutions of nonlinear elliptic equations, Ann. Scuola Norm. Sup. Pisa Cl. Sci. (4) 22 (1995), 241-273.

[7] L. Boccardo, Problemi differenziali ellittici e parabolici con dati misure, Boll. Un. Mat. Ital. A (7) 11 (1997), 439-461.

[8] L. BocCARDo and T. GAllouËT, Nonlinear elliptic and parabolic equations involving measure data, J. Funct. Anal. 87 (1989), 149-169.

[9] L. BOCCARDO and T. GALlOUËT, Nonlinear elliptic equations with right-hand side measures, Comm. Partial Differential Equations 17 (1992), 641-655.

[10] L. BocCARDo, T. GAllouËT and L. ORsina, Existence and uniqueness of entropy solutions for nonlinear elliptic equations with measure data, Ann. Inst. H. Poincarè Anal. Non Linéaire 13 (1996), 539-551.

[11] B. BOJARSKI and T. IWANIEC, Analytical foundations of the theory of quasiconformal mappings in $\mathbb{R}^{n}$ Ann. Acad. Sci. Fenn. Ser. A I Math. 8 (1983), 257-324.

[12] L. CAfFARelli, Elliptic second order equations, Rend. Sem. Mat. Fis. Milano 58 (1988), 253-284. 
[13] L. CAFFARELLI, Interior a priori estimates for solutions of fully nonlinear equations, Ann. of Math. 126 (2) 130 (1989), 189-213.

[14] L. CafFarelli and I. Peral, On $W^{1, p}$ estimates for elliptic equations in divergence form, Comm. Pure Appl. Math. 51 (1998), 1-21.

[15] S. Campanato, Proprietà di inclusione per spazi di Morrey, Ricerche Mat. 12 (1963), $67-86$.

[16] S. Campanato, Proprietà di una famiglia di spazi funzionali, Ann. Scuola Norm. Sup. Pisa Cl. Sci. (3) 18 (1964), 137-160.

[17] S. Campanato, Equazioni elittiche non variazionali a coefficienti continui, Ann. Mat. Pura Appl. (4) 86 (1970), 125-154.

[18] S. CAMPANATO, Hölder continuity of the solutions of some nonlinear elliptic systems Adv. Math. 48 (1983), 16-43.

[19] G. R. CIRMI and S. LEONARDI, Regularity results for the gradient of solutions to linear elliptic equations with $L^{1, \lambda}$ data, Ann. Mat. Pura e Appl. (4) 185 (2006), 537-553.

[20] A. Dall' Aglio, Approximated solutions of equations with $L^{1}$-data. Application to the $H$-convergence of quasi-linear parabolic equations, Ann. Mat. Pura Appl. (4) 170 (1996), 207-240.

[21] G. Dal Maso, F. Murat, L. Orsina and A. Prignet, Renormalized solutions of elliptic equations with general measure data, Ann. Scuola Norm. Sup. Pisa Cl. Sci. (4) 28 (1999), 741-808.

[22] T. Del Vecchio, Nonlinear elliptic equations with measure data, Potential Anal. 4 (1995), 185-203.

[23] G. Di FAZIO and M. A. RAGUSA, Interior estimates in Morrey spaces for strong solutions to nondivergence form equations with discontinuous coefficients, J. Funct. Anal. 112 (1993), 241-256.

[24] G. Di Fazio, M. A. Ragusa and D. K. Palagachev, Global Morrey regularity of strong solutions to the Dirichlet problem for elliptic equations with discontinuous coefficients, J. Funct. Anal. 166 (1999), 179-196.

[25] M. Di Giampaolo and F. LeOnetTi, Boundedness of weak solutions to some linear elliptic equations with measure data, Differential Integral Equations 18 (2005), 1371-1382.

[26] G. Dolzmann, N. HungerbüHler and S. MÜller, The p-harmonic system with measure-valued right-hand side, Ann. Inst. H. Poincarè Anal. Non Linèaire 14 (1997), 353364.

[27] G. Dolzmann, N. HungerbüHLeR and S. MÜLLER, Uniqueness and maximal regularity for nonlinear elliptic systems of $n$-Laplace type with measure valued right-hand side, J. Reine Angew. Math. (Crelles J.) 520 (2000), 1-35.

[28] L. D'Onofrio and T. Iwaniec, Notes on p-harmonic analysis, Contemp. Math. 370 (2005), 25-49.

[29] L. Esposito, F. LeOnetTi and G. Mingione, Regularity results for minimizers of irregular integrals with $(p, q)$ growth, Forum Math. 14 (2002), 245-272.

[30] L. Esposito, F. Leonetti and G. Mingione, Sharp regularity for functionals with $(p, q)$ growth, J. Differential Equations 204 (2004), 5-55.

[31] V. FERONE and N. FUsCO, VMO solutions of the N-Laplacian with measure data, C. R. Acad. Sci. Paris Sèr. I Math. 325 (1997), 365-370.

[32] M. FuChS and J. ReUling, Non-linear elliptic systems involving measure data, Rend. Mat. Appl. (7) 15 (1995), 311-319.

[33] D. Gilbarg and N. S. Trudinger, "Elliptic Partial Differential Equations of Second Order", Grundlehren der Mathematischen Wissenschaften, Vol. 224. Springer-Verlag, BerlinNew York, 1977; second edition: 1998.

[34] E. Giusti, "Direct Methods in the Calculus of Variations", World Scientific Publishing Co., Inc., River Edge, NJ, 2003.

[35] L. Greco, T. IWANIEC and C. Sbordone, Inverting the p-harmonic operator, Manuscripta Math. 92 (1997), 249-258. 
[36] C. HAMBURger, Regularity of differential forms minimizing degenerate elliptic functionals, J. Reine Angew. Math. (Crelles J.) 431 (1992), 7-64.

[37] J. Heinonen, T. KilpeläInen and O. Martio, "Nonlinear Potential Theory of Degenerate Elliptic Equations", Oxford Mathematical Monographs., New York, 1993.

[38] T. IwANIEC, The Gehring lemma, In: "Quasiconformal mappings and analysis" (Ann Arbor, MI, 1995), 181-204, Springer, New York, 1998.

[39] T. IwANiEC and C. SBordone, Quasiharmonic fields, Ann. Inst. H. Poincaré Anal. Non Linèaire 18 (2001), 519-572.

[40] F. John and L. Nirenberg, On functions of bounded mean oscillation, Comm. Pure Appl. Math. 14 (1961), 415-426.

[41] T. KILPELÄINEN, Hölder continuity of solutions to quasilinear elliptic equations involving measures, Potential Anal. 3 (1994), 265-272.

[42] T. KilpeläInEN and G. LI, Estimates for p-Poisson equations, Differential Integral Equations 13 (2000), 791-800.

[43] T. KilpeläInen and J. MalÝ, The Wiener test and potential estimates for quasilinear elliptic equations, Acta Math. 172 (1994), 137-161.

[44] T. KilpeläInen, N. Shanmugalingam and X. ZHOng, Maximal regularity via reverse Hölder inequalities for elliptic systems of $n$-Laplace type involving measures, Preprint 2006.

[45] T. KilpeläInEN and XiangSheng XU, On the uniqueness problem for quasilinear elliptic equations involving measures Rev. Mat. Iberoamericana 12 (1996), 461-475.

[46] T. KILPELÄINEN and X. ZHONG, Removable sets for continuous solutions of quasilinear elliptic equations, Proc. Amer. Math. Soc. 130 (2002), 1681-1688.

[47] H. Kozono and M. YAmaZAKI, Semilinear heat equations and the Navier-Stokes equation with distributions in new function spaces as initial data, Comm. Partial Differential Equations 19 (1994), 959-1014.

[48] J. KRistensen and G. Mingione, The singular set of minima of integral functionals, Arch. Ration. Mech. Anal. 180 (2006), 331-398.

[49] G. M. Lieberman, Sharp forms of estimates for subsolutions and supersolutions of quasilinear elliptic equations involving measures, Comm. Partial Differential Equations 18 (1993), 1191-1212.

[50] G. M. Lieberman, A mostly elementary proof of Morrey space estimates for elliptic and parabolic equations with VMO coefficients, J. Funct. Anal. 201 (2003), 457-479.

[51] P. Lindevist, On the definition and properties of p-superharmonic functions, J. Reine Angew. Math. (Crelles J.) 365 (1986), 67-79.

[52] P. LiNDQVIST, "Notes on $p$-Laplace Equation", University of Jyväskylä - Lectures notes, 2006.

[53] J. L. Lions, "Quelques Méthodes de Résolution des Problèmes aux Limites non Linèaires", Dunod, Gauthier-Villars, Paris, 1969.

[54] W. Littman, G. Stampacchia and H. F. Weinberger, Regular points for elliptic equations with discontinuous coefficients, Ann. Scu. Norm. Sup. Pisa Cl. Sci. (3) 17 (1963), 43-77.

[55] J. MALÝ and W.P. ZIEMER, "Fine regularity of solutions of elliptic partial differential equations", Mathematical Surveys and Monographs, Vol. 51. American Mathematical Society, Providence, RI, 1997.

[56] J. J. MANFREDI, Regularity for minima of functionals with p-growth, J. Differential Equations 76 (1988), 203-212.

[57] J. J. MANFredi, "Regularity of the Gradient for a Class of Nonlinear Possibly Degenerate Elliptic Equations", Ph.D. Thesis, University of Washington, St. Louis.

[58] A. L. MazzUCATO, Besov-Morrey spaces: function space theory and applications to nonlinear PDE, Trans. Amer. Math. Soc. 355 (2003), 1297-1364.

[59] G. Mingione, The singular set of solutions to non-differentiable elliptic systems, Arch. Ration. Mech. Anal. 166 (2003), 287-301. 
[60] G. Mingione, Calderón-Zygmund estimates for measure data problems, C. R. Acad. Sci. Paris Sèr. I Math. 344 (2007), 437-442.

[61] G. MingIone, Sub-quadratic measure data problems, in preparation.

[62] T. MiYAKaWA, On Morrey spaces of measures: basic properties and potential estimates, Hiroshima Math. J. 20 (1990), 213-222.

[63] J. M. RAKOTOSON, Uniqueness of renormalized solutions in a $T$-set for the $L^{1}$-data problem and the link between various formulations, Indiana Univ. Math. J. 43 (1994), 685-702.

[64] J. Ross, A Morrey-Nikolski inequality, Proc. Amer. Math. Soc. 78 (1980), 97-102.

[65] T. RUNST and W. SicKEL, "Sobolev Spaces of Fractional Order, Nemytskij Operators, and Nonlinear Partial Differential Equations", Walter de Gruyter \& Co., Berlin, 1996.

[66] D. Sarason, Functions of vanishing mean oscillation, Trans. Amer. Math. Soc. 207 (1975), 391-405.

[67] J. SERRIN, Pathological solutions of elliptic differential equations, Ann. Scuola Norm. Sup. Pisa Cl. Sci. (3) 18 (1964), 385-387.

[68] G. STAMPACCHIA, Le problème de Dirichlet pour les équations elliptiques du second ordre à coefficients discontinus, Ann. Inst. Fourier 15 (1965), 189-258.

[69] G. Stampacchia, The spaces $\mathcal{L}^{(p, \lambda)}, N^{(p, \lambda)}$ and interpolation, Ann. Scuola Norm. Sup. Pisa Cl. Sci. (3) 19 (1965), 443-462.

[70] G. TALENTI, Nonlinear elliptic equations, rearrangements of functions and Orlicz spaces, Ann. Mat. Pura Appl. (4) 120 (1979), 160-184.

[71] M. E. TAYLOR, Analysis on Morrey spaces and applications to Navier-Stokes and other evolution equations, Comm. Partial Differential Equations 17 (1992), 1407-1456.

[72] K. UhlenBeCK, Regularity for a class of non-linear elliptic systems, Acta Math. 138 (1977), 219-240.

[73] X. ZHONG, On nonhomogeneous quasilinear elliptic equations, Dissertation, University of Jyväskylä, 1998, Ann. Acad. Sci. Fenn. Math. Diss. 117 (1998), 46 pages.

Dipartimento di Matematica Università di Parma

Viale G. P. Usberti 53/a, Campus 43100 Parma, Italy giuseppe.mingione@unipr.it. 